Florida International University FIU Digital Commons

$7-2-2013$

\title{
Differences Among Undergraduate and Graduate Nursing Students' Cultural Competency
}

Paula E. Seidel Glass

Florida International University, paulaglassnp@gmail.com

DOI: $10.25148 /$ etd.FI13080508

Follow this and additional works at: https://digitalcommons.fiu.edu/etd

Part of the Other Nursing Commons

\section{Recommended Citation}

Seidel Glass, Paula E., "Differences Among Undergraduate and Graduate Nursing Students' Cultural Competency" (2013). FIU

Electronic Theses and Dissertations. 940.

https://digitalcommons.fiu.edu/etd/940

This work is brought to you for free and open access by the University Graduate School at FIU Digital Commons. It has been accepted for inclusion in FIU Electronic Theses and Dissertations by an authorized administrator of FIU Digital Commons. For more information, please contact dcc@fiu.edu. 


\section{FLORIDA INTERNATIONAL UNIVERSITY \\ Miami, Florida}

\section{DIFFERENCES AMONG UNDERGRADUATE AND GRADUATE NURSING STUDENTS' CULTURAL COMPETENCY}

A dissertation submitted in partial fulfillment of the

requirements for the degree of

DOCTOR OF PHILOSOPHY

in

NURSING

by

Paula Ellen Seidel Glass 
To: Dean Ora Lea Strickland

College of Nursing and Health Sciences

This dissertation, written by Paula Ellen Seidel Glass, and entitled Differences Among Undergraduate and Graduate Nursing Students' Cultural Competency, having been approved in respect to style and intellectual content, is referred to you for judgment.

We have read this dissertation and recommend that it be approved.

Eric Fenkl

Paulette Johnson

Anahid Kulwicki

Date of Defense: July 2, 2013

Luz Porter, Major Professor

The dissertation of Paula Ellen Seidel Glass is approved.

Dean Ora Lea Strickland

College of Nursing and Health Sciences

Dean Lakshmi N. Reddi

University Graduate School

Florida International University, 2013 
(C) Copyright 2013 by Paula Ellen Seidel Glass

All rights reserved. 


\section{DEDICATION}

I dedicate this dissertation to my family. Without their love, patience, and support, the completion of this dissertation would not have been possible. 


\section{ACKNOWLEDGMENTS}

The completion of dissertation and doctoral degree has been one of most difficult tasks I have undertaken in my lifetime. This study could not have been completed without the support, guidance, and mentoring from the team of faculty and staff at Florida International University; Most notably, Dr. Luz Porter, Dr. Paulette Johnson, Dr. Eric Fenkl, and Dr. Anahid Kulwicki, thank you again. Special thanks goes to Dr. Stephanie Schim who granted me permission to use her survey for this study. Additional thanks to Dr. Schim for meeting at the Transcultural Nursing Society, Las Vegas, 2011, to discuss theoretical concepts.

Thank you to my daughters Jessica Alexis and Rebecca Lauren for your patience and understanding, having sacrificed family time, or shopping over the past five years. Thank you for the support throughout this educational endeavor. My daughters, both of you have said you are proud of me, your Mom, however, I am more proud of you. I am most grateful to my husband, Michael David who undertook unwanted domestic duties, grocery shopping, loss of "relax" time, walked Chloe and Riley, and helped in many ways while I earned my PhD in Nursing.

Lastly, my gratitude goes to all of the nursing students who supported this research by completing the surveys, necessary to complete this research and dissertation, thank you. 
ABSTRACT OF THE DISSERTATION

\title{
DIFFERENCES AMONG UNDERGRADUATE AND GRADUATE NURSING STUDENTS' CULTURAL COMPETENCY
}

\author{
by \\ Paula Ellen Seidel Glass \\ Florida International University, 2013 \\ Miami, Florida \\ Professor Luz Porter, Major Professor
}

This study examined differences in cultural competency levels between undergraduate and graduate nursing students (age, ethnicity, gender, language at home, education level, program standing, program track, diversity encounters, and previous diversity training). Participants were $83 \%$ women, aged 20 to $62 ; 50 \%$ Hispanic/Latino; with a Bachelor of Science in Nursing $(n=82)$ and a Master of Science in Nursing $(n=62)$. Degrees included high school diplomas, associate/diplomas, bachelors' degrees in or out of nursing, and medical doctorate degrees from outside the United States. Students spoke English $(n=82)$ or Spanish $(n=54)$. The study used a cross-sectional design guided by the three-dimensional cultural competency model. The Cultural Competency Assessment (CCA) tool is composed of two subscales: Cultural Awareness and Sensitivity (CAS) and Culturally Competent Behaviors (CCB). Multiple regressions, Pearson's correlations, and ANOVAs determined relationships and differences among undergraduate and graduate students. Findings showed significant differences between undergraduate and graduate nursing students in CAS, $p<.016$. Students of Hispanic/White/European ethnicity scored higher on the CAS, while White/non-Hispanic students scored lower on the CAS, $p<.05$. 
One-way ANOVAs revealed cultural competency differences by program standing (grade-point averages), and by program tracks, between Master of Science in Nursing Advanced Registered Nurse Practitioners and both Traditional Bachelor of Science in Nursing and Registered Nurse-Bachelor of Science in Nursing. Univariate analysis revealed that higher cultural competency was associated with having previous diversity training and participation in diversity training as continuing education. After controlling for all predictors, multiple regression analysis found program level, program standing, and diversity training explained a significant amount of variance in overall cultural competency $\left(p=.027 ; R^{2}=.18\right)$. Continuing education is crucial in achieving students' cultural competency. Previous diversity training, graduate education, and higher gradepoint average were correlated with higher cultural competency levels. However, increased diversity encounters were not associated with higher cultural competency levels. 


\section{TABLE OF CONTENTS}

CHAPTER

PAGE

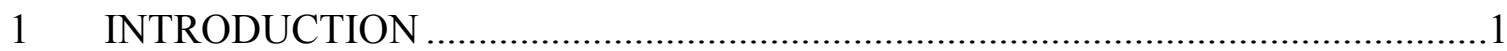

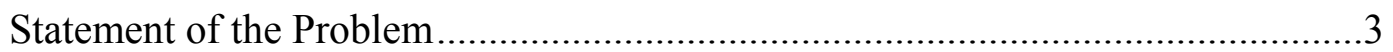

Purpose of the Study ………......................................................................

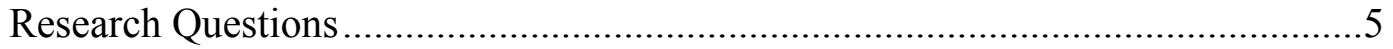

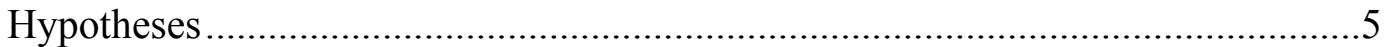

Conceptual Definitions .........................................................................................

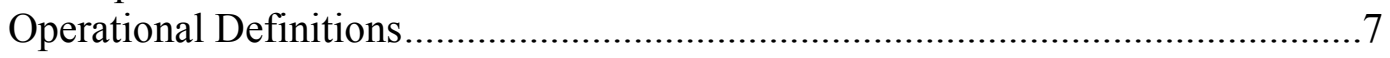

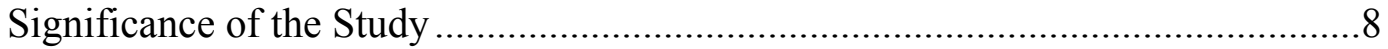

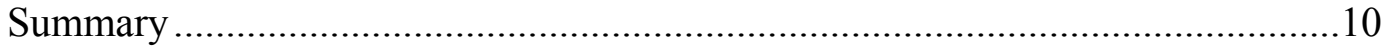

2 LITERATURE REVIEW …………………………......................................

Theoretical Frameworks and Models of Cultural Competency in Related

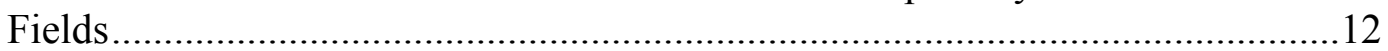

Taxonomy of Educational Objectives (Bloom, 1956) ………………………....12

Health-belief model (Rosenstoch, 1974) ...........................................................12

Patients' explanatory model (Kleinman, 1978) ..................................................13

The LEARN, ADHERE, and ETHNIC models (Berlin \& Fwokes, 1983;

Levin, Like, \& Gottlieb, 2000; Soto-Greene, Sanchez, Salar-Lopez, \& Like,

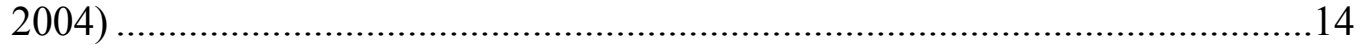

Experiential theory $($ Kolb, 1984) ..................................................................

Cultural competency Theories and Models Developed by Nurse Theorists...........16

Theory of culture-care diversity and universality (Leininger, 1988)....................16

Cultural-safety model (Ramsden \& Spoonley, 1994)..........................................17

The Rew pathway model $($ Rew, 1996) …………………..................................17

Cultural competence and confidence model (Jeffreys, 2000)..............................18

Cultural-development model (Wells, 2000) ......................................................18

Matrix for growth through transcultural immersion (Ryan \& Twibell, 2002) ...19

Cultural Competence in Healthcare Scale (Caffrey, Neander, Markle, \&

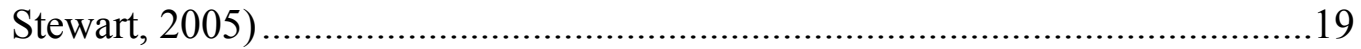

Model of cultural competence (Purnell, 2005) .................................................20

The process of cultural competence in the delivery of healthcare model

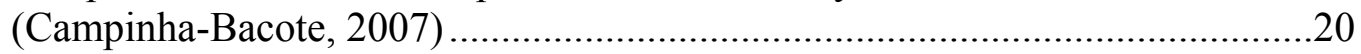

Transcultural-assessment model (Andrews \& Boyle, 2008) ..............................21

Cultural competency-development model (Papadopoulos, Tilki, \& Taylor,

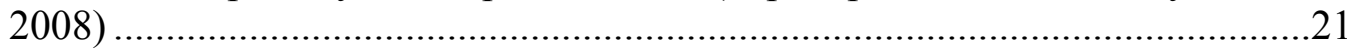

The health-traditions model (Spector, 2009) …………………………….........22

Culture-competency model (CCM; Schim \& Doorenbos, 2010) ……...............22

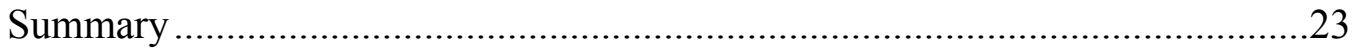

Research on Cultural Competency................................................................24

Cultural Competency Research in Nursing Education ......................................28 
Research on the Cultural competency Model (CCM) and Cultural competency

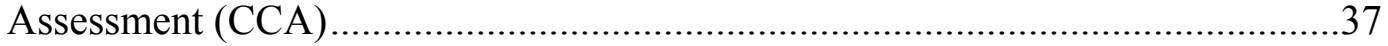

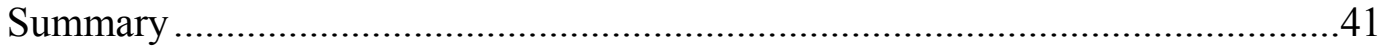

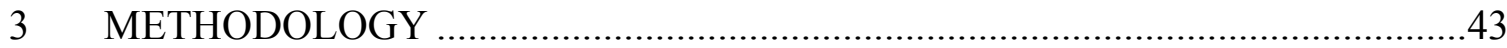

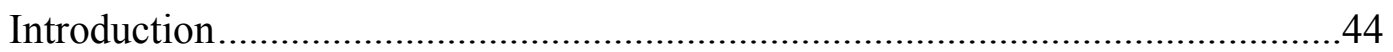

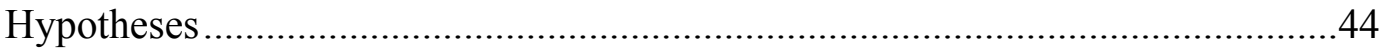

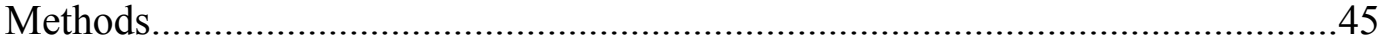

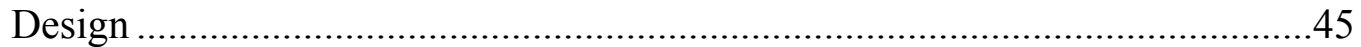

Sample

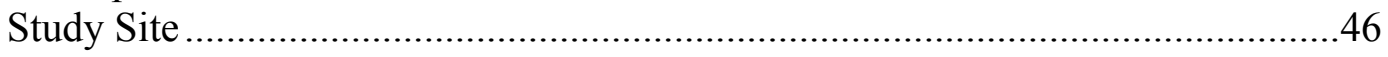

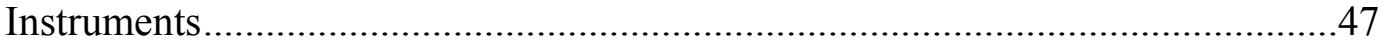

The Cultural Competency Assessment Scale ……………………………….....47

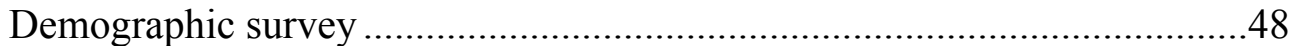

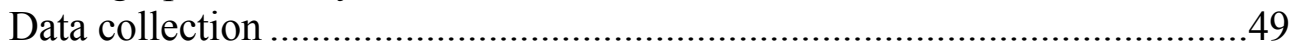

Psychometric evaluation of the cultural competency assessment..................50

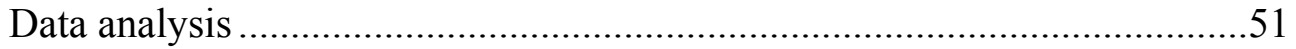

Independent and dependent variables summarized.....................................52

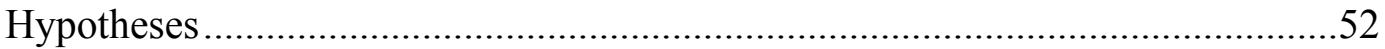

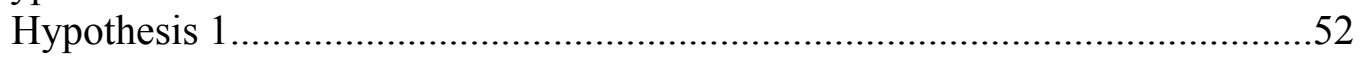

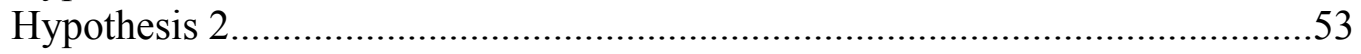

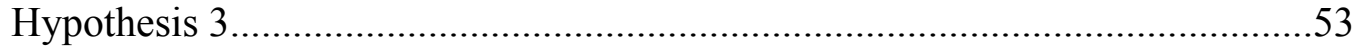

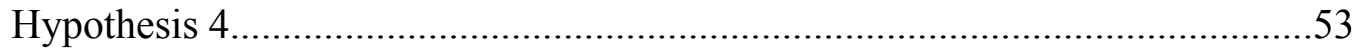

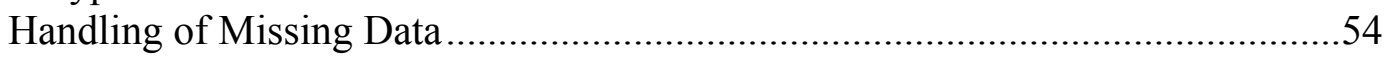

Human Subjects and Inclusion Criteria …………….......................................54

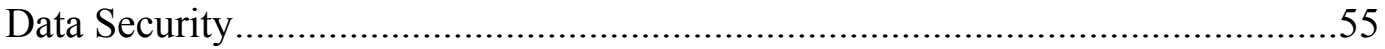

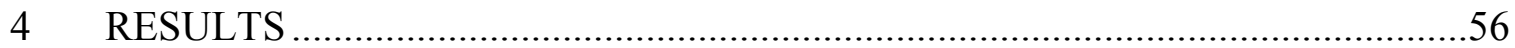

Data Screening and Sample Size …………………...................................5

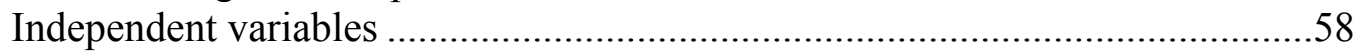

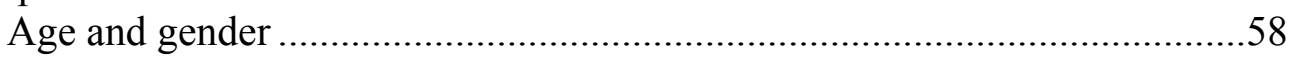

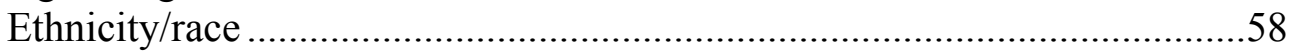

Language spoken at home.........................................................................6

Degree level attained, educational level enrolled, program track and

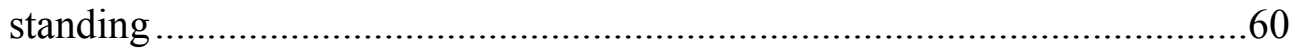

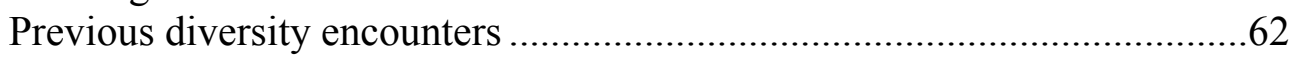

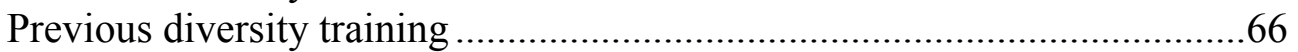

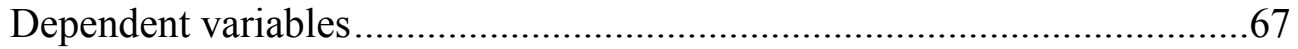

Hypotheses and Findings .....................................................................................

Hypothesis 1: Levels of nursing education and cultural competency..................71

Hypothesis 2: Cultural competency levels and program standing (GPA) ...........74

Hypotheses 3 and 4: Personal and social factors and cultural competency .........75

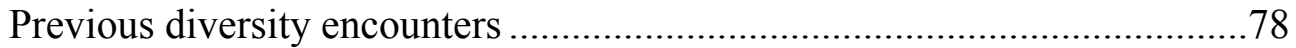

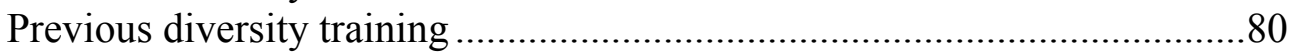

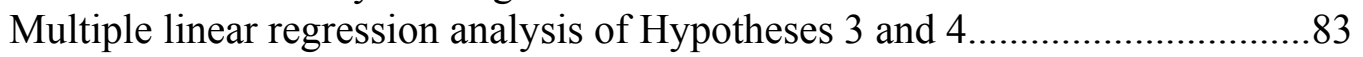




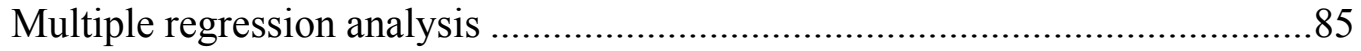

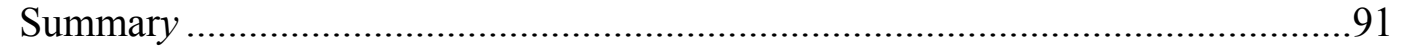

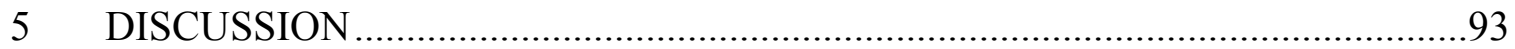

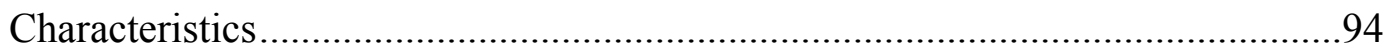

Educational Factors, Differences Between Nursing-Student Groups ....................95

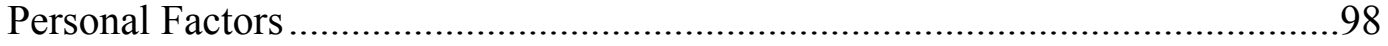

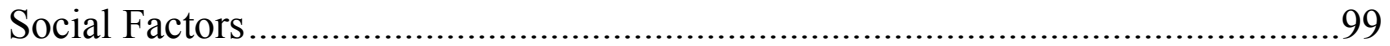

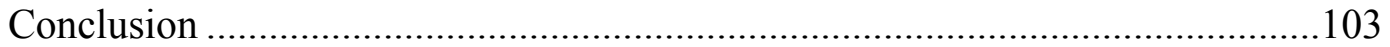

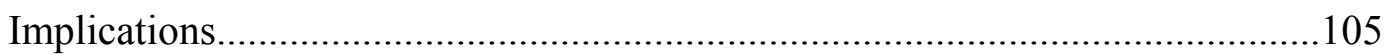

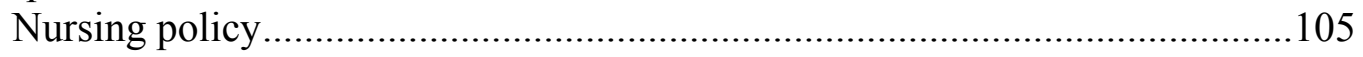

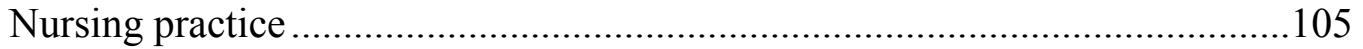

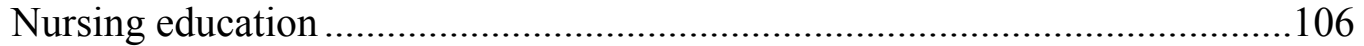

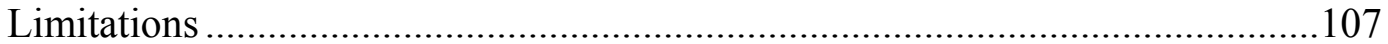

Recommendations for Future Research ....................................................10

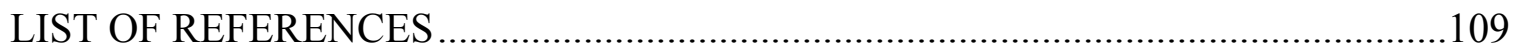

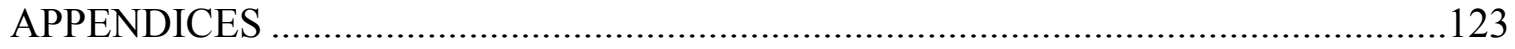

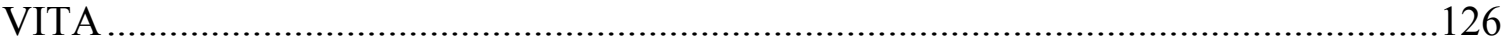




\section{LIST OF TABLES}

TABLE

PAGE

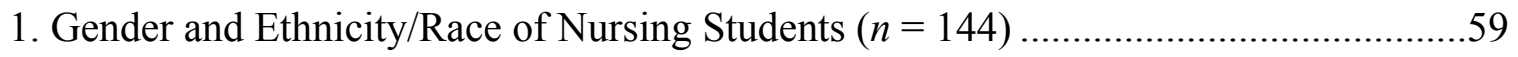

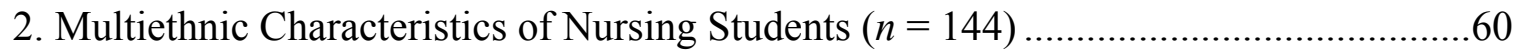

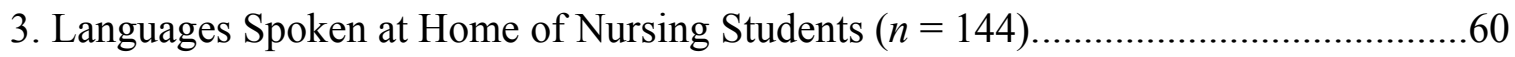

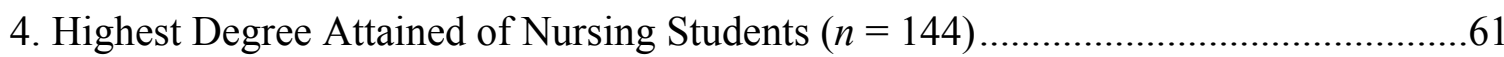

5. Educational Level, Program track, and Program Standing (GPA) of Nursing

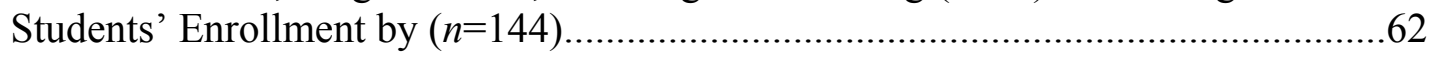

6. Diverse Ethnicities/Races Encountered in Nursing Students' Environments with

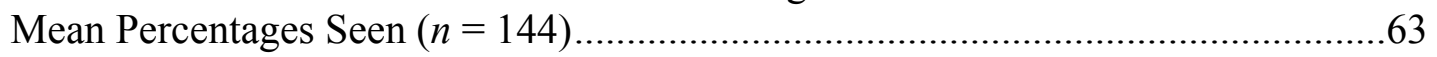

7. Number of Ethnic/Racial Groups Encountered in Nursing Students' Environments

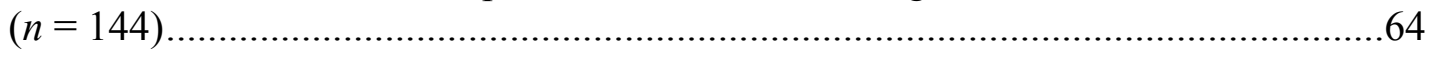

8. Diverse Special Populations Encountered in Nursing Students' Environments $(n=144)$.

9. Number of Special Populations Encountered in Nursing Students' Environments (Maximum of $7 ; n=144$ ).

10. Previous Diversity Training and Types of Diversity Training Nursing Students Attended $(n=144)$

11. Number of Types of Previous Diversity Training Attended by Nursing Students .......

12. Cultural Awareness and Sensitivity Subscale Characteristics of Nursing Students $(n=144)$

13. Cultural Competent Behaviors Subscale Characteristics of Nursing Students $(n=$ 140) .70

14. Total Cultural Competency Assessment, Cultural Awareness and Sensitivity, and Cultural Competent Behaviors Reliabilities .70

15. Self-Evaluated Cultural Competency Levels of Nursing Students $(n=144)$ .71

16. Means of Total Cultural Competency Assessment Scale, Cultural Awareness and Sensitivity, and Cultural Competent Behaviors Subscales by Program level 
17. Means of Total Cultural Competency Assessment, Cultural Awareness and Sensitivity, and Cultural Competent Behaviors Subscales by Program track .73

18. Cultural Competency of Nursing Students by Program Standing .74

19. Correlations of Total Cultural Competency Assessment, Cultural Awareness and Sensitivity, and Cultural Competent Behaviors Subscale Scores and Student Age ..76

20. Total Cultural Competency Assessment, Cultural Awareness and Sensitivity, and Cultural Competent Behaviors By Gender

21. Cultural Competency Assessment, Cultural Awareness and Sensitivity, and Cultural Competent Behaviors by Ethnicity/Race.

22. Cultural Competency Assessment, Cultural Awareness and Sensitivity, and Cultural Competent Behaviors by Language

23. Pearson's Correlations of Total Cultural Competency Assessment, Cultural Awareness and Sensitivity, and Cultural Competent Behaviors Subscale Scores with Number of Ethnic Groups Encountered and Each Type of Ethnic Group Encountered

24. Pearson's Correlations of Total Cultural Competency Assessment, Cultural Awareness and Sensitivity, and Cultural Competent Behaviors Subscales with Number of Special Populations Encountered and Each Special Population Encountered

25. Total Cultural Competency Assessment, Cultural Awareness and Sensitivity, and Cultural Competent Behaviors Subscale Scores by Previous Diversity Training .....81

26. Pearson's Correlations of Total Cultural Competency Assessment, Cultural Awareness and Sensitivity, and Cultural Competent Behaviors Subscale Scores with Diversity Training Measures

27. Pearson's Correlations of Total Cultural Competency Assessment, Cultural Awareness and Sensitivity, and Cultural Competent Behaviors Subscale Scores with Personal Factors

28. Pearson's Correlations of Total Cultural Competency Assessment, Cultural Awareness and Sensitivity, and Cultural Competent Behaviors Subscale Scores with Education, Diversity Training, and Encounters

29. Model Summary of Regression of Total Cultural Competency Assessment on Personal, Educational, Diversity Training, and Diversities Encountered.

30. Model Summary of Regression of Cultural Awareness and Sensitivity Subscale on Personal, Educational, Diversity Training, and Diversities Encountered 
31. Model Summary of Regression of Cultural Competent Behaviors Subscale on Personal, Educational, Diversity Training, and Diversities Encountered....................90 


\section{ACRONYMS AND ABBREVIATIONS}

\begin{tabular}{|c|c|}
\hline $\mathrm{AACN}$ & American Association of Colleges of Nursing \\
\hline ARNP & Advanced Registered Nurse Practitioner \\
\hline BSN & Bachelor of Science in Nursing \\
\hline CAS & Cultural Awareness and Sensitivity \\
\hline CCA & Cultural Competency Assessment \\
\hline $\mathrm{CCB}$ & Culturally competent behavior \\
\hline $\mathrm{CCM}$ & Cultural competency model \\
\hline CDM & Cultural-development model \\
\hline CSES & Cultural Self-Efficacy Scale \\
\hline EM & Patient's explanatory model \\
\hline FEP & Foreign-educated physician \\
\hline GPA & Grade-point average \\
\hline HBM & Health-belief model \\
\hline $\mathrm{IAPCC}^{\circledR}$ & $\begin{array}{l}\text { Inventory for Assessing the Process of Cultural Competence among } \\
\text { Healthcare Professionals }\end{array}$ \\
\hline IRB & Institutional Review Board \\
\hline MSN & Master of Science in Nursing \\
\hline NLN & National League for Nursing \\
\hline NP & Nurse practitioner \\
\hline $\mathrm{OMH}$ & $\begin{array}{l}\text { U.S. Department of Health and Human Services, Office of Minority } \\
\text { Health }\end{array}$ \\
\hline
\end{tabular}




\section{Chapter 1}

Introduction 


\section{CHAPTER 1}

\section{INTRODUCTION}

A continuing increase in U.S. population diversity has produced societal challenges for healthcare providers and healthcare systems (Douglas \& Pacquiao, 2010; Hoeffer, Rytina, \& Baker, 2010; Institute of Medicine, 2003; Smedley \& Stith, 2002). Effective cross-cultural communication enhances provider-client relationships, greater patient satisfaction, and adherence to treatment (Gaskin \& Hoffman, 2000; Munoz \& Luckmann, 2005; U.S. Department of Health and Human Services, Office of Minority Health $[\mathrm{OMH}], 2001)$. Experts in transcultural nursing (Douglas \& Pacquiao, 2010) asserted that general cultural knowledge and awareness approaches ensure that healthcare providers ask appropriate questions of patients from different backgrounds. Based on scientific data supporting culturally and linguistically appropriate healthcare services to improve patient-care outcomes (Gaskin \& Hoffman, 2000; OMH, 2001), federal and state governments, and accrediting agencies mandate that clinicians be culturally competent (Jenakovich et al., 2001; Joint Commission, 2010; Ryan, Carlton, \& Ali, 2000). However, cultural competency is a dynamic concept and process that makes knowledge about all diverse cultures impossible (Andrews \& Boyle, 2008; Cross, Bazron, Dennis, \& Isaacs, 1989; Schim \& Doorenbos, 2010). Although a plethora of resources exists, they have yielded confusion and a lack of agreement among nursing educators as to the amount and type of cultural-specific content and delivery methods that will produce culturally competent practitioners. 


\section{Statement of the Problem}

Researchers supported the incorporation of culturally competent content and learning experiences in the curricula for each health profession (American Association of Colleges of Nursing [AACN], 2008b; American Nurses Association, 1991; Betancourt, 2007; National League for Nursing [NLN], 2008). Multiple theories and frameworks have roots in each discipline, with their own lists of conceptual definitions (Douglas \& Pacquiao, 2010). Experts agreed that cultural competency has three components: a cultural-knowledge or cognitive domain, a cultural-awareness and sensitivity or affective domain, and a culturally competent behavioral domain. Students need to master all three domains to achieve various cultural competency levels (Campinha-Bacote, 2007; Galanti, 2008; Giger \& Davidhizar, 2008; Leininger \& McFarland, 2006; Purnell, 2005; Schim \& Doorenbos, 2010; Spector, 2009). Cultural awareness and sensitivity have been difficult to evaluate and continuing research is needed.

Incorporation of cultural content into nursing curricula presents many challenges. Factors to be considered in curriculum design should include students' educational levels, learning needs, cultural-content objectives, language barriers, and varying degrees of existing cultural competency levels. Nursing education and research reviewed did not specifically address the best combination of factors but often evaluated specific

educational interventions. Education strategies evaluated have been in clinical, classroom, and laboratory settings. Although clinical courses use local community or international experiences, those can be inconsistent. Recent research has not demonstrated where nursing students' cultural competency levels are today without the use of culturaleducational interventions. In addition, students' perceptions about overall curriculum at 
different levels and specialties at a public university with unique specialty tracks and a diverse student body have not been recently evaluated (Alpers \& Zoucha, 1996; Schim, Doorenbos, Benkert, \& Miller, 2007).

The individuality and combination of factors evaluated in different studies suggested the need for each institution to perform its own evaluation, prior to planning any improvements for cultural competency goals (Jeffreys, 2010). Therefore, each nursing program will need to evaluate cultural competency outcomes independently. Culturally competent behaviors are measurable and include cross-cultural communication that builds provider-client relationships, increases patient satisfaction and adherence to treatments, and promotes better outcomes (Cooper et al., 2009). Culturally competent behaviors include seeking cultural information, effective use of cross-cultural communication, interpreters, proper translation of patient or student materials, and documentation of culturally or otherwise diversely adapted care (Schim \& Doorenbos, 2010). Effective interactions ensure accurate patient data collection and that correct treatments are ordered (Andrews \& Boyle, 2008; Munoz \& Luckmann, 2005; Schim et al., 2007).

\section{Purpose of the Study}

The purpose of this study was to investigate differences in cultural competency among undergraduate and graduate nursing students attending a public institution. The study sought to determine if students' educational level, program standing (grade-point average; GPA), program track, and personal or social factors are associated with students' cultural competency levels. 


\section{Research Questions}

1. What are the differences in cultural competency levels between undergraduate (Registered nurse-Bachelor of Science in Nursing [RN-BSN] Online, foreigneducated physician (FEP) BSN, and Traditional BSN) and graduate nursing students (Adult, Child, Family Advanced Registered Nurse Practitioners [ARNPs], Anesthesiology, \& FEP BSN/Master of Science in Nursing $[\mathrm{MSN}]) ?$

2. To what extent are students' cultural competency levels associated with their academic standing (GPA)?

3. What personal factors (age, ethnicity/race, gender, language at home) or social factors (previous number of diversity encounters or previous diversity training) are associated with students' cultural competency levels?

\section{Hypotheses}

1. Higher levels of education are associated with higher cultural competency levels as measured by
a. cultural awareness and sensitivity (CAS subscale)
b. culturally competent behaviors (CCB subscale)
c. cultural competency assessment (CCA) (total scale mean)
d. self-evaluation of cultural competency (rated 1-5 on the Likert-style scale)

2. Higher program standing (GPA) of students is associated with higher cultural competency levels.

3. Students' cultural competency levels are not associated with the following personal factors: 


\section{1. age}

\section{2. gender}

3.3. ethnicity/race

3.4. language spoken at home

4. Students' cultural competency levels are associated with the following social factors:

4.1. greater number of diversity encounters over the past 12 months

4.2. previous diversity training

\section{Conceptual Definitions}

As used in this study, the key terms are defined as follows:

1. Culture is a product of any number of characteristics such as: age, race/ethnicity, gender, religious views, linguistics, education of students and parents, socioeconomic status, and housing security.

2. Cultural competency is a dynamic evolving process that leads to effective interactions between providers and patients of different backgrounds. The components of cultural competency in this model include cultural awareness and knowledge, cultural sensitivity, cultural diversity, and culturally congruent care (Schim \& Doorenbos, 2010).

3. Cultural awareness is the cognitive domain of the model and requires obtaining knowledge and insight about culturally diverse clients' heritages (Campinha-Bacote, 2007; Schim et al., 2007). Cultural desire is an assumption in the cultural competency process and cannot be taught. 
4. Cultural sensitivity refers to the affective or attitudinal domain of cultural competency in this model (Cooper et al., 2009; Schim et al., 2007). Sensitivity assumes self-reflection and awareness of personal ethnocentricities that may interfere with effective cross-cultural interactions (Campinha-Bacote, 2007; Schim et al., 2007).

5. Cultural diversity refers to any biological, personal, or social characteristics that vary from the dominant cultures and many subcultures. Cultural and otherwise diverse individuals exist in one's daily life and are a fact of life (Schim et al., 2007).

\section{Operational Definitions}

As used in this study, the key terms are operationalized as listed below:

1. Cultural competency is the sample mean score obtained from the total CCA scale.

2. Cultural awareness is the sample mean score obtained from the CAS subscale.

3. Cultural sensitivity is the sample mean score obtained from the CAS subscale.

4. Culturally competent behaviors is the sample mean score obtained from the CCB subscale, which measures behaviors such as seeking cultural information, effective use of cross-cultural communication and use of interpreters, as well as proper interpretation of students' and patients' materials.

5. Educational level refers to undergraduate or baccalaureate nursing students (FEP-BSN, RN-BSN Online, and Traditional BSN) and graduate nursing students (Adult, Child, and Family Advanced Registered Nurse Practitioners, Anesthesiology nursing, and FEP Accelerated BSN/MSN). 
6. Previous diversity encounters connote interaction of any type with various diverse cultural and special population groups at work or other settings over the past 12 months.

7. Previous diversity training refers to a gain in cultural-diversity knowledge or skills from various sources such as college courses for credit, cultural content in other courses, continuing education, employer-sponsored programs, or computed-assisted learning programs.

8. Male or female gender as self-reported by the respondent.

9. Ethnicity refers to the heritage, nationality group, lineage, or country of birth of a person or a person's parents or ancestors before their arrival in the United States.

10. Race denotes a grouping of populations on the basis of various sets of physical characteristics that result from genetic ancestry.

11. Language refers to a basic form of communication, spoken most frequently at home or in social interactions.

\section{Significance of the Study}

Experts agree that the mission of undergraduate and graduate nursing education is to prepare graduates for practice in the environments they will encounter (Ervin, Bickes, \& Schim, 2006). Cultural competency is an important aspect of practice as well as an accreditation requirement (Riley, 2010). Nursing programs need evidenced-based research to guide the incorporation of cultural competency into various curricula. This study involved undergraduate and graduate students enrolled in specialty tracks with unique backgrounds. It sought to determine significant associations among personal, 
demographic, and previous diversity exposure or training factors and cultural competency levels attained. The knowledge gained from this study may support valued changes for nursing education and future research.

This study is important for many stakeholders: academic institutions, nursing programs, students, parents, faculty, staff, and healthcare institutions. Universities and nursing programs must evaluate their students' cultural competency levels; and the study of the cultural competency of students from diverse backgrounds in various specialty tracks may offer significant knowledge. Of note, most research captures results only after a specific intervention, whereas this research evaluated undergraduate and graduate students' cultural competency levels at different points in their academic-program progression. This study offers insight to nursing educators, accredited institutions, students, and graduates serving in diverse communities.

Nursing students are as diverse as residents in communities. It behooves healthcare providers to be culturally and linguistically appropriate, thus the need to continually develop cultural competency in schools and through continuing education (Culturally Linguistic Appropriate Services, OMH, 2001; Joint Commission, 2010). The culturally diverse students of today have many characteristics developed outside of academic curricula and personal or professional experiences that can significantly influence or contribute to the cultural competency levels they attain. This research has shed more light on the impact of personal and social factors that can contribute to or inhibit the development of cultural competency. 


\section{Summary}

Research has shown evidence that providers' with culturally competent practice skills offer effective culturally congruent care. The challenge arises when students' cultural knowledge and skills are not appropriately evaluated for effectiveness of cultural competency levels attained, despite these factors being taught in academic programs and throughout the curriculum. This research is potentially important to transcultural experts, researchers, and nursing educators in undergraduate and graduate nursing programs.

Knowledge gained from this study should be considered in the development of evidencebased, culturally competent curriculum. 


\section{Chapter 2}

\section{Literature Review}




\section{CHAPTER 2}

\section{LITERATURE REVIEW}

This chapter presents a review of the literature on different theoretical frameworks and models addressing cultural competency, as well as research studies conducted on cultural competency in nursing education. The review includes instruments used to measure the cultural competency of students and healthcare providers, and a comprehensive review of the three-dimensional cultural competency model and the CCA tool developed by Schim and Doorenbos (2010). The chapter concludes with a summary.

\section{Theoretical Frameworks and Models of Cultural Competency in Related Fields}

Many transcultural theories, models, and concepts in nursing include concepts and propositions that are derived from other disciplines (Goode, Dunne, \& Bronheim, 2006). In 1982, Benner developed a theory of "novice to expert," built on the Dreyfus model, a theoretical model from another field. In Benner's theory $(1982,2001)$, students are assisted in their transition from new nurses or novices to experts possessing instinctual knowledge and skills. To become an expert in the field, the novice must go through the process of being an advanced beginner to becoming competent, then to proficient, and ultimately, to being an expert (Benner, 2001). These concepts have been applied and are supported to be quite useful in nursing education and research (Benner, 1982; CampbellHeider, Rejman, Austin-Ketch, Sackett, \& Feeley, 2006; Duke, Connor, \& McEldowney, 2009).

Taxonomy of Educational Objectives (Bloom, 1956). The Taxonomy of Educational Objectives, developed in 1956 by a group of educational psychologists headed by Bloom, is a classification of levels of intellectual behavior important in 
learning (Anderson \& Krathwohl, 2001). The original taxonomy constructs include knowledge, comprehension, application, analysis, synthesis, and evaluation (Abrums \& Leppa, 2001; Cuellar, Brennan, Vito, \& Siantz, 2008). Commonly known as Bloom's Taxonomy of Cognitive Levels, it has been often used by nursing programs to manage curricula and examinations (Abrums \& Leppa, 2001; Anderson \& Krathwohl, 2001; Campbell-Heider et al., 2006; Cuellar et al., 2008).

Health-belief model (Rosenstoch, 1974). Similarly, the health-belief model (HBM) was created to guide understanding of general and culture-specific concepts relevant to providing culturally competent and congruent care (Douglas et al., 2011; Fortier \& Bishop, 2003). The HBM was also created to explain correlations "between an individual's health perceptions and individual's health preventative behaviors" (Douglas \& Pacquiao, 2010, p. 86s). Theoretical constructs of the HBM include individuals' perceptions, modifying factors, and the likelihood of action (p. 87s). A concept in the HBM seen in other models was that self-efficacy plays an important role in individuals' belief in their ability to perform preventative actions (Bandura, 1977; Douglas \& Pacquiao, 2010, p. 88s).

Patient's explanatory model (Kleinman, 1978). The patient's explanatory model (EM), one of the earliest frameworks, was developed to help patients and physicians define and understand a patient's meanings and social connections of health and illness (Douglas \& Pacquiao, 2010). The EM helps explain culturally based illnesses (i.e., susto). A major concept of the EM includes the emic explanations of why an illness develops and how it should be treated (Douglas \& Pacquiao, 2010). The EM was used to 
create the explanatory, social, fears, and treatments model or ESFT mnemonic. (Betancourt, 2006).

The LEARN, ADHERE, and ETHNIC models (Berlin \& Fowkes, 1983; Levin, Like, \& Gottlieb, 2000; Soto-Greene, Sanchez, Salar-Lopez, \& Like, 2004). The LEARN, ADHERE, AND ETHNIC models are a few of several mnemonic tools created to improve patient-centered cultural and clinical assessments (Douglas \& Pacquiao, 2010). Each letter of the LEARN mnemonic identifies a step in the interview or assessment process. The "L" refers to listening with sympathy and understanding to the patient's perception of the problem. The "E" refers to one's explanation or perceptions of a problem. The "A" refers to acknowledgement and discussion of differences and similarities revealed. The " $\mathrm{R}$ " refers to recommend treatment, and the " $N$ " refers to negotiate an agreed on treatment regimen (Berlin \& Fowkes, 1983). The LEARN model was used concurrently with the three-dimensional cultural competency model to create and analyze the effectiveness of a cultural competency web-based continuing-education course (Doorenbos et al., 2010).

The ADHERE model (mnemonic) expanded on the EM for use also as a crosscultural interview tool (Soto-Greene et al., 2004). Each letter of the ADHERE mnemonic guides the interview process. The " $\mathrm{A}$ " refers to acknowledging the need for treatment with the patient, asking about previous treatments used, and determining mutual goals and desired outcomes (Soto-Greene et al., 2004, p.33). The "D" refers to discussing potential treatments, options, and consequences of no treatment (p.33). The " $\mathrm{H}$ " refers to handling a patient's questions or concerns about the treatment. The "E" represents evaluation of the patient's functional health literacy and understanding of the purpose and 
rationale for treatment, as well as assessing barriers and facilitators to treatment adherence (p. 33). The " $R$ " represents recommending treatment and reviewing the regimen with the patient. Lastly, the "E" refers to empowering a patient's commitment and willingness to follow the therapeutic regimen prescribed (Soto-Greene et al., 2004, p.33). Researchers used the ADHERE and ETHNIC models as interview frameworks with medical students (Soto-Greene et al., 2004).

The ETHNIC tool is also an interview guide with each letter referring to explanation, treatment, healers, negotiation, intervention, and collaboration (p. 32). Findings showed that both the ADHERE and ETHNIC mnemonics were successful at identifying barriers and risk factors for patients' adherence to treatment regimens (SotoGreene et al., 2004).

Experiential theory (Kolb, 1984). Kolb (1984) integrated the works of Dewey, Lewin, and Piaget to emphasize the importance of experiences in "the learning process" (p. 20). Experiential theory has guided learning objectives and planned experiences for clinical, community health, skills laboratories, Internet gaming, simulated learning, and local and international immersions in nursing and other disciplines (Assemi, Cullander \& Hudmon, 2004; Barton \& Brown, 1992; Carpio \& Majumdar, 1993; Graham \& Richardson, 2008; Hertel \& Millis, 2002; Kolb, 1984). Major concepts include experience, perception, cognition, and behavior (Kolb, 1984, p.21). Experiential learning strategies expose students to client-care situations that increase cultural awareness and competency, which may not be available in clinical settings. Role play has been used with undergraduate students to increase cultural awareness; however, this teaching strategy has not been adequately evaluated (Shearer \& Davidhizar, 2003). 


\section{Cultural competency Theories and Models Developed by Nurse Theorists Theory of culture-care diversity and universality (Leininger, 1988).}

Leininger's (1988) developed the theory of culture-care diversity and universality, first introduced as the sunrise model (Leininger, 1995). Leininger's work was seminal in the history of cultural competency models used in nursing-education programs and research, with ethnographic, qualitative, and documented immersion among individuals, families, and communities of diverse backgrounds. This theory supports the concept that cultural competency occurs on a continuum from wellness and disease prevention to eventual illness or loss of life. Leininger asserted that attaining cultural competency requires passing through phases of increased knowledge and application of skills.

The sunrise model is a complex schema shaped as a burst of sunrise depicting multiple levels and factors that influence culturally congruent care of individuals, families, and communities. The theory and model have helped formulate a vision and structure of culturally competent curricula across all levels of nursing education (Leininger, 1995). However, Leininger’s “curricular approach” would require transcultural nursing courses for degree completion and the "establishment and maintenance of transcultural institutes," because at that time, "less than 20 percent of faculty and less than two percent of doctoral students" were formally prepared in transcultural nursing (Leininger, 1995, p. 12). Recent uses of Leininger's theory to guide and evaluate curricula outcomes continue to be supported (Mixer, 2008; 2011). However, extensive work is involved in adopting this model as a curriculum framework (Mixer, 2011). 
Cultural-safety model (Ramsden \& Spoonley, 1994). The importance of cultural competence in healthcare delivery was of major interest in New Zealand. Ramsden and Spoonley (1994) developed the cultural safety model to enhance patient safety, increase awareness of the importance of effective cross-cultural communication, and recognize diversity of worldviews (Douglas \& Pacquia, 2010; Papps \& Ramsden, 1996). This model's significance to transcultural nursing is the creation of a healthcaresetting culture of patient safety that includes continuous quality improvements and reduction of healthcare disparities experienced by diverse, vulnerable populations (Papps \& Ramsden, 1996). Assumptions of this model included recognition of differences, provision of respectful care for individual differences, and, professional awareness of social and political forces that influence empowerment in health care and status for the Maori people (Douglas \& Pacquiao, 2010, p. 83s).

The Rew pathway model (Rew, 1996). This model was built on Bronfenbrenner's bioecological theory of human development (Rew, 1996). The purpose of the Rew pathway model was to enhance nursing education and faculty development by providing mentors for students from "disadvantaged backgrounds" (Rew, 1996, p. 310). The goal of the RPM was to promote effective student and faculty interactions and strategies to increase students' cultural-diversity adaptability (Rew, 1996). Goals of the model included increased self-perceived competency as a result of curriculum, faculty, and planned learning experiences. Presumably, attitudes and values being formed by interpersonal interactions and behavioral skills are developed through the collaboration of people and their environments. An important aspect of this model is that it was intended specifically for interactions between nursing mentors and students (Rew, 1996). 
Cultural competence and confidence model (Jeffreys, 2000). Jeffreys (2000) developed the cultural competency and confidence model, applying self-efficacy or confidence concepts from Bandura's theory (1977) and research findings from Bernal and Froman $(1987,1993)$. The cultural competency and confidence model focuses on provision of a transculturally competent curriculum; evaluation of the effectiveness of certificate courses, campus activities, transcultural resources, and faculty development; and comfort with cultural content. Initially developed for an associate degree nursing program in Staten Island, New York, this model would require further evaluation and psychometric testing with a sample of participants attending a public, minority-serving research-intensive university. This model was expanded to assess cultural self-efficacy of students, faculty, and institutions. Most recently, Jeffreys contributed Chapter 8 of the transcultural nursing and healthcare core curriculum entitled, "Educational Issues for Students, Organizational Staff, Patients, and Communities" (Douglas \& Pacquiao, 2010, pp. 338s-356s).

Cultural-development model (Wells, 2000). Wells developed the culturaldevelopment model (CDM) to assist nurses and enhance the completion of clients' cultural assessments (Wells, 2000). Using Leininger's (1988) guiding principles to support the CDM's conceptual definitions, Wells (2000) stated that, "nurses, other health care professionals, and institutions" have not been successful at incorporating Leininger's principles and this has resulted in the "stagnation of cultural development" (p. 194). Wells also adapted Campinha-Bacote's conceptual definition of open attitudes to diminish stereotypical views. Concepts that compose the CDM are cultural awareness, cultural sensitivity, and cultural competence (Wells, 2000, p. 189). Wells hypothesized 
that increases in cultural awareness, sensitivity, and competency occur on a continuum through affective and cognitive phases. The affective phase includes cultural sensitivity, cultural competence, and cultural proficiency. The cognitive phase includes cultural incompetence, cultural knowledge, and cultural awareness. This model directs healthcare providers to examine their own beliefs for cultural bias or stereotyping that can interfere with culturally congruent care (Wells, 2000).

Matrix for growth through transcultural immersion (Ryan \& Twibell, 2002). The propositions in this model relate to diverse immersions that result in increased growth, high levels of adaptability, and effective communication skills with individuals from different backgrounds. The underlying assumption is that preparation and cultural knowledge are required for adaptation. A high-degree of personal flexibility is said to be associated with effective adaptation. Ryan and Twibell (2002) proposed that increased diversity exposure increases students' and clients' comfort and satisfaction with community health care $(2002$, p. 38). This matrix focuses on providers, patients, and family satisfaction with community healthcare providers and services, and should be incorporated into community-health courses (Ryan \& Twibell, 2002). Focus groups and qualitative inductive data analyses unveiled factors related to cultural competency in community healthcare settings.

\section{Cultural Competence in Healthcare Scale (Caffrey, Neander, Markle, \&}

Stewart, 2005). Caffrey, Neander, Markle, and Stewart (2005) used Wells' (2000) model to define affective and cognitive development of cultural competency, as well as to assist in the creation of the Caffrey Cultural Competence in Healthcare Scale. Wells' (2000) model guided the integration of sexual-orientation content into a nursing curriculum's 
health-assessment course (Eliason \& Raheim, 2000). Wells' (2000) model guided the development of a cultural-learning activities designed to identify cultural issues related to nursing education and research (Leiper, Van Horn, Hu \& Upadhyaya, 2008).

Model of cultural competence (Purnell, 2005). Purnell developed the model of cultural competence in response to the need to teach nursing students how to gather and organize cultural-assessment data (Purnell, 2002; 2005). Purnell's model was drawn as a 12-slice pie. Each slice represented a domain of culture. A circle drawn around the pie includes the person, family, community, and global society. A jagged line drawn along the bottom depicts the nonlinear pattern taken toward cultural competency (Purnell, 2005). This model is best applied to teach undergraduate and graduate nursing students mastery of cultural assessment in any health care setting.

\section{The process of cultural competence in the delivery of healthcare model}

(Campinha-Bacote, 2007). Campinha-Bacote's (2007) worldview and model were influenced by experiences as an ethnically diverse professional mental health nurse. Although these experiences showed some similarity to Leininger's, Campinha-Bacote's (2002) process of cultural competence in the delivery of healthcare model was quite different and much more succinct. The model was initially composed of four constructs: cultural knowledge, cultural sensitivity, cultural awareness, and cultural encounters. The fifth construct of cultural desire was added later, making a total of five interrelated concepts that create cultural competence in healthcare (Campinha-Bacote, 2007). The model was depicted as a volcano with cultural desire spurring the eruption that contained the other four concepts. A recent revision depicts the model constructs with circles drawn in a circle, each one overlapping to demonstrate the philosophical underpinnings of 
cultural competency as an ongoing, dynamic process. Campinha-Bacote's model guided creation of the most popular instrument used in cultural competency research, the Inventory for Assessing the Process of Cultural Competence Among Healthcare Professionals $\left(\operatorname{IAPCC}^{\circledR}\right)(1999 ; 2002)$.

Transcultural-assessment model (Andrews \& Boyle, 2008). Building on the concepts and principles from the works of Leininger and McFarland (2006) and Campinha-Bacote (2002), Andrews and Boyle (2008) developed the transcultural assessment model. This model supports evidenced-based and theoretical assumptions that cultural awareness, knowledge, and sensitivity are required for basic cultural knowledge to evolve; however, it has not guided cultural competency research.

\section{Cultural competency-development model (Papadopoulos, Tilki, \& Ayling,}

2008). To enhance the cultural competency of persons who work with children and adolescents in mental health settings in the United Kingdom, Papadolopous, Tilki, and Ayling (2008) developed the model of cultural-competence development, aimed at delivering team-based, patient-focused, and continuous professional development. The model is viewed as a simple diagram of boxes and arrows to depict the process of continuously evolving constructs of cultural awareness, cultural knowledge, cultural sensitivity, and cultural competency. In this context, cultural awareness connotes selfawareness, identification, and adherence to heritage and ethnocentricity. Cultural knowledge refers to health beliefs, behaviors, stereotyping, and ethnohistory, and knowledge includes understanding of anthropological, sociological, psychological, and biological variations. Cultural sensitivity denotes empathy, interpersonal-communication skills, trust, acceptance, appropriateness and respect; and cultural competency focuses on 
assessment, diagnostic, and clinical skills (Papadopoulos et al., 2008). This model provided conceptual definitions and clinical guidance for the creation of the Children and Adolescent Mental Health Service action tool, designed for culturally competent assessments of children and adolescents in mental health settings. (Papadopoulos et al., 2008).

The health-traditions model (Spector, 2009). Spector (2009) brought a unique view of transcultural nursing with the health traditions model, otherwise known as the heritage model. Symbolized as a staircase, it is assumed that as students ascend the stairs, they also increase in cultural knowledge toward competency. Spector (2009) provided a religious and spiritual perspective of many cultures encountered that merits inclusion in cultural competency education. The dimensions of spirituality, family roles, ceremonies, and objects used in cultural and religious practices are incorporated in this model. Such practices are especially viewed as important during birth, illness, and death. Use of amulets, coining, and other alternative therapies are significant assessment data for healthcare providers to know prior to judgment or decisions. Clients may use combinations of traditional medicine and spiritual healers. Healers may prescribe therapies such as prayers, herbs, or rituals, including the use of fire, strings, or sacrifices to ward off evil or illness-causing spirits. Although this model is used to guide matriculated or continuing education curricula, it has not been applied in nursingeducation research.

Cultural competency model (CCM; Schim \& Doorenbos, 2010). Schim and Doorenbos adapted Leininger's cultural competence and congruent care definitions to develop the cultural competency model (CCM). This model is depicted as a three- 
dimensional figure with each level being comprised of four interlocking puzzle pieces representing concepts (Schim, Doorenbos, Miller, \& Benkert, 2003). The three levels include the provider, the client, and culturally congruent care (Schim et al., 2007). The provider level includes cultural awareness, cultural diversity, cultural sensitivity, and cultural competence (Schim et al., 2007). The client level encompasses individuals, family, and community. The culturally congruent care level requires effective crosscultural interactions and treatment (Schim et al., 2007). The area between the levels represents the environment and other influences on patients' and providers' interactions and health care.

Theoretical assumptions of this model include the affective, cognitive, and behavioral domains of cultural competency (Schim \& Doorenbos, 2010). One assumption requires the desired outcome to be culturally congruent care. It is proposed that cultural competency levels are directly associated with amounts of diverse encounters, exposures, interactions, experiences, and training (Schim et al., 2007). Thus, cultural competency is viewed as a dynamic, ongoing process. The CCM model builds on theoretical definitions and operationalized concepts to develop the associated tool that evaluates cultural competency levels of any healthcare worker level or role (Schim et al., 2003). The unique aspect of this model is its ability to evaluate performed CCBs rather than perceived selfefficacy or intent to perform behaviors.

Summary. Theories and models of cultural competency were developed to organize assessment data, enhance patient data collection, and improve the quality of culturally diverse patient care. There is continuing support for the claim that components of cultural competency include affective, cognitive, and behavioral domains that are 
critical in increasing cultural knowledge, awareness, skills, and competency. Cultural competency models stress the importance of interactions, encounters, experiences, or exposures. Experts asserted that cultural competency levels will be improved with increased exposure to or experience with diversity, and that it is more important to identify similarities among cultures than differences.

\section{Research on Cultural Competency}

Cultural competency research in nursing began over 20 years ago in the United States with the American Nurses Association mandate for incorporation of cultural content in nursing curricula and practice (American Nurses Association, 1986). Leininger's theory guided much of the research on culturally competent curricula (Cortis, 2000; Gebru, Ahsberg, \& Willman, 2007). Research in cultural competency and nursing often referred to Leininger's theory, which remains as a guide for qualitative research (Cortis, 2000; Leininger, 1988; Mixer, 2011). Leininger's theory was infrequently used to create a quantitative tool and none became popular (Baldonado et al., 1998). The theory was specifically developed to guide qualitative ethnographic research, and a major goal is ongoing transcultural education and qualitative research that is documented and shared (Leininger, 1995; Mixer, 2008).

Leonard (2006) used Leininger's sunrise model and qualitative method to guide a three-step analysis of NLN accredited-schools curricula, including evidence of cultural content threaded or stranded throughout. Results showed accredited schools documented cultural content in their curricula, but Leonard (2006) was unable to evaluate or conclude its actual implementation and effectiveness. Ryan et al. (2000) used Leininger's theory for cultural competency definitions in the creation of a quantitative tool for faculty to 
examine whether transcultural nursing content and planned student experiences were in use. Ryan et al. (2000) focused on community-health nursing education, stating that it is the frontline of diverse, vulnerable, and poor patient care. More research with this model could evaluate community-health curricula and increases in novice nurses' knowledge, while stimulating an interest in this setting as a potential practice site.

Although Leininger's theory is mentioned several times to support cultural competency definitions, the model did not produce a quantitative tool that would become popular. Other scholars felt compelled to develop their own models and associated tools (Baldonado et al., 1998; Bernal \& Froman, 1987; A. M. Brennan \& Cotter, 2008; Caffrey et al., 2005; Campinha-Bacote, 1999; Goode et al., 2006; Jeffreys, 2000; Schim et al., 2007). Bandura's (1977) self-efficacy theory guided the development of Bernal and Froman's (1987) quantitative tool, the Cultural Self-Efficacy Scale (CSES).

Bernal and Froman (1987) developed the CSES based on cultural knowledge, cultural patterns, and cultural-skills concepts. They compared the independent variables of educational levels, age, years of experience, with self-efficacy to care for three ethnically diverse groups. The CSES was used frequently in cultural self-efficacy research (Alpers \& Zoucha, 1996; Bernal \& Froman, 1993; Hagman, 2006; Kulwicki \& Boloink, 1996; Smith, 1998; St. Clair \& McKendry, 1999). Generally, studies using the CSES lacked "predictive power" associated with demographic variables (Bernal \& Froman, 1987, p. 202). Nurses and students consistently showed a lack of self-efficacy to care for culturally diverse clients (Bernal \& Froman, 1987, 1993; Kulwicki \& Boloink, 1996). Based on the meta-analysis of Coffman, Shellman, and Bernal (2004) concerning the use of the CSES, recommended that Jeffrey's (2000) cultural competency and 
confidence model and the Transcultural Self-Efficacy Tool be used in future studies of transcultural self-efficacy (p.180). The Transcultural Self-Efficacy Tool demonstrated good psychometric evaluation with associate degree nursing-student populations (Jeffreys, 2000; 2010; Jeffreys \& Dogan, 2012).

Hagman (2006) continued to use the CSES and evaluated the cultural selfefficacy levels of nurses in the southwest. Those RNs worked in hospitals and showed moderate levels of self-efficacy to care for patients from three selected diverse groups (Hagman, 2006, p.107). The study participants who reported they had prior knowledge of Leininger's theory had higher self-efficacy scores (Hagman, 2006). Limitations of the CSES are that only three ethnicities can be compared and participants report self-efficacy or confidence to perform culturally competent care rather than reporting actually performed care.

As the U.S. Census Bureau (2010) continued to report increases in diversity. Licensing and accrediting agencies also reported increases in diversity of RNs and nursing students (Bond, 2004; Buerhaus, 2008; USDHHS, 2010); generational differences and diversity were observed in classrooms and workplaces. Buerhaus (2008) predicted that RNs over 50 years old would be the largest group in the workforce by 2010 . The greatest obstacles for American public health nurses were language, communication, and the effective use of interpreters to collect accurate data and provide patient education (Starr \& Wallace, 2009). The poor communication skills of many foreign-born RNs hindered their care of American English-speaking patients, especially in decision making and discharge teaching (Guttman, 2004). 
Using the CCM and CCA, Schim, Doorenbos, and Borse (2005) conducted a study of the cultural competency of nurses from Canada and the United States (Michigan). Their findings showed that CCBs were significantly associated with cultural competency training $(p=.002)$, higher educational attainment $(p<.001)$, and country $(p=.016)$. Prior cultural competency training and educational level were significantly associated with cultural knowledge, awareness, and sensitivity. There were no significant associations found between cultural competency levels and years of experience, numbers of diversities encountered, self-identified race/ethnicity, discipline, country, or age (Schim et al., 2005). Also, there were no significant differences in cultural competency levels between the Canadian and American nurses (Schim et al., 2005). The CCA reliability indices in this sample had Cronbach's alphas of .89 for the total scale, .76 for the CAS subscale, and .93 for the CCB subscale (Schim et al., 2005).

Studies in other countries shed more light into the phenomena of cultural competency in healthcare delivery. In 1998, Ehrenfeld, Shmueli, and Henig reported that parents' educational level, occupational status, and sociodemographic status were significantly associated with immigrant-nursing students' perceptions of nursing in Israel. Analysis showed that immigrant parents usually held university degrees and native Israeli parents had mostly obtained high school diplomas (Ehrenfeld, et al., 1998). However, the study evaluated only students' communication, language, and academic skills, even though cultural competency was part of the curriculum (Ehrenfeld et al., 1998). In 2008, Graham and Richardson published a study using gaming to increase students' cultural awareness in the U.K. Whereas qualitative findings had not supported increased cultural 
awareness, important facts on how to improve gaming as a teaching strategy were presented.

\section{Cultural Competency Research in Nursing Education}

Institutional mandates for program accreditation and adequate preparation for global nursing and state board examinations have triggered nursing education to incorporate cultural content in curricula and teaching-learning activities. In an earlier study, Yoder (1996) evaluated faculty characteristics that were shown to be influential in students' cultural competency levels. It was noted that cultural knowledge, philosophies, and values developed from previous backgrounds had a long-lasting effect on faculty's beliefs. Grossman et al., (1998) evaluated nursing programs with respect to inclusion of cultural competency in nursing curricula, activities, and institutional environments. The data were collected from deans and directors of nursing programs across the United States. Results showed that accreditation requirements were met in cultural threads throughout the various curricula; however, evidence of measured effectiveness or activities actually taking place was too difficult to detect (Grossman et al., 1998). These findings triggered individual nursing programs to evaluate curricula and associated factors with their students' cultural competency levels.

A number of studies have been conducted to investigate students' cultural competency levels and associated factors. Reeves and Fogg (2006) explored 13 students' cultural backgrounds and nursing-program experiences. The students completed Campinha-Bacote's (1999) 20-item IAPCC ${ }^{\circledR}$. Students who scored in the culturally aware range indicated that life experiences were found to have greatly influenced their perceptions of cultural competency (Reeves \& Fogg, 2006). Foreign-born students 
reported that American classrooms were culturally incompetent and showed a lack of cultural sensitivity, even though cultural competency content was incorporated in the curriculum (Junious, Malecha, Tart, \& Young, 2010).

Riley (2010) used the IAPCC-R ${ }^{\circledR}$ to evaluate the cultural competency levels of RN-BSN students and that of their online faculty. The research findings showed a moderately negative relationship between students' years of experience and cultural skill. Age was found to be a significant factor. The 20-39 year-old group and the 41-50-yearold group significantly differed in cultural skill (Riley, 2010). Riley found no significant association between race/ethnicity and students' cultural competency levels (p. 30). Kardong-Edgren et al. (2010) labeled today's students nontraditional; their rationale was that student demographics have changed in age over the past decades, and students are likely to be employed and have responsibilities that conflict with academics as well as younger classmates' technology skills (U.S. Department of Health and Human Services, 2010). Younger students showed a significant correlation to lower IAPCC- $\mathrm{R}^{\circledR}$ scores in cultural awareness than older students; the rationale was that younger students had fewer life experiences and less motivation for cultural competency (Kardong-Edgren et al., 2010). However, these results were not strongly consistent across the six nursing programs compared (Kardong-Edgren et al., 2010). Older students have one advantage over their younger counterparts, with more life experiences to draw on and apply to concepts learned (Bednarz, Schim, \& Doorenbos, 2010, p. 256). These findings support the inclusion of age in any comparison of cultural competency levels among student groups. 
Of note, the NLN (2008) reported that nearly $45 \%$ of the nation's professional nurses and doctoral students combined were from ethnic minorities (Kaufman, 2010). Additionally, the AACN (2008) reported that students from diverse backgrounds accounted for $26 \%$ of all new BSN students. This increasing diversity of nursing students reflects changes occurring in communities (Kaufman, 2010). "Given current demographic trends it is probably unrealistic to assume health care providers can gain indepth knowledge about the health-affecting beliefs and practices of every ethnic or cultural group they are likely to encounter in practice" (Berlin \& Fowkes, 1983, p. 938). Thus, for the benefit of culturally diverse patients and nurses, it was suggested that an extensive orientation of foreign-born and nurses from diverse backgrounds be conducted by culturally sensitive experts, nurse mentors, and preceptors to ease acculturation challenges in Western American academia and healthcare systems (Guttman, 2004; Zeitlin-Ophir, Melitz, Miller, Podoshin, \& Mesh, 2004).

The literature review showed Campinha-Bacote's (2002) framework and IAPCC ${ }^{\circledR}$ are commonly used as guides for the development of cultural competency research. However, many studies reported that Campinha-Bacote's instrument had not performed as well with students as with licensed practicing healthcare professionals. Of note the 25item IAPCC- $\mathrm{R}^{\circledR}$ calculates mean scores and categorizes levels by score ranges. Cultural incompetence ranges from 25 to 50 points; cultural awareness, 51 to 74 points; cultural competence, 75 to 90; and, cultural proficiency, 91 to 100 points (Brathwaite, 2005). The most successful outcomes with the IAPCC- $\mathrm{R}^{\circledR}$ were found in pretest and posttest research designs that measured significant increases in respondents' scores after various cultural educational interventions (Brathwaite, 2005, 2006; Doutrich \& Storey, 2004; Fahrenwald, 
Boysen, Fischer, \& Maurer, 2001; Hunter, 2008; Hunter \& Krantz, 2010; KardongEdgren, 2007; Kardong-Edgren \& Campinha-Bacote, 2008; Kardong-Edgren et al., 2010; Larson, Ott, \& Miles, 2010; Reneau, 2013; Sargent, Sedlack, \& Martsolf, 2005). Campinha-Bacote's IAPCC- $\mathrm{R}^{\circledR}$ was also tested in a study with baccalaureate faculty across the United States; mean scores were found to fall within the culturally competent range (Kardong-Edgren, 2007; Wilson, Sanner, \& McAllister, 2010). This finding was in contrast to previous reports that nurses and students consistently lacked confidence or self-efficacy (Bernal \& Froman, 1987; 1993; Kulwicki \& Boloink, 1996). This inconsistency is important given that faculty remain an integral aspect in the teaching of cultural competency and the levels that students attain (Reneau, 2013).

The research findings of a study that compared the cultural competency levels of faculty and BSN students who were in the 1st and 4th years of their program merit consideration. The findings highlighted the importance of the need for more structured cultural content in clinical and faculty preparation (Sargent et al., 2005). Sargent et al. (2005) found that 4th-year nursing students had significantly higher perceived cultural competency than 1st-year students (p. 218). Participants' personal experiences with other cultures influenced perceptions and self-efficacy toward performing effective transcultural nursing care (Lim, Downie, \& Nathan, 2004, p. 432). However, Lim et al. (2004) found that 1st- and 4th-year nursing students' perceptions of transcultural selfefficacy was not significantly associated with age, gender, birth country, current employment, or language spoken at home.

Researcher evaluation of specific strategies that attempted to increase cultural knowledge, awareness, behaviors, and competency showed that targeted interventions 
have some effect on awareness or competency levels. Researchers found reading literature and discussions had positive outcomes (Anderson, 2004; Bartol \& Richardson, 1998; Clark, Zuk, \& Baramee, 2000). Students read The Spirit Catches You and You Fall Down (Fadiman, 1997) and showed improvement in their cultural awareness and sensitivity (Anderson, 2004). Campinha-Bacote's model guided the course development and evaluation with a pre- and posttest design and narrative content analysis, and reported that any combination of books produced positive feedback (Anderson, 2004).

Halloran (2009) used reading, written assignments, and class discussions as strategies in a cultural nursing course. Novels were chosen to stimulate students' reflections and increase their self-awareness "knowledge, understanding, and compassion" (Halloran 2009, p. 524). However, the students thought the books encouraged stereotypical thinking, and the faculty found that class management was very difficult because students were at different reading points of reference (Halloran, 2009). Analysis of students' comments showed complaints on the difficult reading level and that assignments took much longer then they had anticipated (Halloran, 2009). Although the book list was phenomenal for enrichment of any culturally focused course, the author did not report quantitative evaluation (Halloran, 2009).

Authors found consistent increased cultural competency levels after students participated in international immersions and local community-health diverse client-care experiences (Amerson, 2010; Caffrey et al., 2005; Heuer, Bengiamin, \& Downey, 2001; Kollar \& Ailinger, 2002; Larson et al., 2010; Lockhart \& Resick, 1997; Reneau, 2013; Riner \& Becklenberg, 2001; St. Clair \& McKendry, 1999; Walsh \& DeJoseph, 2003; Zorn, 1996). St. Clair and McKendry (1999) found that nursing students, regardless of 
their age or experience, showed increased cultural sensitivity and competency after international immersions. Alpers and Zoucha (1996) compared the cultural confidence of senior nursing students, some of whom had received cultural content in a community health course. Results showed the group that received the cultural content reported higher confidence levels and cultural knowledge of diverse lifestyles and employment patterns. Results consistently supported the hypothesis that diverse ethnicity or race is not significantly associated with cultural competency levels but that experiences, exposure, and immersions are directly associated with cultural competency levels. Kollar and Ailinger (2002) asserted that international experiences are an excellent opportunity to increase students' "global perspective and enhance their cultural competency" (p. 28).

Walsh and DeJoseph (2003) explored the experiences of 10 nursing students and two instructors after residing in a remote region of Guatemala for 2 weeks. Students were selected through an application process and preference was given to those fluent in Spanish and good at fundraising. Analysis of student data supported that an increase in students' cultural competency levels was possible with shorter international immersions (Walsh \& DeJoseph, 2003). These findings lend support for earlier findings reported by Kavanagh, Absalom, Beil, and Schliessmann (1999). They observed an increase in students' cultural competency levels after "intercultural immersions." This resulted in their development of a participatory research model, which provides a cross-cultural communication guide to use with clients from diverse background to improve quality and patient outcomes (Kavanagh et al., 1999). Bond and Jones (1994) recommended immersions be more than 2 weeks in length to achieve any lasting learning outcomes. However, researchers found inconsistent evaluation of the long-term impacts on nursing 
students' cultural competency levels after international immersions (Kollar \& Ailinger, 2002; p. 31).

Caffrey et al. (2005) evaluated nursing student groups' perceptions of cultural competency after completing international and local diversity immersions. The study showed that public health or local diverse experiences and international travel groups led to increases in cultural self-efficacy, and greater gains were observed consistently with international groups (Amerson, 2010; Caffrey et al., 2005; Callister \& Cox, 2006; Duffy, Farmer, Ravert, \& Huittinen, 2003). Memmott et al. (2010) described serious coordination challenges for sustainable international immersions. Faculty and administrators need to consider obtaining institutional support and allowing faculty time for tenure activities (Memmott et al., 2010). Use of community health courses and local exposures often remain the most successful and cost-effective choice.

Similarly, Ryan and Twibell (2002) found that local diverse field immersions relate strongly and directly to students' cultural competency outcomes. This outcome resulted in their development of the model for transcultural nursing immersion experience and the Transcultural Nursing Immersion Experience Questionnaire (p. 32). However, both the model and tool have not yet become popular in nursing education and cultural competency research. S. J. Brennan and Schulze (2004) reported the challenges experienced in coordinating community health courses. Analysis of narrative writing showed students made progress in critical-thinking and ethnocentricity (S. J. Brennan \& Schulze, 2004). Readings, discussions, and presentations were useful in preparing students for the practicum community-service component (S. J. Brennan \& Schulze, 
2004). Doutrich and Storey (2004) also found community health courses that used public health nurse mentors for students significantly increased cultural competency levels.

Researchers have used one-day seminars and continuing education to increase cultural competency. Sanner, Baldwin, Cannella, Charles, and Parker (2010) evaluated the effects of a 45-minute Diversity Forum. Faculty and culturally diverse community members interacted with students in small groups. Using a pre- and posttest research design, students completed the Openness to Diversity/Challenge Scale survey (Sanner et al., 2010). This strategy may be effective at increasing openness to diversity (Sanner et al., 2010). Rooda and Gay (1993) evaluated participants' comments after a 1-day staffdevelopment workshop and found an increase in administrators' cultural-sensitivity levels. Supportive comments included that more "time and resources" would be allocated to cultural competency (Rooda \& Gay, 1993, p. 265).

Doorenbos et al. (2010) developed a web-based cultural competency continuingeducation course and evaluated it for effectiveness as a format and for increasing cultural awareness and competency of providers. Evaluation of the web-based platform showed positive outcomes (Doorenbos, et al., 2010). This research was deemed important as it used the three-dimensional CCM to underpin it; trends showed nursing education will continue to increase the use of technology in web-based courses (AACN, 2008; NLN, 2008). Campbell-Heider et al. (2006) evaluated a family-nurse-practitioner curriculum for cultural competency. Surveys and focus-group data showed coursework alone was not adequate; however, immersion and experiential learning methods helped achieve increased cultural competency levels (Campbell-Heider et al., 2006; Hughes \& Hood, 2007). 
Research findings lend consistent support for a variety of methods to be used in classroom and clinical settings to increase affective, cognitive, and behavioral or competent skills. Tuck, Moon, and Allocca (2010) used Campinha-Bacote's model to create a culturally competent advanced-practice-nurse modular curriculum. The curriculum was improved by implementing many strategies such as gaming, case histories, class exercises, group assignments, and active discussions for distance learners. Comparisons of groups using the IAPCC- ${ }^{\mathbb{O}}$ showed a significant increase in the 5 th model construct, cultural desire (Tuck et al., 2010). Benkert, Tanner, Guthrie, Oakley, and Pohl (2005) surveyed four graduate nursing programs to assess differences in students' meaning of cultural competency and to attempt prediction of practice locations (rural, urban, or inner city). Gender was excluded in this study to maintain confidentiality. The predominantly White female sample, aged 31 to 39, reported moderate levels of cultural knowledge and CCB (Benkert et al., 2005). Findings further supported the use of experiential learning to increase cultural competency levels (Benkert et al., 2005).

Kennedy, Fisher, Fontaine, and Martin-Holland (2008) evaluated a university nursing program's diversity content in courses throughout the curriculum. A "mixedmethod, four-step approach" was used to evaluate goals and effectiveness of the curriculum revision (Kennedy et al., 2008). Evaluations and feedback from students and faculty showed that $14 \%$ of the courses had diversity content, the sociocultural courses scored highest in cultural competency, and clinical courses scored lowest (Kennedy et al., 2008, p. 367). The researchers inferred that clinical instructors' lack of comfort with cultural content was the reason for this outcome. Cuellar et al. (2008) revised a university nursing program's curriculum to include cultural threads, strands, and cultural courses, 
and evaluated its effectiveness with The Blueprint for Integration of Cultural Competence in the Curriculum Questionnaire (Cuellar et al., 2008). The data were collected over 2 years from freshman students, graduating seniors, and master's students. The freshmen depicted the lowest exposure levels and insufficient content, but the seniors depicted the highest cultural-knowledge levels (A. M. Brennan \& Cotter, 2008; Tulman \& Watts, 2008). Qualitative feedback showed the faculty had a lack of previous cultural conceptual exposure, were redundant, and used too many didactic methods (A. M. Brennan \& Cotter, 2008). The Cronbach's alpha of the instrument with this sample was 96 (Tulman \& Watts, 2008).

Green, Comer, Elliot, and Neubrander (2011) evaluated undergraduate and graduate nursing students' cultural competency outcomes after an international, servicelearning experience. The researchers used the CCA with multidisciplinary healthcare team members who cared for people in remote areas of Honduras over a 10-day period in a "mobile health unit" (Green et al., 2011, p. 304). Although the sample size was too small for statistical significance, the CCB subscale showed huge gains, indicating positive increases in cultural competency levels from pre- to posttest evaluation (Green et al., 2011). Researchers significantly associated those reporting previous diversity exposure or training with higher cultural competency levels (Schim \& Doorenbos, 2010).

\section{Research on the Cultural competency Model (CCM) and Cultural competency}

\section{Assessment (CCA)}

Guided by the CCM, the CCA was first developed in a four-phase psychometric evaluation (Schim et al., 2003). The last phase was a pilot test with 113 multidisciplinary hospice workers. Factor analysis of the instrument resembled the 25 -item tool used today 
(CCA, Version 2009). Item score range increased from five to seven Likert-type choices. The total CCA scale Cronbach's alpha was .92. Data analysis supported construct validity for the total CCA using criterion related validity with the IAPCC $^{\bigcirc}$ which had Cronbach's alpha of .67 with that group (Schim et al., 2003, p. 36). The CCB subscale Cronbach's alpha was .93 and .75 for the CAS subscale (Schim et al., 2003). The total CCA was moderately correlated, $r=0.66(p=.004)$ with Campinha-Bacote's 20-item IAPCC ${ }^{\odot}$ (Schim et al., 2003). Construct validity was further supported by a two-tailed $t$-test, that demonstrated those with prior diversity training had significantly higher scale scores than those without prior training, $r(90)=2.12(p=.004)$. Bonferroni post hoc analysis revealed those with high school diplomas had significantly lower total CCA scale scores than those with baccalaureate degrees ( $p=.001$; Schim et al., 2003, p. 36). The CCA took less than 15 minutes to complete for the majority of subjects ( $64 \%$ of the total number of participants who provided evaluation-form data; $n=109$; Schim et al., 2003, p. 36). Comments on the degree of difficulty of both scales showed the CCA was easier to read and took less time to complete than the $\operatorname{IAPCC}^{\mathcal{O}}$ (p. 36).

Doorenbos and Schim (2004) conducted a descriptive study with 113 participants who varied in age (25-71 years); educational level (high school through graduate level); and clinical role (volunteers, nurses, and doctors). The findings showed significantly different scores between those who reported diversity training and those who had reported no training $(p=.004)$. Post hoc analysis revealed significantly lower cultural competency scores of those with a high school diploma than those with bachelor or graduate degrees (CCA, CAS, and CCB; Doorenbos \& Schim, 2004, p. 31). Lastly, there were no significant differences in cultural competency based on the variables of race, age, 
years of hospice experience, or number of ethnic groups encountered (Doorenbos \& Schim, 2004, p. 31). This study used the CCA tool with five Likert-type choices. The present version of the CCA has a seven-point range. It is deemed valuable to use and test the CCA across multiethnic, multicultural populations with various educational levels and professional roles.

In 2005, Doorenbos, Schim, Benkert, and Borse completed a psychometric evaluation of the CCA with 51 various hospice workers using a quasiexperimental crossover design. The CCA was administered before and after an intervention. The group conducted a principle-axis factor analysis with test-retest reliability. Pearson's productmoment correlation for the total CCA scale was $r=.85(p=.002)$. The CCB and CAS subscales showed correlations of $r=.87$ and $r=.82$, respectively $(p=.002)$. Findings showed CCA scores were significantly higher among healthcare workers who reported prior diversity training than those that who did not. A subsequent psychometric evaluation of the CCA was completed with a larger sample of healthcare providers $(n=405)$. The Cronbach's alpha of the total CCA with this sample was .89 (Doorenbos et al., 2005). Unpaired, two-tailed $t$-tests showed significantly higher total CCA scores from those who reported prior diversity training, compared to those who had reported none $(p<.001)$. Similarly, results from the quasiexperimental pilot study conducted by Schim, Doorenbos, and Borse (2006b) suggested that short cultural educational interventions may be effective at increasing cultural competency.

Paez, Allen, Carson, and Cooper, (2008) used the CCA to complete a crosssectional study with 23 community-based clinics to evaluate patient-provider communication. Cronbach's alpha with this sample ranged from .50 to .64 (attitude and 
behavioral measures). Simple linear regression showed healthcare workers' cultural competency varied with the amount of diversity at their clinic. Significant associations were found between the clinics' cultural diversity, non-White to White staff ratio, and providers' CCA scores. The clinic's patients and staff diversity were directly associated with higher CCBs. Personal experiences and cultural competency levels were tested in another pilot study, also using the CCA (Starr \& Wallace, 2009). This convenience sample was composed of 31 public health nurses. The 25 -item scale yielded Cronbach's alphas of .90 for the total scale, .67 and .89 for the CAS and CCB subscales, respectively. Descriptive analysis showed the sample was similar in age, but varied in ethnicity and nursing background. One-way ANOVA results showed a significant relationship between participants who had cultural competency training and cultural competency scores (Starr $\&$ Wallace, 2009). Participation in professional seminars was associated with higher CCB scores and overall cultural competency whereas participation in online courses was significantly associated with higher CAS subscale and total CCA scale scores (Starr \& Wallace, 2009, p. 54). Content analysis of three open-ended questions showed past experiences played an important role in perceived cultural competency (Starr \& Wallace, 2009).

Benkert, Templin, Schim, Doorenbos, and Bell (2011) also used the threedimensional CCM and CCA in a cross-sectional descriptive study to evaluate the cultural competency levels of the members of the American Association of Nurse Practitioners. The large sample $(n=474)$ was placed into three minority-nurse groups (Asian American men, African American men and women, and non-Hispanic men). The participants completed five instruments including the CCA. The CAS subscale showed positive 
associations with the CCB subscale using bivariate analysis. Education, nonheterosexual orientation, and diverse life experiences were significantly correlated with higher CAS scores (Benkert et al., 2011). Cronbach's alpha for the total scale with this group was .88, for the CAS subscale $\alpha, .64$, and for the CCB subscale, .92 (Benkert et al., 2011).

Of the models and frameworks reviewed, the three-dimensional CCM was found to be the best to underpin this research. The model was designed for use with healthcare workers with various educational levels, types of backgrounds, and professional or workplace roles. This model is most appropriate for the present study because the projected sample widely differs in educational backgrounds and levels, ethnicity/race, and roles. Models throughout history relied on respondents' self-reported self-efficacy to perform transcultural nursing rather than evaluation of actually performed CCBs (Paez et al., 2008; Starr \& Wallace, 2009), which will be addressed in the present study. As Schim pointed out, there is a need for the CCA too to be tested and evaluated with different cultural groups (S. M. Schim, personal communication, October 25, 2011).

\section{Summary}

Research studies on cultural competency outcomes in nursing education lend support for the assertion that cultural competency is an ongoing process that occurs through phases on a continuum over time. The literature review showed that culturally competent students had higher educational levels and working experience with diverse groups. Researchers asserted that diverse encounters and exposures have direct effects on cultural competency. These diverse experiences must involve interactions of some type, in addition to verbal and nonverbal communication. Studies reviewed demonstrated 
strong support for the three-Dimensional CCM as the underpinning framework for the present study. 


\section{Chapter 3}

Methodology 


\section{CHAPTER 3}

\section{METHODOLOGY}

\section{Introduction}

The theoretical framework that underpins this research is The Three-Dimensional Cultural Competency Model” (Schim \& Doorenbos, 2010). Schim \& Doorenbos (2010) depicted The CCM with three-dimensional jigsaw puzzle pieces on three levels - the provider, client, and the goal of culturally congruent care. The provider level is the focus of this research and contains four components: cultural diversity, cultural awareness or knowledge, cultural sensitivity, and CCBs (Doorenbos \& Schim, 2004). The research questions posed follow:

1. What are the differences in cultural competency levels between undergraduate (RN-BSN Online, Foreign-Educated Physician or FEP BSN, and Traditional BSN) and graduate nursing students (MSN multiple tracks)?

2. To what extent are students' cultural competency levels associated with their program level or academic standing (GPA)?

3. What personal (age, ethnicity/race, gender, language at home) or social factors (previous diversity exposures or previous diversity training) are associated with students' cultural competency levels?

\section{Hypotheses}

1. Higher levels of education are associated with higher cultural competency levels as measured by
a. the CAS
b. the $\mathrm{CCB}$ 
c. the CCA (total scale mean)

d. self-evaluation of cultural competency (rated 1 on the Likert-style scale)

2. Higher program standing (GPA) of students is associated with higher cultural competency levels.

3. Students' cultural competency levels are not associated with the following personal factors:

3.1. age

3.2. gender

3.3. ethnicity/race

3.4. language spoken at home

4. Students' cultural competency levels are associated with the following social factors:

4.1. greater number of diversity encounters over the past 12 months

4.2. previous diversity training

\section{Methods}

Design. This study used a cross-sectional, nonexperimental survey design. An electronic data-collection service called SurveyMonkey was chosen to enhance data collection. The data represented all student groups (program level and specialty track) at one point in time. A 42-item survey captured all data used for analysis.

Sample. This purposive, convenience sample of undergraduate and graduate nursing students was identified as potential participants enrolled at a southeastern, public university during $2013(n=1,139)$. Demographic characteristics were reported as an average age of Traditional BSN (average 29 years-old), RN-BSN average 33 years-old, 
and MSN students' mean age, 35 years old. Seventy to $80 \%$ were expected to be women and $20-30 \%$ were predicted to be men. The ethnicity most often reported was Hispanic, followed by Black, then White/non-Hispanic, with smaller numbers of Asian, Pacific Islander, Native American Indian, and “Other.”

Education characteristics of students included specialty track and level. Undergraduate students were reported to be FEP- BSN ( $n=107)$, RN-BSN Online $(n=256)$, and Traditional BSN $(n=207)$. The total number of MSN students reported was $n=366$ (Fall, 2012). MSN specialty tracks included Adult nurse practitioner (NP; $n=31)$, Child NP $(n=15)$, Family NP $(n=90)$, and Anesthesia Nursing $(n=99)$. MSN students were accepted into the program, taking core courses without a declared specialty track $(n=131)$. Doctoral students $(n=32)$ were excluded from the sample. Power analysis conducted using G-Power for a one-way ANOVA for two groups yielded a medium effect size of 0.25 (partial $\eta^{2}=.06$ ), an alpha of .05 , and power of 0.95 , requiring a sample size of 279 .

\section{Study Site}

The study was conducted on two campuses of a minority-serving public research university located in southeast Miami-Dade County, Florida. These campuses offer undergraduate and graduate nursing programs with major nursing specialties. Graduate students are required to take an advanced culture course (NGR 5131), and undergraduate (BSN) students take required theory and clinical courses that have cultural content threaded throughout the curriculum. Traditional BSN students complete clinical courses with requirements for community-service learning activities and cultural-diversity exposure. 


\section{Instruments}

The Cultural Competency Assessment (CCA) Scale. The quantitative tool used to answer the research questions posed was the CCA scale (Doorenbos et al., 2005). The CCA scale was used in several studies with healthcare providers of varying levels of education and backgrounds. The studies were specifically focused on the evaluation of independent variables and any significant association with groups' cultural competency levels, measured by the CCA scale, the CAS, and the CCB subscales (Doorenbos \& Schim, 2004; Doorenbos et al., 2005; Green et al., 2011, Paez et al., 2008; Schim \& Doorenbos, 2010; Schim et al., 2005; Schim, Doorenbos, \& Borse, 2006a; Starr \& Wallace, 2009).

The 25-item CCA scale was designed to evaluate cultural competency levels measured by the total CCA scale means and subscale means (CAS and CCB). The CAS is composed of 11-items that evaluates cultural awareness, knowledge, and sensitivity in the care of diverse patients. The CCB is a 14-item subscale that evaluates CCBs such as recognizing and removing barriers and seeking cultural information or professional translation services. The CAS and CCB use a seven-point range of Likert-type items. The CAS choices include strongly agree, agree, somewhat agree, neutral, somewhat disagree, disagree, strongly disagree, and no opinion. The CCB subscale uses always, very often, somewhat often, sometimes, few times, never, and not sure. The CAS has some items that are reversed scored whereas the CCB consistently uses always to never with the higher score, 7, as always. Choices of no opinion or not sure were not scored and were treated as missing data. Subscale means were calculated by dividing the sum of the subscale by the number of items answered. The total CCA scale mean was calculated by dividing the sum 
of the two subscales by 2 (Doorenbos \& Schim, 2004). To that end, the higher the CCA, CAS, and CCB means, the greater the cultural competency levels evaluated. Participants completed the four items that evaluate diversity encounters, the 25 -item scale, and finished with demographic items. Diversity encounters and self-identified ethnicity/race allowed for multiple responses. The CCA allows respondents to identify themselves as multiethnic or bilingual with multiple response items.

Demographic survey. Demographic survey choices used drop down menus and check-boxes. Data requested included age, ethnicity/race, gender, and language spoken at home. Ethnicity choices were Hispanic/Latino (including Mexican, Mexican American), Chicano, Puerto Rican, Cuban, other Spanish; White/Caucasian/ European American; Black/African American/Caribbean; American Indian/Alaska Native; Asian (Asian Indian, Chinese, Filipino, Japanese, Korean, Vietnamese, and Other Asian, please specify; Native Hawaiian/Pacific Islander, Arab American/Middle eastern, and, "Other" with a narrative text box limited to 50 characters; see Appendix A).

Educational level in nursing used a drop-down menu with the following choices: Undergraduate BSN or Graduate MSN. Program specialty or track enrolled included the following: Foreign-Physician (FEP) BSN, Traditional BSN, and RN-BSN Online. Graduate level tracks included: FEP BSN/MSN, MSN Adult, Child, and Family Nurse Practitioner, and Anesthesiology Nursing. Track offered "Other, please specify." Program standing was evaluated by a self-reported GPA from a range choices of 2.5-2.9, 3.0-3.4, and, 3.5-4.0. These are unique in grouping variables and rarely included for program standing (GPA) evaluation with cultural competency levels. 
Social factors included the types and the number of times of diverse cultural and special population encounters in different environments or settings over the past 12 months. Previous diversity training was assessed using a drop down menu offering "yes" or "no." After the pilot study, one revision was made with regard to the use of "question logic" after this item. Respondents who chose "no" skipped the two subsequent questions related to previous training. The list of diversity training types included separate college course for credit, content covered in a college course, professional conference or seminar, employer-sponsored program, computer-assisted continuing education, traditional continuing education, and other (specify). Again, the CCA scale allowed for multiple responses to evaluate previous diversity training. The CCA special populations included mentally or emotionally ill, physically challenged/disabled, homeless/housing insecure, substance abusers/alcoholics, sexual orientation, religious/spiritual backgrounds, and other (specify). Number of diversity encounters and training were calculated by a count and a percentage of time/encounters.

Data collection. I received permission to use the CCA scale from its author, Schim (2009, see Appendix B). The program director at the study site and designated staff were contacted to securing consent for their group of students to participate, using electronic mail. After permission was obtained from each program director, the Institutional Review Board (IRB) application was submitted. After IRB approval was secured, e-mails were sent to all undergraduate (BSN) and graduate (MSN) students. The e-mail contained the cover consent letter that explained the research goals and survey process. Two links were provided to potential participants: a link to complete the survey, in which consent would be understood, and a link to "opt-out," to not participate. 
SurveyMonkey and the use of e-mail contact made completion of the surveys private, with no loss of class time or potential coercion of my past or present students. Inclusion of RN-BSN online and MSN core online students was made possible through these datacollection methods.

A unique identifier was created for each e-mail/ISP address and SurveyMonkey identified who completed the survey so they would not receive reminder e-mails. SurveyMonkey provided my account with security and the ability to export data directly into SPSS v.21. Only I possessed the list of e-mail addresses and the unique identifiers assigned; thus all identifying information was kept anonymous and confidential. This information is kept locked and separate from the SPSS dataset.

Psychometric evaluation of the culture-competency assessment. Psychometric testing of the CCA, which was initially a five-point Likert-type tool, was done with a convenience sample $(n=113)$ of multidisciplinary healthcare providers. Choices were always (5) to never (1; Schim et al., 2003). Construct validity was supported by significant correlations $(p<.05)$ found between the CCA and the IAPCC ${ }^{\circledR}$ (Schim et al., 2003). The IAPCC ${ }^{\circledR}$ addressed similar concepts to those of the CCA, such as cultural awareness, cultural knowledge, cultural skill, and cultural encounters. The IAPCC ${ }^{\circledR}$ demonstrated an internal consistency using a Cronbach's alpha of .67 with this sample (Schim et al., 2003). Using factor analysis, the CCA was shown to have an overall internal consistency of Cronbach's alpha of .92 . The CCA subscales performed well with a Cronbach's alpha of .93 for the CCB and an alpha of .75 for the CAS (Schim et al., 2003). Correlation between the IAPCC ${ }^{\circledR}$ and the CCA was moderate $(r=.66$; Schim et al., 2003, p. 36). Contrasted group validity of the CCA with independent variables of 
educational background and previous diversity training were significantly higher among those who reported prior diversity training than those who had no previous training $(r(90)=2.12, p=.004$; Schim et al., 2003). Of respondents, $40 \%$ stated the CCA was easier to comprehend than the IAPCC ${ }^{\circledR}$ and took 15 to 30 minutes to complete (Schim et al., 2003).

Doorenbos et al. (2005) conducted additional psychometric evaluation using tests-retests with a sample of healthcare providers $(n=51)$. The research used a quasiexperimental, crossover design with a control group, with two time points over 4 months. The test-retest method was chosen to examine the effectiveness of a cultural educational intervention. Pearson's product-moment correlation showed the CCA had a significant correlation of $r=.85, p=.002$ (p. 327). The subscales performed equally well with the CCB correlation of $r=.87, p=.002$, and the CAS subscale was $r=.82, p=.002$ (Doorenbos et al., 2005, p. 327).

Continued reliability and construct validity testing was completed with healthcare providers $(n=405)$. The total scale was shown to have a reliability index of Cronbach's alpha of .89 for internal consistency (Doorenbos et al., 2005, p. 328). The reliability for the CCB subscale was a Cronbach's alpha of .91 and was .75 for the CAS subscale, with that sample (Doorenbos et al., 2005, p. 327). Psychometric evaluation of the CCA supported its use for the research questions posed.

Data analysis. SPSS v. 21 was used to conduct statistical tests and analyses. Descriptive statistics and evaluation was conducted by frequency distributions, means, and standard deviations. One-way ANOVAs, Pearson's correlations, and multiple regression models were used to answer the research questions. 
Independent and dependent variables summarized. The independent variables included educational level (BSN or MSN) and several specialty tracks listed above. Program standing referred to self-reported GPAs. Personal or demographic variables included age, gender, language used at home, and self-identified race/ethnicity. Social variables included determination of previous encounters with clients with backgrounds different from the students'. Questions determined the types and number of times (or percentages) of previous diversity encounters and previous diversity training attended over the past 12 months.

The primary dependent variable was cultural competency, evaluated in a few ways. One item used a five-point Likert-like scale as a self-evaluation of overall cultural competency, and used a range from (5) very competent, competent, somewhat competent, somewhat incompetent, and very incompetent (1). Cultural competency was evaluated by use of the total (CCA) scale and (CAS and $\mathrm{CCB}$ ) subscale group means. Evaluation of cultural competency with the CCA demonstrated that higher scale means reflected higher cultural competency levels. Psychometric evaluation demonstrated support for the use of the CCA to evaluate students from various cultural and educational backgrounds.

\section{Hypotheses}

Hypothesis 1. Students enrolled in or reporting higher educational levels will have significantly different or higher cultural competency levels, as measured by the CAS subscale, CCB subscale, and total CCA means. A one-way ANOVA was used to evaluate differences on total CCA, CAS, and CCB subscale means among nursing student groups by level (BSN and MSN) and by specialty tracks (FEP BSN, RN-BSN Online, Traditional BSN, FEP BSN/MSN, MSN ARNP, and MSN Anesthesiology). Post 
hoc tests of significant findings helped to determine exactly which groups were significantly different in scale or subscale means.

Hypothesis 2. Students who report higher program standing (GPAs) are associated with higher cultural competency levels. Pearson's correlations were used to evaluate any significant relationships among student groups by program level, program track, and program standing (GPA) and groups' cultural competency levels (total CCA, CAS, and CCB subscales). One-way ANOVAs were used to determine significant differences among groups' cultural competency levels, program level, and program tracks.

To answer Research Question 3, Hypotheses 3 and 4 were formulated, based on the literature review, to demonstrate the different outcomes expected among personal and social factors and students' cultural competency levels (total CCA, CAS, and CCB subscales).

Hypothesis 3. Students' cultural competency levels are not associated with the following personal factors: age, gender, ethnicity/race, and language used at home. Pearson's correlations were used to determine significant relationships of groups' cultural competency levels, measured by total CCA and CAS and CCB subscales by age, gender, ethnicity/race, and language used at home. One-way ANOVAs were used to determine significant differences among groups' cultural competency (total CCA and CAS and CCB subscales) by gender, ethnicity/race, and language spoken at home groups.

Hypothesis 4. Students' cultural competency levels (total CCA and CAS and CCB subscales) are directly associated with the following social factors: previous diversity encounters and previous diversity training. The statistical tests described above were used to determine any significant relationships among groups' cultural competency 
levels, measured by total CCA and CAS and CCB subscales, previous diversity encounters, and previous diversity training. To further assess significant relationships related to Hypotheses 3 and 4, variables were dummy coded into predictors for use in multiple regression equations. Multiple regressions were used to test for significant amounts of variance explained by the total CCA, and the CAS and CCB subscales.

\section{Handling of Missing Data}

Literature that used the CCA tool did not often publish the handling of missing data. One study only used completed CCA surveys, but did not define the percentage of items answered, to define "complete" (Green et al., 2011). Schim et al. (2005) wrote that surveys should be disqualified if $10 \%$ or more of the information requested was missing (p. 359). Experts have stated that 5 to $10 \%$ of the data missing is the maximum accepted and adopted as the general rule for research (Cohen, Cohen, West, \& Aiken, 2003; Munro, 2005). Pairwise deletion of cases was used and changes in the sample sizes are explained in the results chapter.

\section{Human Subjects and Inclusion Criteria}

Inclusion criteria were enrolled BSN or MSN students at the program site and 18 years of age or older, defined by the NIH as an adult and stipulated in the IRB application for this research. Data were obtained from adult participants only. Students' ability to comprehend the English language was assumed. English is the language used to communicate, read, and learn nursing at this institution. Students' e-mail addresses would be necessary to collect data from participants only once to prevent repeated measures effects. Students might choose not to participate without coercion or penalty and might stop participating at any time. There was no material benefit or compensation for 
participants. Informed consent was assumed by completion of the survey because the cover consent letter explained the study, goals, and informed-consent procedure. No retention strategies were needed because the 30 minutes to complete the survey was the total time involved. The only potential risk for participants was a breach of their survey information or personal information, that is, their student e-mail address.

\section{Data Security}

Anonymity was protected through electronic passcodes and hard copies in locked cabinets. The Gold Level of SurveyMonkey offers higher data security and customer assistance. Data were password protected for computer access. I kept the laptop and any other external memory devices locked in my home office. 


\section{Chapter 4}

\section{Results}




\section{CHAPTER 4}

\section{RESULTS}

The results chapter begins with a section describing the initial screening of data and description of the sample characteristics. The next portion reports the results of the statistical analyses, organized by research questions and hypotheses. The last section summarizes the findings of the study.

\section{Data Screening and Sample Size}

The original data set identified was composed of 1,139 undergraduate and graduate nursing students enrolled at a public, minority-serving university in southeast Florida. A random sample $(n=25)$ was used to pilot the electronic version of the CCA tool. Feedback from the pilot study initiated one change on the electronic CCA tool $\left(\right.$ SurveyMonkey $\left.{ }^{\mathrm{TM}}\right)$. The function was called "question logic" and allowed students who responded "no" to attending previous diversity training, to skip the following two questions that requested information about diversity training attended.

The number of surveys e-mailed to students was 1,114. Reminder e-mails were sent to students every 2 weeks over a total of 4 months (January to April). Two e-mails were returned with invalid addresses, and 22 students chose not to participate. The number of surveys returned was 156 . Participants were deleted if $19 \%$ or more of the survey was incomplete $(n=6)$, or the students' answers were identical across the entire CCA survey $(n=1)$, and if responses were unrealistic or comical $(n=1)$. Deletions were made for missing responses required for grouping variables such as ethnicity/race, program level, program track, and GPAs $(n=4)$. The final sample size was 144 for the 
CAS; however, the sample for the total CCA scale and the CCB subscale decreased to 140 because four students stopped responding at the end of the CAS subscale.

Power analysis for one-way ANOVAs with a medium effect size of $f=0.25$, $\alpha=.05$ between two groups (BSNs and MSNs) with a sample size of $n=144$, resulted in $85 \%$ power using G-Power3. Power analysis for multiple linear regression with an effect size $\left(R^{2}=0.5\right), \alpha=.05,14$ predictors, and a sample size of $n=144$, resulted in $84 \%$ power using G-Power3. To that end, post hoc power analysis found an adequate sample size to support the statistical tests planned: one-way ANOVAs, Pearson's correlations, and multiple linear regressions.

\section{Independent variables.}

Age and gender. This sample was composed of undergraduate and graduate nursing students who had an average age of 32.6 years of age $(S D=9.7)$ and ranged from 20 to 62 years of age. Women $(n=119)$ composed $83 \%$ of the sample, whereas male nursing students accounted for $17 \%$ of this sample $(n=25)$ (see Table 1$)$.

Ethnicity/race. This sample was predominantly Hispanic/Latino (60.4\%; see Table 1). In this survey Hispanic/Latino included Mexican, Mexican American, Chicano, Cuban, Puerto Rican, and other Hispanic/Latino ethnicities. 
Table 1

Gender and Ethnicity/Race of Nursing Students $(n=144)$

\begin{tabular}{|c|c|c|c|}
\hline \multicolumn{2}{|r|}{ Characteristic } & \multirow{2}{*}{$\begin{array}{c}\text { Frequency } \\
119\end{array}$} & \multirow{2}{*}{$\frac{\text { Percentage }(\%)}{83.0}$} \\
\hline Gender & Female & & \\
\hline & Male & 25 & 17.0 \\
\hline \multirow[t]{7}{*}{ Ethnicity/race } & Hispanic/Latino, Mexican, Chicano & 87 & 60.4 \\
\hline & White/Caucasian/European & 45 & 31.3 \\
\hline & African American/Black ${ }^{\mathrm{b}}$ & 24 & 16.7 \\
\hline & $\operatorname{Asian}^{\mathrm{c}}$ & 7 & 4.9 \\
\hline & Native Hawaiian/Pacific Islander & 2 & 1.4 \\
\hline & American Indian/Alaska Native & 1 & 0.7 \\
\hline & Others $^{\mathrm{d}}$ & 5 & 3.5 \\
\hline
\end{tabular}

Note. ${ }^{a}$ Hispanic/Latino included Mexican, Chicano, Cuban, Puerto Rican, and other Latin heritages;

${ }^{\mathrm{b}}$ African American/Black included Caribbean, Haitian, and Jamaican; ${ }^{\mathrm{C}}$ Asian included Asian Indian, Chinese, Filipino, Japanese, Korean, and Vietnamese; ${ }^{\mathrm{d}}$ Other included American Caribbean (2), Cuban American, Eastern Indian and White; Total ethnicity/race is greater than $n=144$ due to multiple responses.

Many students identified with both Hispanic/Latino and White/Caucasian/ European heritages. Therefore, a new ethnic group was created named Hispanic/Latino/White/Caucasian/European $(n=15)$. This reduced the number of Hispanic/Latino students to 72 , or $50 \%$ of this sample (see Table 2). Another new ethnic group was created named Multiethnic $(10 \%, n=5)$, which represented students who identified with two or more ethnicities but were not Hispanic/Latino/White/Caucasian/ European. The majority of the sample chose one ethnicity/race $(n=119), 16.0 \%(n=23)$ chose two, and two respondents chose three (see Table 2). 
Table 2

Multiethnic Characteristics of Nursing Students $(n=144)$

\begin{tabular}{lcc}
\hline \multicolumn{1}{c}{ Ethnicity } & Frequency & Percentage (\%) \\
\hline Hispanic/Latino $^{\mathrm{a}}$ & 72 & 50.0 \\
White/Caucasian $^{\mathrm{b}}$ & 26 & 18.1 \\
African American/Black $^{\mathrm{c}}$ & 22 & 15.3 \\
Hispanic/White/Caucasian/European & 15 & 10.4 \\
Multiethnic $^{\mathrm{d}}$ & 5 & 3.5 \\
Asian $^{\mathrm{e}}$ & 4 & 2.8 \\
\hline
\end{tabular}

Note. ${ }^{a}$ Hispanic/Latino includes Mexican, Mexican American, Chicano, Cuban, Puerto Rican, and other Hispanic/Latino heritage; ${ }^{b}$ White/Caucasian is the same as White/Caucasian/European; ${ }^{\mathrm{c}} \mathrm{African}$ American/Black includes Caribbean, Haitian, Jamaican, and others; ${ }^{\mathrm{d}}$ Groups described in the text above; ${ }^{\mathrm{e}}$ Asian includes Asian Indian, Chinese, Filipino, Japanese, Korean, and Vietnamese.

Language spoken at home. Students spoke primarily English $(n=82)$ or Spanish $(n=54)$, and few chose other languages $(n=8)$. If the student chose two languages, their first menu response was chosen as the primary language and the student was grouped accordingly (see Table 3).

Table 3

Languages Spoken at Home of Nursing Students $(n=144)$.

\begin{tabular}{|c|c|c|}
\hline Language & Frequency & Percentage $(\%)$ \\
\hline English & 82 & 56.9 \\
\hline Spanish & 54 & 37.5 \\
\hline Other $^{\mathrm{a}}$ & 8 & 5.6 \\
\hline
\end{tabular}

\section{Degree level attained, educational level enrolled, program track and standing.}

Educational characteristics were assessed in several ways that included highest degree achieved, enrollment in undergraduate or graduate program levels, program track enrolled, and program standing by self-reported GPA groups (see Table 4). The largest 
group (39.6\%) consisted of those who had attained bachelor degrees in nursing (BSN;

$n=57)$.

Table 4

Highest Degree Attained of Nursing Students $(n=144)$

\begin{tabular}{lcc}
\hline \multicolumn{1}{c}{ Degree/diploma } & Frequency & Percentage (\%) \\
\hline High school diploma & 29 & 20.0 \\
Diploma, LPN/LVN & 1 & 0.7 \\
Associate's degree in nursing & 20 & 13.9 \\
Associate's degree outside nursing & 6 & 4.2 \\
Bachelor's degree in nursing & 57 & 39.6 \\
Bachelor's degree outside nursing & 7 & 4.9 \\
Master's degrees & 10 & 7.0 \\
Doctoral degree (specified) & 14 & 9.7 \\
\hline
\end{tabular}

Note. $\mathrm{LPN}=$ licensed practical nurse; $\mathrm{LVN}=$ licensed vocational nurse.

Students' program level characteristics showed 59\% $(n=85)$ of students were enrolled in undergraduate programs and $41 \%(n=59)$ were enrolled at the graduate level (see Table 5). Undergraduate groups included associate's degree, nursing diploma (LPN/LVN), traditional BSN, and RN-BSN Online student groups. The RN-BSN Online program track students must have had RN licenses for enrollment and courses were entirely Online. Traditional BSN students usually do not have an RN license, have clinical courses with patient encounters, and most courses are offered in traditional/live formats. The FEP BSN students and the FEP Accelerated BSN/MSN students were small samples separately. These two groups have similar curricula, educational backgrounds (medical degrees from outside the United States) and were therefore combined into one group, the FEP BSN/MSN students ( $n=14$; see Table 5). 
Table 5

Educational Level, Program track, and Program Standing (GPA) of Nursing Students $(n=144)$

\begin{tabular}{llcc}
\hline \multicolumn{2}{c}{ Educational characteristics } & Frequency & Percentage \\
\hline Program level enrolled & Undergraduate & 85 & 59.0 \\
& Graduate & 59 & 41.0 \\
Program track enrolled & Traditional BSN & 49 & 34.0 \\
& RN-BSN online & 25 & 17.4 \\
& FEP BSN BSN/MSN & 14 & 9.7 \\
& MSN ARNP & 47 & 32.6 \\
& MSN anesthesiology & 9 & 6.3 \\
Program standing (GPAs) & Lower GPA (2.5-3.4) & 26 & 18.1 \\
& Higher GPA (3.5-4.0) & 118 & 81.9 \\
\hline
\end{tabular}

Note. ${ }^{\text {a }}$ Foreign-educated physician/Bachelor of Science in Nursing/Master of Science in Nursing combined Foreign-educated physician/Bachelor of Science in Nursing and Bachelor of Science in Nursing/Master of Science in Nursing levels; ${ }^{\mathrm{b}}$ Master of Science in Nursing/Advanced Registered Nurse Practitioner combined: Adult $(n=16)$, Child $(n=4)$, and Family $(n=27){ }^{\mathrm{c}}$ Grade-point averages of 2.5-2.9 were combined with Grade-point averages of 3.0-3.4. No students in this sample chose Grade-point averages between 2.0-2.4 (the lowest allowed in the nursing program.

Academic or program standing was evaluated by self-reported GPAs. No students chose GPAs that ranged from 2.0 to 2.4 , although that was a choice on the survey. Only three students chose GPAs of 2.5 to 2.9 , so I decided to combine them with the 3.0 to 3.4 student group (see Table 5).

Previous diversity encounters. I used previous diversity encounters to evaluate students' exposure to culturally diverse and special populations of clients in their healthcare or workplace environments over the past 12 months. Previous diversity encounters were assessed with four questions and all were multiple responses.

Question 1 asked students to select all that applied from the list of ethnicities/races they had encountered including Hispanic/Latino (Chicano, Cuban, 
Mexican/Mexican American, Puerto Rican and Others of Hispanic/Latino heritage);

White/Caucasian/European; African American/Black, Caribbean, Haitian, and Jamaican;

Asian (Asian Indian, Chinese, Filipino, Japanese, Korean, and Vietnamese). All students

$(100 \%)$ responded they had encountered Hispanics/Latinos in their environments; $94 \%$

had encountered Whites/Caucasians, and 94\%, African Americans/Blacks (see Table 6).

Table 6

Diverse Ethnicities/Races Encountered in Nursing Students' Environments with Mean

Percentages Seen $(n=144)$

\begin{tabular}{lcrrrrc}
\hline \multicolumn{1}{c}{ Ethnic/racial groups } & Frequency & $\%$ & $M$ & $S D$ & Minimum & Maximum \\
\hline Hispanic/Latino (others) $^{\mathrm{a}}$ & 144 & 100.0 & 54.0 & 22.9 & 6.0 & 100.0 \\
White/Caucasian & 136 & 94.0 & 18.4 & 13.7 & 0.0 & 65.0 \\
African America/Black (others) $^{\mathrm{b}}$ & 136 & 94.0 & 20.0 & 15.5 & 0.0 & 80.0 \\
Asian $^{\mathrm{c}}$ & 92 & 63.8 & 4.1 & 5.2 & 0.0 & 40.0 \\
Arab/Middle Eastern $_{\text {American Indian/Alaskan }}$ & 62 & 43.0 & 1.5 & 2.4 & 0.0 & 14.3 \\
Native & 28 & 19.4 & 0.8 & 3.0 & 0.0 & 30.0 \\
Hawaiian/Pacific Islander & 22 & 15.3 & 0.4 & 1.3 & 0.0 & 10.0 \\
Other $^{\mathrm{d}}$ & 13 & 9.0 & 0.5 & 2.2 & 0.0 & 20.0 \\
\hline
\end{tabular}

Note. Percentages are greater than $100 \%$ due to multiple response answers; ${ }^{\mathrm{a} H i s p a n i c / L a t i n o ~ i n c l u d e d ~}$ Mexican, Chicano, Cuban, Puerto Rican, and other Latin heritages; ${ }^{\mathrm{b}}$ African American/Black included Caribbean, Haitian, and Jamaican; ${ }^{\mathrm{C}}$ Asian included Asian Indian, Chinese, Filipino, Japanese, Korean, and Vietnamese; ${ }^{\mathrm{d}}$ Other included American Caribbean (2), Cuban American, Eastern Indian and White.

The second survey question instructed students to fill in boxes with the percentages of ethnic/racial clients encountered in their environments. This question requested that the student fill in responses that summed to $100 \%$. Some students' sums were less than or greater than $100 \%$ but were standardized to $100 \%$ for analysis. The mean percentage of student encounters with those of Hispanic/Latino ethnicity in their environments was $54 \%$. The mean percentage of clients encountered who were 
White/Caucasian was $18.4 \%$, and for African American/Black, the mean percentage was $20 \%$ (see Table 6). A new variable, number of ethnic/racial groups encountered, was calculated and used in correlation and multiple linear regression analyses. The mean number of types of ethnic/racial groups encountered, of a maximum of eight types offered, was $3.67(S D=1.56$; see Table 7).

Table 7

Number of Ethnic/Racial Groups Encountered in Nursing Students' Environments $(n=144)$

\begin{tabular}{ccc}
\hline Number of ethnic/racial groups & Frequency & Percentage (\%) \\
\hline 1 & 19 & 13.2 \\
2 & 9 & 6.3 \\
3 & 38 & 26.4 \\
4 & 32 & 22.2 \\
5 & 34 & 23.6 \\
6 & 6 & 4.2 \\
7 & 5 & 3.5 \\
8 & 1 & 0.7 \\
\hline
\end{tabular}

Note. Number of ethnic groups encountered with a maximum of $8 . M=3.67, S D=1.56$.

Question 3 inquired about previous diverse encounters with special-population groups in their environment over the past 12 months (see Table 8). The special populations included mentally or emotionally ill, physically challenged/disabled, homeless/housing insecure, substance abuse/alcoholic, sexual orientation (gay, lesbian, bisexual, and transgendered), different religious/spiritual backgrounds, none of the above, and other (specify). The largest special-population groups encountered were clients with different religious/spiritual backgrounds (79.2\%), followed by client encounters with those who were physically challenged/disabled (72.9\%). In addition, a new variable, the 
number of special populations encountered, was calculated and used in correlation and multiple linear regression analyses. The mean number of all seven types of special populations encountered in students' environments was $3.83(S D=1.83$; see Table 9).

Table 8

Diverse Special Populations Encountered in Nursing Students' Environments ( $n=144)$

\begin{tabular}{lcc}
\hline \multicolumn{1}{c}{ Special populations encountered } & Frequency & Percentage (\%) \\
\hline Different religious/spiritual backgrounds & 114 & 79.2 \\
Physically challenged/disabled & 105 & 72.9 \\
Mentally or emotionally ill & 99 & 68.8 \\
Substance abusers/alcoholics & 84 & 58.3 \\
Gay, lesbian, bisexual, and transgendered & 78 & 54.2 \\
Homeless/housing insecure & 68 & 47.2 \\
None of the above & 10 & 6.9 \\
Other & 3 & 2.1 \\
\hline
\end{tabular}

Note. Percentages are greater than $100 \%$ due to multiple response answers.

Table 9

Number of Special Populations Encountered in Nursing Students' Environments

(Maximum of $7 ; n=144$ )

\begin{tabular}{ccc}
\hline Number of special populations & Frequency & Percentage (\%) \\
\hline 0 & 7 & 4.9 \\
1 & 10 & 6.9 \\
2 & 22 & 15.3 \\
3 & 21 & 14.6 \\
4 & 24 & 16.7 \\
5 & 22 & 15.3 \\
6 & 38 & 36.4 \\
\hline
\end{tabular}

Question 4 assessed previous special population encounters by the percentage of times the student encountered each group in their environment over the past 12 months. 
Although the instructions noted that a sum of $100 \%$ was not required, some students' sums were at $100 \%$, and some were over $100 \%$, which made many responses to Question 4 unusable. This question was not used.

Previous diversity training. Previous diversity training was evaluated in several ways. Students first responded to a dichotomous question of whether they had ever participated in diversity training ("yes" or "no"). Of this student sample, $40 \%(n=52)$ responded "yes," that they had attended previous diversity training (see Table 10). Students who responded "no" skipped the next two questions, which described previous diversity training attended, but were entered as zeros for inclusion in the correlation and multiple linear regression analyses.

Table 10

Previous Diversity Training and Types of Diversity Training Nursing Students Attended $(n=144)$

\begin{tabular}{llcc}
\hline \multicolumn{2}{c}{ Diversity training } & Frequency & Percentage (\%) \\
\hline Previous diversity training & Yes & 92 & 63.9 \\
& No & 52 & 36.1 \\
Diversity training types & Content in a college course & 58 & 40.3 \\
& Employer-sponsored program & 46 & 31.9 \\
& Professional conference/seminar & 28 & 19.4 \\
& Continuing education & 24 & 16.7 \\
& Separate college course for & 22 & 15.3 \\
& credit & & 11.8 \\
& Online (computer-assisted) & 17 & 3.5 \\
& education & & \\
& Other (workshops, etc.) & 5 & \\
\hline
\end{tabular}

Students who responded "yes" were asked to choose which of the types of diversity training listed they had ever attended (see Table 10). Types of diversity training 
included a separate college course for credit, content covered in a college course, a professional conference or seminar, an employer-sponsored program, online (computerassisted) education, continuing education, and "other, please specify." The two most attended types of diversity training attended were content in a college course (40.3\%) and employer-sponsored program (31.9\%). I calculated the number of types of diversity training (of a maximum of 7; see Table 11). The mean number of types of diverse training attended was $M=1.39(S D=1.43)$.

Then students who responded "yes" were asked to choose how many times they had attended each type of diversity training. The mean number of times students had attended all types of diversity training was $M=4.47(S D=7.64)$ and ranged from 0 to 60 .

\section{Table 11}

Number of Types of Previous Diversity Training Attended by Nursing Students ( $n=144)$

\begin{tabular}{ccc}
\hline Diversity training types & Frequency & Percentage (\%) \\
\hline 0 & 52 & 36.1 \\
1 & 33 & 22.9 \\
2 & 27 & 18.8 \\
3 & 22 & 15.3 \\
4 & 6 & 4.2 \\
5 & 2 & 1.4 \\
6 & 1 & 0.7 \\
7 & 1 & 0.7 \\
\hline
\end{tabular}

Note. Maximum of 7 types, $M=1.39, S D=1.43$.

Dependent variables. Total CCA, CAS, CCB subscales. The first 11 items of the CCA scale were scored from strongly agree (7) to strongly disagree (1) and was the CAS subscale (see Table 12), a measure of students' perceived CAS scores. Items with which students most agreed included believing that everyone should be treated with respect 
$(M=6.9)$, and perceiving that knowledge about clients' different cultures had helped them direct their work $(M=6.6)$. This student sample scored lowest on items related to stereotypical thinking, such as race being the most important factor in the determination of a person's culture $(M=4.6)$, and that people with common cultural backgrounds would think and act alike $(M=4.3)$. The two lowest means were reversed scored items on the CAS subscale.

Table 12

Cultural Awareness and Sensitivity Subscale Characteristics of Nursing Students $(n=$ 144)

\begin{tabular}{|c|c|c|c|c|}
\hline Scale items & $M$ & $S D$ & Minimum & Maximum \\
\hline Cultural Awareness and Sensitivity subscale & 6.1 & 0.5 & 4.8 & 7.0 \\
\hline Believes that everyone should be treated with respect & 6.9 & 0.5 & 2.0 & 7.0 \\
\hline Knowing about different cultures helps me direct my work & 6.6 & 0.6 & 4.0 & 7.0 \\
\hline $\begin{array}{l}\text { Understand people from different cultures define concept of } \\
\text { "health care" in different ways }\end{array}$ & 6.5 & 0.6 & 4.0 & 7.0 \\
\hline $\begin{array}{l}\text { Aspects of cultural diversity need to be assessed for each } \\
\text { individual, group, and organization }\end{array}$ & 6.4 & 0.7 & 4.0 & 7.0 \\
\hline $\begin{array}{l}\text { If I know about person's culture, I don't need to assess } \\
\text { his/her personal preferences for health services (rev) }\end{array}$ & 6.4 & 0.8 & 1.0 & 7.0 \\
\hline $\begin{array}{l}\text { Spirituality and religious beliefs are important aspects of } \\
\text { many cultural groups }\end{array}$ & 6.3 & 0.9 & 1.0 & 7.0 \\
\hline Many aspects of culture influence health and healthcare & 6.3 & 0.8 & 2.0 & 7.0 \\
\hline $\begin{array}{l}\text { Individual people may identify with more than one cultural } \\
\text { group }\end{array}$ & 6.2 & 0.8 & 2.0 & 7.0 \\
\hline $\begin{array}{l}\text { Language barriers are the only difficulties for recent } \\
\text { immigrants to the United States (rev) }\end{array}$ & 6.1 & 1.1 & 1.0 & 7.0 \\
\hline $\begin{array}{l}\text { Race is most important factor in determining a person's } \\
\text { culture (rev) }\end{array}$ & 4.6 & 1.7 & 1.0 & 7.0 \\
\hline $\begin{array}{l}\text { People with a common cultural background think and act } \\
\text { alike (rev) }\end{array}$ & 4.3 & 1.6 & 1.0 & 7.0 \\
\hline
\end{tabular}

Note. 7 strongly agree, 2 agree, 3 somewhat agree, 4 neutral, 5 somewhat disagree, 6 disagree, 1 strongly disagrees; rev $=$ reverse scored. 
The next 14 items of the CCA scale were scored from always (7) to never (1) and is the CCB subscale. The CCB subscale items focus on actions and behaviors performed that demonstrate culturally competent care of clients with different backgrounds and preferences (see Table 13). The items performed most often were "I find ways to adapt my services to individual and group cultural preferences" $(M=5.9)$, and "I welcome feedback from clients about how I relate to people from different cultures" $(M=5.9)$. The items performed least often were "I have resource books and other materials available to help me learn about people from different cultures" $(M=4.4)$, and "I use variety of sources to learn about cultural heritage of others" $(M=4.6)$. The CCB subscale does not have any items that are reverse scored.

The total CCA scale is the mean of the CAS and the CCB subscale scores. For this sample, the total CCA scale mean was $5.7(S D=0.7)$ and ranged from 3.54 to 6.86 .

Total CCA, CAS, and CCB subscale reliabilities. Reliabilities by Cronbach's alpha were excellent for the total CCA scale and the CCB subscale (see Table 14). Cronbach's alpha for the CAS subscale was lower but consistent with literature and supported the difficulty in the evaluation of the affective domain of cultural competency. Analysis showed no increase in alphas would occur with the removal of any of the items on the scales (see Table 14.). 
Table 13

Cultural Competent Behaviors Subscale Characteristics of Nursing Students $(n=140)$

\begin{tabular}{|c|c|c|c|c|}
\hline Scale items & $M$ & $S D$ & Minimum & Maximum \\
\hline CCB subscale & 5.2 & 1.1 & 1.92 & 7.0 \\
\hline $\begin{array}{l}\text { I find ways to adapt my services to individual and group } \\
\text { cultural preferences }\end{array}$ & 5.9 & 1.2 & 2.0 & 7.0 \\
\hline $\begin{array}{l}\text { I welcome feedback from clients about how I relate to people } \\
\text { from different cultures }\end{array}$ & 5.9 & 1.6 & 1.0 & 7.0 \\
\hline I avoid generalizations to stereotype groups of people & 5.8 & 1.4 & 1.0 & 7.0 \\
\hline $\begin{array}{l}\text { I remove obstacles for people of different cultures when } \\
\text { people identify barriers to me }\end{array}$ & 5.5 & 1.5 & 1.0 & 7.0 \\
\hline $\begin{array}{l}\text { I recognize potential barriers to service that might be } \\
\text { encountered by different people }\end{array}$ & 5.5 & 1.3 & 2.0 & 7.0 \\
\hline $\begin{array}{l}\text { I remove obstacles for people of different cultures when I } \\
\text { identify barriers to services }\end{array}$ & 5.3 & 1.5 & 1.0 & 7.0 \\
\hline $\begin{array}{l}\text { I include cultural assessment when I do individual or } \\
\text { organizational evaluations }\end{array}$ & 5.3 & 1.6 & 1.0 & 7.0 \\
\hline I document cultural assessments if I provide direct services & 5.2 & 1.9 & 1.0 & 7.0 \\
\hline $\begin{array}{l}\text { I document adaptations I make with clients if I provide } \\
\text { services }\end{array}$ & 5.1 & 1.8 & 1.0 & 7.0 \\
\hline I ask people to tell me expectations for health services & 5.0 & 1.8 & 1.0 & 7.0 \\
\hline $\begin{array}{l}\text { I seek information on cultural needs when I identify new } \\
\text { people }\end{array}$ & 4.9 & 1.7 & 1.0 & 7.0 \\
\hline $\begin{array}{l}\text { I ask people to tell me their own explanations of } \\
\text { health/illness }\end{array}$ & 4.8 & 1.8 & 1.0 & 7.0 \\
\hline $\begin{array}{l}\text { I use variety of sources to learn about cultural heritage of } \\
\text { others }\end{array}$ & 4.6 & 1.8 & 1.0 & 7.0 \\
\hline $\begin{array}{l}\text { I have resource books and other materials available to help } \\
\text { me learn about people from different cultures }\end{array}$ & 4.4 & 2.0 & 1.0 & 7.0 \\
\hline
\end{tabular}

\section{Table 14}

Total Cultural Competency Assessment, Cultural Awareness and Sensitivity, and Cultural

\section{Competent Behaviors Reliabilities}

\begin{tabular}{lcccccc}
\hline \multicolumn{1}{c}{ Total and subscales } & $n$ & $\alpha$ & $M$ & $S D$ & Minimum & Maximum \\
\hline Total CCA (25 items) & 140 & .90 & 5.65 & 0.69 & 3.54 & 6.86 \\
CAS (11 items) & 144 & .64 & 6.07 & 0.47 & 4.82 & 7.00 \\
CCB (14 items) & 140 & .92 & 5.23 & 1.12 & 1.92 & 7.00 \\
\hline
\end{tabular}

Note. $\mathrm{CCA}=$ Cultural Competency Assessment; CAS = Cultural Awareness and Sensitivity; CCB = Culturally Competent Behaviors; subscale sample sizes varied from $n=140$ to $n=144$. 
In addition, perceived cultural competency was measured by one five-point Likert scale item. This one item ranged from (5) very competent to (1) very incompetent. Nearly $50 \%$ of students evaluated themselves as very competent (see Table 15). For analysis, "very competent" was compared to all other levels.

Table 15

Self-Evaluated Cultural Competency Levels of Nursing Students $(n=144)$

\begin{tabular}{lcc}
\hline Self-evaluated cultural competency & Frequency & Percentage (\%) \\
\hline Very competent & 71 & 49.3 \\
Somewhat competent & 64 & 44.4 \\
Neither competent nor incompetent & 9 & 6.3 \\
Somewhat incompetent & 0 & 0.0 \\
Very incompetent & 0 & 0.0 \\
\hline
\end{tabular}

Note. $M=4.43 ; S D=0.61$.

\section{Hypotheses and Findings}

\section{Hypothesis 1: Levels of nursing education and cultural competency. This}

hypothesis stated that students enrolled in higher levels of nursing education (MSN) would have significantly higher cultural competency levels (measured by the total CCA and CAS and CCB subscale scores) than undergraduate-level (BSN) students. In addition, it was hypothesized that higher levels of nursing education students (MSN) would evaluate themselves significantly higher in cultural competency than lower educational level students (BSN).

One-way ANOVAs were used to assess students' cultural competency levels (as measured by the total CCA scale, and the CAS and CCB subscales) for differences between BSN and MSN student groups and among groups by program tracks. Program track groups included FEP BSN/MSN, RN-BSN Online, Traditional BSN, MSN ARNPs, 
and MSN Anesthesiology. A significant difference in CAS, measured by the CAS subscale, was found between BSN and MSN student groups (see Table 16). Graduate level (MSN) nursing students' CAS subscale mean $(M=6.18)$ was significantly higher than undergraduate $(\mathrm{BSN})$ students' mean $(M=5.99), p=.016$. However, $\mathrm{BSN}(M=$ $5.59,5.19)$ and MSN $(M=5.73,5.29)$ student groups did not significantly differ on the total CCA scale or CCB subscale scores, respectively $(p>.05)$.

Table 16

Means of Total Cultural Competency Assessment Scale, Cultural Awareness and Sensitivity, and Cultural Competent Behaviors Subscales by Program level

\begin{tabular}{|c|c|c|c|c|c|c|c|c|c|}
\hline \multirow[t]{2}{*}{ Variable } & \multicolumn{3}{|c|}{ Total CCA } & \multicolumn{3}{|c|}{ CAS scale } & \multicolumn{3}{|c|}{ CCB scale } \\
\hline & $N$ & $M$ & $S D$ & $N$ & $M$ & $S D$ & $N$ & $M$ & $S D$ \\
\hline Graduate & 58 & 5.73 & 0.64 & 59 & 6.18 & 0.42 & 58 & 5.29 & 1.04 \\
\hline Undergraduate & 82 & 5.59 & 0.72 & 85 & 5.99 & 0.49 & 82 & 5.19 & 1.18 \\
\hline$p$ value & & .219 & & & $.016^{*}$ & & & .596 & \\
\hline
\end{tabular}

I examined students' differences in cultural competency by program track groups using a one-way ANOVA. Findings showed a significant difference in CAS subscale scores by program track, $p<.032$ (see Table 17). Post hoc analysis used Fisher's least significant difference test $(p<.05)$ and found significant differences in the CAS subscale scores between the MSN ARNP students $(M=6.22)$ and RN-BSN Online students $(M=5.96)$, as well as between MSN ARNP students and Traditional BSN students $(M=5.97)$. 
Table 17

Means of Total Cultural Competency Assessment, Cultural Awareness and Sensitivity, and Cultural Competent Behaviors Subscales by Program track

\begin{tabular}{lrcccccccc}
\hline \multicolumn{1}{c}{ Variable } & \multicolumn{3}{c}{ Total CCA } & \multicolumn{3}{c}{ CAS subscale } & \multicolumn{3}{c}{ CCB subscale } \\
& $N$ & $M$ & $S D$ & $N$ & $M$ & $S D$ & $N$ & $M$ & $S D$ \\
\hline FEB BSN/MSN $^{\mathrm{a}}$ & 14 & 5.82 & 0.65 & 14 & 6.18 & 0.38 & 14 & 5.46 & 1.17 \\
RN-BSN online $^{\mathrm{b}}$ & 24 & 5.64 & 0.77 & 25 & 5.96 & 0.55 & 24 & 5.34 & 1.24 \\
Traditional BSN $^{\mathrm{c}}$ & 47 & 5.53 & 0.70 & 49 & 5.97 & 0.48 & 47 & 5.08 & 1.15 \\
MSN ARNP $^{\mathrm{d}}$ & 46 & 5.79 & 0.62 & 47 & 6.22 & 0.38 & 46 & 5.37 & 1.03 \\
MSN Anesthesia $^{\mathrm{e}}$ & 9 & 5.30 & 0.66 & 9 & 5.91 & 0.53 & 9 & 4.68 & 0.90 \\
$p$ value & & .148 & & & $.032^{*}$ & & & .340 & \\
\hline
\end{tabular}

Note. ${ }^{\text {a }}$ Foreign-educated physician/Bachelor of Science in Nursing and Foreign-education physician/Bachelor of Science in Nursing/Master of Science in Nursing combined; ${ }^{b}$ Registered nurse/Bachelor of Science in Nursing online combined; ${ }^{\mathrm{c}} \mathrm{BSN}=$ Bachelor of Science in Nursing; ${ }^{\mathrm{d}}$ Master of Science in Nursing/Advanced Registered Nurse Practitioners combined in Adult, Child, and Family tracks; ${ }^{\mathrm{e}} \mathrm{MSN}=$ Master of Science in Nursing; $\mathrm{CCA}=$ Cultural competency Assessment; CAS = Cultural Awareness and Sensitivity; $\mathrm{CCB}=$ Culturally Competent Behaviors; ${ }^{*} p<.05$.

For self-evaluated cultural competency, a cross-tabulation with a chi-square test indicated that there was no significant difference between MSN-level nursing education students and BSN-level students, $p=.136$. Of graduate students, $52 \%(n=32)$ evaluated themselves as very competent whereas $48 \%(n=39)$ of undergraduates did. A further cross-tabulation of self-evaluated cultural competency by program track was not significant, $p=.125$. Percentages of students who perceived themselves as "very competent," ranged from $37 \%(n=18)$ for traditional BSN students to $68 \%(n=17)$ of RN-BSN Online students. Hypothesis 1 was partially supported by these findings. The graduate student group (MSN) showed increased CAS scores compared to the undergraduate (BSN) student group. More in-depth analysis showed that the MSN ARNP program track group scored significantly higher on the CAS subscale than the RN-BSN Online program track group and the Traditional BSN program track group. Nevertheless, 
no significant differences were found among program track student groups on the total CCA scale or CCB subscale measures of competency. No significant differences were found on self-evaluated cultural competency by program level, $p=.237$ or program track, $p=.079$.

\section{Hypothesis 2: Cultural competency Levels and Program Standing (GPA).}

Research Question 2 sought differences between students' cultural competency levels and program standing student groups. Program standing was evaluated with self-reported GPAs. One-way ANOVAs resulted in significant differences found in total CCA scale scores and the CCB subscale between the higher program standing group (GPAs 3.5 to 4.0) and those in the lower program standing student group (GPAs 2.5 to 3.4; see Table 18). The higher GPA student group scored higher on both the total CCA scale and the CCB subscale than the lower GPA student group. There was no significant difference in self-evaluated cultural competence by program standing, $p=.609$. Forty eight percent ( $n$ $=57$ ) of students in the higher standing group perceived themselves as "very competent", similarly, $54 \%(n=14)$ of students in the lower standing group did.

Table 18

Cultural Competency of Nursing Students by Program Standing

\begin{tabular}{lrrrrrrrrr}
\hline \multicolumn{1}{c}{$\begin{array}{c}\text { Variable } \\
\begin{array}{c}\text { Grade-point } \\
\text { average }\end{array}\end{array}$} & $N$ & $M$ & $S D$ & $N$ & $M$ & $S D$ & $N$ & $M$ & $S D$ \\
\hline $2.5-3.4$ & 25 & 5.28 & 0.76 & 26 & 5.93 & 0.53 & 25 & 4.67 & 1.29 \\
$3.5-4.0$ & 115 & 5.73 & 0.65 & 118 & 6.10 & 0.45 & 115 & 5.36 & 1.04 \\
$p$ value & \multicolumn{3}{c}{$.003^{* *}$} & & & .09 & & & $.005^{* *}$ \\
\hline
\end{tabular}

Note. $\mathrm{CCA}=$ Cultural Competency Assessment; CAS = Cultural Awareness and Sensitivity; CCB = Cultural Competent Behaviors; ** $p<.01$. 


\section{Hypotheses 3 and 4: Personal and social factors and cultural competency.}

Research Question 3 inquired about relationships among independent variables and student groups by personal factors such as age, ethnicity/race, gender, and language used at home; and social factors such as previous diversity encounters and previous diversity training, with three measures of cultural competency variables (total CCA and CAS and CCB subscale scores). Two hypotheses were formulated for this research question, Hypothesis 3 and Hypothesis 4, as supported by the literature.

Hypothesis 3 predicted no significant associations of students' cultural competency levels (measured by the total CCA and the CAS and CCB subscales) and their personal factors by age, gender, ethnicity/race, and language spoken at home. However, Hypothesis 4 did predict significant associations of students' cultural competency levels by students' social factors of previous diversity encounters and previous diversity training.

Hypothesis 3 was assessed by Pearson's correlations of students' cultural competencies (measured by the total CCA scale and CAS and CCB subscales) with students' ages. Findings showed no significant associations of student age, $r=.14, .02, .17$, with the three measures of cultural competency (total CCA scale and CAS and CCB subscales) respectively (see Table 19). 
Table 19

Correlations of Total Cultural Competency Assessment, Cultural Awareness and Sensitivity, and Cultural Competent Behaviors Subscale Scores and Student Age

\begin{tabular}{lccc}
\hline & Total CCA & CAS & CCB \\
\hline Student age $(n)$ & 140 & 144 & 140 \\
Pearson's $r$ & .142 & .018 & .165 \\
$p$ value & .093 & .826 & .051 \\
\hline
\end{tabular}

Note $. \mathrm{CCA}=$ Cultural Competency Assessment; $\mathrm{CAS}=$ Cultural Awareness and Sensitivity; $\mathrm{CCB}=$ Cultural Competent Behaviors.

Hypothesis 3 was also assessed using one-way ANOVAs on students' cultural competencies (measured by total CCA and CAS and CCB subscales) by gender, ethnicity/race, and language spoken at home. One-way ANOVAs showed no significant differences in students' cultural competencies by gender (see Table 20).

Table 20

Total Cultural Competency Assessment, Cultural Awareness and Sensitivity, and Cultural Competent Behaviors By Gender

\begin{tabular}{|c|c|c|c|c|c|c|c|c|c|}
\hline \multirow{2}{*}{$\begin{array}{l}\text { Variable } \\
\text { Gender }\end{array}$} & \multicolumn{3}{|c|}{ Total CCA } & \multicolumn{3}{|c|}{ CAS scale } & \multicolumn{3}{|c|}{ CCB scale } \\
\hline & $N$ & $M$ & $S D$ & $N$ & $M$ & $S D$ & $N$ & $M$ & $S D$ \\
\hline Women & 116 & 5.65 & 0.69 & 119 & 6.08 & 0.48 & 116 & 5.22 & 1.12 \\
\hline Men & 24 & 5.65 & 0.67 & 25 & 6.01 & 0.44 & 24 & 5.31 & 1.13 \\
\hline$p$ value & & .999 & & & .537 & & & .725 & \\
\hline
\end{tabular}

Using one-way ANOVAs, I found no significant differences for any of the three measures of cultural competency by ethnic group (Hispanic, White/Caucasian/ European, Black/African American, Hispanic/White/Caucasian/European, Asian (several) and Multiethnic; see Table 21). 
Table 21

Cultural Competency Assessment, Cultural Awareness and Sensitivity, and Cultural Competent Behaviors by Ethnicity/Race

\begin{tabular}{lrrrrrrrrr}
\hline \multicolumn{1}{c}{ Variable } & \multicolumn{3}{c}{ Total CCA } & \multicolumn{3}{c}{ CAS scale } & \multicolumn{3}{c}{ CCB scale } \\
\multicolumn{1}{c}{ Ethnicity/race } & $N$ & $M$ & $S D$ & $N$ & $M$ & $S D$ & $N$ & $M$ & $S D$ \\
\hline Hispanic/White/Caucasian $^{c}$ & 15 & 5.86 & 0.60 & 15 & 6.32 & 0.43 & 15 & 5.41 & 1.02 \\
Hispanic/Latino $^{\mathrm{a}}$ & 70 & 5.75 & 0.69 & 72 & 6.08 & 0.47 & 70 & 5.40 & 1.13 \\
Asian $^{\mathrm{b}}$ & 4 & 5.60 & 0.15 & 4 & 5.84 & 0.36 & 4 & 5.36 & 0.30 \\
African American $_{\text {White/Caucasian/European }}$ & 21 & 5.56 & 0.59 & 22 & 6.08 & 0.45 & 21 & 5.06 & 1.06 \\
Multiethnic & 25 & 5.39 & 0.83 & 26 & 5.91 & 0.52 & 25 & 4.90 & 1.28 \\
$p$ value & 5 & 5.34 & 0.38 & 5 & 6.05 & 0.33 & 5 & 4.63 & 0.62 \\
\hline
\end{tabular}

Note. ${ }^{a}$ Hispanic/Latino includes: Mexican, Mexican American, Chicano, Cuban, Puerto Rican, others;

${ }^{\mathrm{b}}$ Asian includes Asian Indian, Chinese, Filipino, Japanese, Korean, Vietnamese, and other; CCA = Cultural Competency Assessment; $\mathrm{CAS}=$ Cultural Awareness and Sensitivity; $\mathrm{CCB}=$ Cultural Competent Behaviors.

And last, one-way ANOVAs did not find significant differences for any of the three measures of cultural competency (total CCA and CAS or CCB subscales) by language spoken at home (English, Spanish, and Others; see Table 22).

Table 22

Cultural Competency Assessment, Cultural Awareness and Sensitivity, and Cultural Competent Behaviors by Language

\begin{tabular}{|c|c|c|c|c|c|c|c|c|c|}
\hline \multirow{2}{*}{$\begin{array}{l}\text { Variable } \\
\text { Language }\end{array}$} & \multicolumn{3}{|c|}{ Total CCA } & \multicolumn{3}{|c|}{ CAS scale } & \multicolumn{3}{|c|}{ CCB scale } \\
\hline & $N$ & $M$ & $S D$ & $N$ & $M$ & $S D$ & $N$ & $M$ & $S D$ \\
\hline English & 79 & 5.56 & 0.69 & 82 & 6.04 & 0.47 & 79 & 5.10 & 1.10 \\
\hline Spanish & 53 & 5.79 & 0.71 & 54 & 6.13 & 0.48 & 53 & 5.44 & 1.19 \\
\hline Other & 8 & 5.57 & 0.30 & 8 & 5.97 & 0.28 & 8 & 5.17 & 0.69 \\
\hline$p$ value* & & .180 & & & .452 & & & .246 & \\
\hline
\end{tabular}


In summary, Hypothesis 3 was supported in that results showed no association of students' cultural competencies (measured by total CCA and CAS and CCB subscales) with age or differences by gender, ethnicity/race, or language spoken at home.

Hypothesis 4 predicted significant associations of students' cultural competency levels (measured by total CCA and CAS and CCB subscales) with the number of diversity encounters in their environment over the past 12 months, and previous participation in diversity training. Hypothesis 4 was first assessed by Pearson's correlations.

Previous diversity encounters. I used Pearson's correlations to test for relationships of students' total CCA and CAS and CCB subscale scores with the number of ethnic/racial groups encountered (out of a maximum of 7) and if each individual ethnic/racial group was encountered over the past 12 months. No correlations were significant (see Table 23). 
Table 23

Pearson's Correlations of Total Cultural Competency Assessment, Cultural Awareness

and Sensitivity, and Cultural Competent Behaviors Subscale Scores with Number of

Ethnic Groups Encountered and Each Type of Ethnic Group Encountered

\begin{tabular}{lccc}
\hline \multicolumn{1}{c}{ Ethnic group encountered } & Total CCA $(n=140)$ & CAS $(n=144)$ & CCB $(n=140)$ \\
\hline $\begin{array}{l}\text { Number of types of ethnic groups } \\
\text { encountered (maximum of 8) }\end{array}$ & .063 & .140 & .011 \\
Hispanic/Latino $^{\mathrm{a}}$ & .032 & -.028 & .039 \\
White/Caucasian/European $^{\text {African American/Black }}{ }^{\mathrm{b}}$ & -.017 & .033 & -.023 \\
American Indian/Alaskan Native $^{\text {Asian }}{ }^{\mathrm{c}}$ & -.057 & -.038 & -.045 \\
Hawaiian/Pacific Islander & .133 & .149 & .098 \\
Arab/Middle Eastern $^{\text {Other }}$ & -.033 & .050 & -.067 \\
\hline
\end{tabular}

Note. ${ }^{a}$ Hispanic includes Latino, Mexican, Mexican American, Chicano, Cuban, Puerto Rican, and others;

${ }^{\mathrm{b}}$ African American/Black included Caribbean, Haitian, and Jamaican; ${ }^{\mathrm{C}}$ Asian included Asian Indian, Chinese, Filipino, Japanese, Korean, and Vietnamese; ${ }^{\mathrm{d}}$ Other includes Ukrainian, Serbian, and Brazilian; $\mathrm{CCA}=$ Cultural Competency Assessment; $\mathrm{CAS}=$ Cultural Awareness and Sensitivity; $\mathrm{CCB}=$ Cultural Competent Behaviors.

Using Pearson's correlations, I also tested for relationships of students' cultural competencies (measured by total CCA and CAS and CCB subscales) with the number of special populations encountered (of a maximum of 8) over the past 12 months in their environments (see Table 24). The number of special populations encountered was not positively correlated with any of the measures of cultural competency. Significant inverse relationships resulted in two of the measures of cultural competency-the total CCA scale, $r=-.18$, and the CAS subscale, $r=-.29$, with those who had encountered homeless/housing-insecure clients. A significant positive relationship was also found between CAS and those who had encountered clients with different religious/spiritual 
backgrounds in their environment over the past 12 months, $r=.20$. In other words, students who had encountered homeless or housing-insecure clients scored lower on the CAS subscale as well as on the total CCA scale score. Also, those who encountered clients from different religions or spiritual backgrounds scored higher on the CAS subscale.

Table 24

Pearson's Correlations of Total Cultural Competency Assessment, Cultural Awareness and Sensitivity, and Cultural Competent Behaviors Subscales with Number of Special Populations Encountered and Each Special Population Encountered

\begin{tabular}{lccc}
\hline \multicolumn{1}{c}{ Special populations encountered } & Total CCA $(n=140)$ & CAS $(n=144)$ & CCB $(n=140)$ \\
\hline $\begin{array}{l}\text { Number of special populations } \\
\text { encountered (maximum of 8) }\end{array}$ & .058 & .061 & .035 \\
Mentally/emotionally ill & .076 & -.131 & .143 \\
Physically challenged/disabled & .033 & -.042 & .049 \\
Homeless/housing insecure & $-.176^{*}$ & $-.288^{* *}$ & -.092 \\
Substance abusers/alcoholics & -.094 & -.121 & -.062 \\
Lesbian/bisexual/gay/transgendered & -.047 & -.112 & -.019 \\
Different religious/spiritual & .101 & $.203 *$ & .031 \\
No special populations encountered & -.030 & .051 & -.060 \\
Other & -.069 & -.083 & -.050 \\
\hline $\begin{array}{l}\text { Note. CCA }=\text { Cultural Competency Assessment; CAS }=\text { Cultural Awareness and Sensitivity; CCB }= \\
\text { Cultural Competent Behaviors; }{ }^{*} p<.05, * * p<.01 .\end{array}$ & &
\end{tabular}

Previous diversity training. Differences on total CCA and CAS and CCB subscale scores were found between those who had attended previous diversity training and those that responded they had not. This was evaluated by a one-way ANOVA and significant differences were found on all three measures of cultural competency: the total CCA, $p<.005$; the CAS subscale, $p<.035$; and the CCB subscale, $p<.012$ (see Table 
25). Those who had participated in previous diversity training scored higher on all three measures of cultural competency.

Table 25

Total Cultural Competency Assessment, Cultural Awareness and Sensitivity, and Cultural Competent Behaviors Subscale Scores by Previous Diversity Training

\begin{tabular}{lccccccccc}
\hline \multicolumn{1}{c}{$\begin{array}{c}\text { Variable } \\
\begin{array}{c}\text { Previous } \\
\text { diversity training }\end{array}\end{array}$} & $N$ & $M$ & $S D$ & $N$ & $M$ & $S D$ & $N$ & $M$ & $S D$ \\
\hline Yes & 91 & 5.76 & 0.63 & 92 & 6.13 & 0.47 & 91 & 5.41 & 1.03 \\
No & 49 & 5.43 & 0.73 & 52. & 5.96 & 0.46 & 49 & 4.91 & 1.21 \\
$p$ value* & & $.005^{* *}$ & & & $.035^{*}$ & & & $.012^{*}$ & \\
\hline
\end{tabular}

Note. $\mathrm{CCA}=$ Cultural Competency Assessment; $\mathrm{CAS}=$ Cultural Awareness and Sensitivity; $\mathrm{CCB}=$ Cultural Competent Behaviors; ${ }^{*} p<.05,{ }^{* *} p<.01$.

I performed further testing and used Pearson's correlations to evaluate for associations of cultural competency, measured by the total CCA scale and the CAS and CCB subscales, with the number of types of diversity training attended (out of 6), the total number of times students ever participated in diversity training in all categories, and if each type of diversity training was taken. Because the total number of times trained in all categories was a count and had a wide range ( 0 to 60 ), I applied a square-root transformation to normalize the variable; I also used this new variable in the correlation analysis.

The higher the number of types of training attended (out of 6), the higher were all measures of cultural competency $(p<.05$; see Table 26). The higher the total number of times trained in all categories, the higher were all three measures of cultural competency $(p<.05)$ for the raw count and the transformed variable. Finally, the attended diversity training group was significantly associated with the continuing education type of training 
and was significantly associated with higher cultural competency by total CCA scale scores, $r=.23$; CAS subscale scores, $r=.19$; and CCB subscale scores, $r=.20$.

Table 26

Pearson's Correlations of Total Cultural Competency Assessment, Cultural Awareness and Sensitivity, and Cultural Competent Behaviors Subscale Scores with Diversity Training Measures

\begin{tabular}{lcll}
\hline \multicolumn{1}{c}{ Measures } & Total CCA & CAS & CCB \\
\hline $\begin{array}{l}\text { Diversity training (yes) } \\
\begin{array}{l}\text { Number of types of diversity } \\
\text { training }\end{array}\end{array}$ & $.234^{* *}$ & $.176^{*}$ & $.212^{*}$ \\
$\begin{array}{l}\text { Total number of times trained in } \\
\text { all categories }\end{array}$ & $.210^{*}$ & $.223^{* *}$ & $.205^{*}$ \\
$\begin{array}{l}\text { Square root of total number of } \\
\text { times trained in all categories }\end{array}$ & $.246^{* *}$ & $.213^{*}$ & $.168^{*}$ \\
$\begin{array}{l}\text { Separate college courses for } \\
\text { credit }\end{array}$ & .060 & $.248^{* *}$ & $.198^{*}$ \\
$\begin{array}{l}\text { Content covered in a course } \\
\text { Professional conference/seminar }\end{array}$ & .124 & -.016 & .079 \\
$\begin{array}{l}\text { Employer-sponsored program } \\
\text { Online (computer-assisted }\end{array}$ & .135 & .150 & .090 \\
education) & .147 & .046 & .144 \\
Continuing education & $.231^{* *}$ & $.171^{*}$ & .045 \\
\hline
\end{tabular}

Note. $\mathrm{CCA}=$ Cultural Competency Assessment; $\mathrm{CAS}=$ Cultural Awareness and Sensitivity; $\mathrm{CCB}=$ Cultural Competent Behaviors; ${ }^{*} p<.05,{ }^{* *} p<.01$.

Results of these tests supported Hypothesis 4. Students who had attended a greater number of types of previous diversity training, who were trained in diversity in all categories a greater number of times, and who had attended continuing-education diversity training had significantly higher total CCA scale scores, and CAS and CCB subscale scores. 


\section{Multiple linear regression analysis of Hypotheses 3 and 4. Pearson's}

correlations initially tested the relationships of factors of: personal, educational, and previous diversity training with the total CCA scale and CAS and CCB subscales. For the personal predictor of White/non-Hispanic, I found significant associations among the White/non-Hispanic student group and total CCA scores, $r=-.18, p=.036$, and between the Hispanic/White/Caucasian/European student group and CAS subscale scores, $r=.18$, $p=.030$ (see Table 27). White/non-Hispanic students scored lower on the total CCA scale whereas Hispanic/White/European students scored higher on the CAS subscale. As seen earlier, age, gender and language spoken at home variables were not related to any of the measures of cultural competency.

As seen earlier, for educational factors, the graduate-level student group scored higher on the CAS subscale, and the higher GPA student group scored higher on the total CCA scale as well as the CCB subscale. Last, those who attended more types of diversity training scored higher on all three measures of cultural competency levels (the total CCA and CAS and CCB subscales; see Table 28). 
Table 27

Pearson's Correlations of Total Cultural Competency Assessment, Cultural Awareness and Sensitivity, and Cultural Competent Behaviors Subscale Scores with Personal

Factors

\begin{tabular}{lccc}
\hline \multicolumn{1}{c}{ Factors } & Total CCA & CAS & CCB \\
\hline Age & .142 & .018 & .165 \\
White/non-Hispanic & $-.178^{*}$ & -.162 & -.140 \\
Black/African American $^{\mathrm{a}}$ & -.056 & .010 & -.064 \\
Asian $^{\mathrm{b}}$ & -.012 & -.082 & .020 \\
Hispanic/White/European $_{\text {Multiethnic }}$ & .109 & $.180^{*}$ & .055 \\
Men & -.087 & -.005 & -.105 \\
Spanish spoken at home & .000 & -.052 & .030 \\
Other language spoken at home & .157 & .100 & .142 \\
\hline
\end{tabular}

Note. ${ }^{a}$ Black/African American $=$ African American, Black, Caribbean, Haitian, Jamaican; ${ }^{\mathrm{b}}$ Asian includes Asian Indian, Chinese, Filipino, Japanese, Korean, Vietnamese, and other; CCA = Cultural Competency Assessment; $\mathrm{CAS}=$ Cultural Awareness and Sensitivity; $\mathrm{CCB}=$ Cultural Competent Behaviors; ${ }^{*} p<.05,{ }^{* *} p<.01$.

\section{Table 28}

Pearson's Correlations of Total Cultural Competency Assessment, Cultural Awareness and Sensitivity, and Cultural Competent Behaviors Subscale Scores with Education, Diversity Training, and Encounters

\begin{tabular}{lccc}
\hline \multicolumn{1}{c}{ Factors } & Total CCA $(n=140)$ & CAS $(n=144)$ & CCB $(n=140)$ \\
\hline Graduate level & .104 & $.207^{*}$ & .041 \\
$\begin{array}{l}\text { Higher grade-point averages }(3.5- \\
4.0)\end{array}$ & $.249^{* *}$ & .141 & $.237^{* *}$ \\
$\begin{array}{l}\text { Number of types of diversity } \\
\text { training (maximum of 6) }\end{array}$ & $.243^{* *}$ & $.223^{* *}$ & $.205^{*}$ \\
$\begin{array}{l}\text { Number of types of ethnic groups } \\
\text { encountered (maximum of 8) }\end{array}$ & .063 & .140 & .011 \\
$\begin{array}{l}\text { Number of special populations } \\
\text { encountered (maximum of 7) }\end{array}$ & .058 & .061 & .035 \\
\hline
\end{tabular}

Note. $\mathrm{CCA}=$ Cultural Competency Assessment; $\mathrm{CAS}=$ Cultural Awareness and Sensitivity; $\mathrm{CCB}=$ Cultural Competent Behaviors; ${ }^{*} p<.05,{ }^{* *} p<.01$. 
Multiple regression analysis. Multiple regression analysis predicted each of the three scales - total CCA and CAS and CCB_-from four blocks of independent variables: personal, educational, diversity training, and diversities encountered. Predictors entered into the first block included age, ethnicity (White/non-Hispanic, Black/African American, Asian, Hispanic/White/European, and Multiethnic, with Hispanic as the base), male (gender), and language spoken at home (Spanish, other language, with English as base). The second block of predictors entered graduate-program level and higher GPA (program standing). The third block entered the number of diversity training types (maximum $=6$ ), and the fourth block of predictors entered the number of ethnic groups encountered and the number of special populations encountered.

The overall regression model for students' cultural competency, measured by the total CCA scale was significant $\left(R^{2}=.18, p=.027\right.$; see Table 29$)$. In other words, $18 \%$ of the variability of the total CCA scale was explained by this set of predictors. In this model, the first block of personal factors was not significant, $p=299$. The second block of educational variables explained an additional $5.9 \%$ of variability, $p=.015$, and, higher GPAs (3.5-4.0) was a significant predictor of total CCA scale $(\beta=.22, p=.012)$. The third block of diversity training explained an additional $4.3 \%$ of the variability of total CCA scale, and was found to be significant, $p=.011$. The number of types of diversity training $(\beta=.23, p=.025)$ was also significant. The fourth and final block added the predictors of number of types of ethnic/racial and special populations encountered, but was not significant, $p=.898$. Controlling for all other predictors, higher total CCA scale scores were associated with higher GPAs and a greater number of diversity training types taken. 
Table 29

Model Summary of Regression of Total Cultural Competency Assessment on Personal, Educational, Diversity Training, and Diversities Encountered

\begin{tabular}{|c|c|c|c|c|c|}
\hline Predictors & $\beta$ & $p$ & $R^{2}$ & Change in $R^{2}$ & $p$ value \\
\hline Block 1-Personal & & & .077 & .077 & .299 \\
\hline Age & .06 & .491 & & & \\
\hline White/non-Hispanic & $-.21^{*}$ & .047 & & & \\
\hline African American/Black ${ }^{\mathrm{a}}$ & -.07 & .489 & & & \\
\hline $\operatorname{Asian}^{\mathrm{b}}$ & -.01 & .917 & & & \\
\hline Hispanic/White/European & .01 & .899 & & & \\
\hline Multiethnic & -.16 & .085 & & & \\
\hline Spanish spoken at home & .02 & .885 & & & \\
\hline $\begin{array}{l}\text { Other language spoken at } \\
\text { home }\end{array}$ & -.03 & .807 & & & \\
\hline Men & -.06 & .495 & & & \\
\hline Block 2-Educational & & & .136 & $.059 *$ & .015 \\
\hline Graduate program level & -.03 & .776 & & & \\
\hline $\begin{array}{l}\text { Higher grade-point } \\
\text { averages }(3.5-4.0)\end{array}$ & $.22 *$ & .012 & & & \\
\hline Block 3-Diversity training & & & .178 & $.043 *$ & .011 \\
\hline $\begin{array}{l}\text { Number of types of } \\
\text { diversity training }\end{array}$ & $.23 *$ & 0.25 & & & \\
\hline \multicolumn{3}{|c|}{ Block 4-Diversities encountered } & .180 & .001 & .898 \\
\hline $\begin{array}{l}\text { Number of types of } \\
\text { ethnic groups } \\
\text { encountered (max of } 8)\end{array}$ & .02 & .870 & & & \\
\hline $\begin{array}{l}\text { Number of special } \\
\text { populations encountered } \\
\text { (max of } 7 \text { ) }\end{array}$ & .03 & .737 & & & \\
\hline
\end{tabular}

The second regression was performed with the CAS subscale as the dependent variable and the same predictors as in the first analysis (see Table 30). Results of the second regression model showed significant overall regression, $\left(R^{2}=.16, p=.047\right)$. In 
this model, the second block was significant, $p=.011$, and explained $6.2 \%$ additional variability of the CAS subscale. However, neither graduate program nor higher GPA reached significance. The third block added the number of diversity training types attended, was found to be significant, $p=.05$, and explained $2.5 \%$ additional variability of the CAS subscale. The fourth and final block added predictors of ethnic/racial and special population diversity encounters, but was not found to be significant, $p=.463$, explaining an additional $1 \%$ of variability of the CAS subscale (see Table 30 ). In summary, no individual predictors were significant in the multiple regression analysis on the CAS subscale, although the blocks of educational predictors and the number of diversity training types attended explained significant additional variability $(8.7 \%)$ of the CAS subscale. 
Table 30

Model Summary of Regression of Cultural Awareness and Sensitivity Subscale on Personal, Educational, Diversity Training, and Diversities Encountered

\begin{tabular}{|c|c|c|c|c|c|}
\hline Predictors & $\beta$ & $p$ & $R^{2}$ & Change in $R^{2}$ & $p$ value \\
\hline Block 1-Personal & & & .065 & .065 & .417 \\
\hline Age & -.106 & .253 & & & \\
\hline White/non-Hispanic & -.185 & .082 & & & \\
\hline African American/Black ${ }^{\mathrm{a}}$ & .021 & .840 & & & \\
\hline $\operatorname{Asian}^{\mathrm{b}}$ & -.108 & .378 & & & \\
\hline Hispanic/White/European & .105 & .246 & & & \\
\hline Multiethnic & -.057 & .522 & & & \\
\hline Spanish spoken at home & .071 & .531 & & & \\
\hline $\begin{array}{l}\text { Other language spoken at } \\
\text { home }\end{array}$ & .069 & .569 & & & \\
\hline Men & -.086 & .314 & & & \\
\hline Block 2-Educational & & & .127 & $.062 *$ & .011 \\
\hline Graduate program level & .177 & .064 & & & \\
\hline $\begin{array}{l}\text { Higher grade-point } \\
\text { averages }(3.5-4.0)\end{array}$ & .074 & .392 & & & \\
\hline Block 3-Diversity training & & & .152 & $.025^{*}$ & .050 \\
\hline $\begin{array}{l}\text { Number of types of } \\
\text { diversity training }\end{array}$ & .164 & .103 & & & \\
\hline \multicolumn{2}{|c|}{ Block 4-Diversity encountered } & & .162 & .010 & .463 \\
\hline $\begin{array}{l}\text { Number of types of } \\
\text { ethnic groups } \\
\text { encountered (max of } 8 \text { ) }\end{array}$ & .123 & .216 & & & \\
\hline $\begin{array}{l}\text { Number of special } \\
\text { populations encountered } \\
\text { (max of } 7 \text { ) }\end{array}$ & -.049 & .628 & & & \\
\hline
\end{tabular}

Note. ${ }^{a}$ African American/Black included Caribbean, Haitian, Jamaican; ${ }^{b}$ Asian included Asian Indian, Chinese, Filipino, Japanese, Korean, Vietnamese; ${ }^{*} p<.05,{ }^{* *} p<.01 . R^{2}=.16, F_{(14,129)}=1.79, p=.047$.

A third regression model for the $\mathrm{CCB}$ subscale did not reach significance $\left(R^{2}=.16, p=.064 ;\right.$ see Table 31$)$. However, examination of the blocks of variables entered was completed to describe the model to complete analysis of Hypotheses 3 and 4 . 
The second block of educational variables explained an additional $5.5 \%$ of variability, $p=.021$, and higher GPAs (3.5-4.0), was a predictor on the CCB subscale $(\beta=.23$, $p=.009$ ). The third block of diversity training explained an additional $3.5 \%$ of the variability of the total CCA scale, $p=.011$. The number of types of diversity training $(\beta=.16, p=.033)$ was a positive predictor of the CCB subscale. In summary, the multiple regression analysis of the CCB subscale is similar to that of the total CCA scale except that significance was not reached. 
Table 31

Model Summary of Regression of Cultural Competent Behaviors Subscale on Personal, Educational, Diversity Training, and Diversities Encountered

\begin{tabular}{|c|c|c|c|c|c|}
\hline Predictors & $\beta$ & $p$ & $R^{2}$ & Change in $R^{2}$ & $p$ value \\
\hline Block 1-Personal & & & .069 & .069 & .391 \\
\hline Age & .117 & .217 & & & \\
\hline White/non-Hispanic & -.175 & .105 & & & \\
\hline African American/Black ${ }^{\mathrm{a}}$ & -.082 & .445 & & & \\
\hline $\operatorname{Asian}^{\mathrm{b}}$ & .034 & .785 & & & \\
\hline Hispanic/White/European & -.030 & .740 & & & \\
\hline Multiethnic & -.168 & .070 & & & \\
\hline Spanish spoken at home & -.019 & .870 & & & \\
\hline $\begin{array}{l}\text { Other language spoken at } \\
\text { home }\end{array}$ & -.076 & .539 & & & \\
\hline Men & -.020 & .816 & & & \\
\hline Block 2-Educational & & & .123 & $.055^{*}$ & .021 \\
\hline Graduate program level & -.105 & .275 & & & \\
\hline $\begin{array}{l}\text { Higher grade-point } \\
\text { averages }(3.5-4.0)\end{array}$ & $.231 * *$ & .009 & & & \\
\hline Block 3-Diversity training & & & .158 & $.035^{*}$ & .024 \\
\hline $\begin{array}{l}\text { Number of types of } \\
\text { diversity training }\end{array}$ & $.158^{*}$ & .033 & & & \\
\hline Block 4-Diversity encounter & & & .160 & .001 & .898 \\
\hline $\begin{array}{l}\text { Number of types of } \\
\text { ethnic groups } \\
\text { encountered (maximum } \\
\text { of } 8 \text { ) }\end{array}$ & -.038 & .704 & & & \\
\hline $\begin{array}{l}\text { Number of special } \\
\text { populations encountered } \\
\text { (maximum of } 7 \text { ) }\end{array}$ & .040 & .696 & & & \\
\hline
\end{tabular}

To summarize the multiple regression analyses of Hypotheses 3 and 4, the blocks of educational predictors and number of types of diversity training were significant in predicting all three cultural competency scales. For the total CCA scale, controlling for 
all other predictors, higher scores were associated with higher GPAs and a greater number of diversity-training types taken. However, for the two subscales, the results were weaker.

\section{Summary}

Hypothesis 1 was partially supported. The graduate student group (MSN) showed increased CAS scores compared to the undergraduate (BSN) student group. More indepth analysis showed that the MSN ARNP program track group scored significantly higher on the CAS subscale than the RN-BSN Online program track group and the Traditional BSN program track group. There were no significant differences for total CCA scale or CCB subscale.

Hypothesis 2 was partially supported. The higher GPA student group (3.5-4.0) scored higher on both the total CCA scale and the CCB subscale than the lower GPA student group, but there was no difference for the CAS subscale.

Hypothesis 3 was supported using univariate analysis. The results showed no associations of students' cultural competencies (measured by total CCA and CAS and CCB subscales) with age or differences by gender, ethnicity/race, or language spoken at home.

Hypothesis 4 was partially supported using univariate analysis. Students who had attended a greater number of types of previous diversity training, who were trained in diversity in all categories a greater number of times, and who had attended continuingeducation diversity training had significantly higher total CCA scale scores, and CAS and CCB subscale scores. However, no support was found for relating students' cultural 
competency scores and the number and types of diverse ethnic/racial and specialpopulation encounters.

Hypotheses 3 and 4 were partially supported by multivariate analysis. For the multiple regression analyses, the blocks of educational predictors and number of types of diversity training were significant in predicting all three cultural competency scales. For the total CCA scale, controlling for all other predictors, higher scores were associated with higher GPAs and a greater number of diversity training types taken.

Findings from this student sample supported the hypotheses with the exception of the theoretical expectation of the relationships of number of diverse ethnic/racial and special populations encountered with students' cultural competency for the three measures. With that said, more research is recommended to explore students' lived experiences with ethnic/racial and special population groups encountered in their healthcare and workplace environments. 


\section{Chapter 5}

Discussion 


\section{CHAPTER 5}

\section{DISCUSSION}

This chapter presents a discussion of the study findings, conclusions, implications, limitations, and recommendations for future research. To recapitulate, the goal of the study was to evaluate differences between undergraduate (BSN) and graduate (MSN) nursing students' cultural competency levels, as well as determine any associations of student cultural competency with personal, educational, and social factors.

\section{Characteristics}

Initial descriptive analysis found this sample to be atypical of many nursing student groups in previous cultural competency literature. Student mean age was 32.6 years and the sample was predominantly female $(83 \%)$. These two characteristics were the only attributes similar to previous research. This student sample was unique from that portrayed in the literature by ethnicity/race and language spoken at home. Regrouping of students was necessary to represent the large percentage that identified with two or more ethnicities/races. Fifty percent were Hispanic/Latino whereas another 13.5\% were Multiethnic or Hispanic/Latino/White/Caucasian/European. Few studies resembled this composition, although Hagman (2006) reported creation of a multiethnic-grouping variable to represent the $21 \%$ in that study who had identified themselves as multiethnic.

Another unique attribute was the fact that more than $43 \%$ of this sample spoke Spanish or another language at home. The literature often reported primarily Englishspeaking nursing-student samples (Alpers \& Zoucha, 1996; Amerson, 2010; Anderson, 2004; Baldonado et al., 1998; Benkert et al., 2005; Bond, Kardong-Edgren, \& Jones, 2001; Eliason \& Raheim, 2000; Fitzgerald, Cronin, \& Campinha-Bacote', 2007). Some 
researchers excluded ethnicity and language from analyses, believing that a lack of variability existed in their studies (Benkert et al., 2011).

\section{Educational Factors, Differences between Nursing-Student Groups}

Findings of differences between nursing students' cultural competency levels by educational/program level corroborated previous reports (Bond et al., 2001; Brathwaite, 2005, 2006; Doorenbos \& Schim, 2004). In past research, significant differences were found in transcultural self-efficacy between 1st- and 4th-semester associate degree in nursing students (Jeffreys \& Dogan, 2012). Of note, associate degree graduates comprised $17.4 \%$ of the RN-BSN program group in the present sample. The present findings lend support to Starr and Wallace's (2009) report of significant differences in cultural competency among nursing students by educational level. The present study found differences between undergraduate (BSN) and graduate (MSN) nursing students in cultural awareness and sensitivity.

Contradictory findings were reported in earlier studies, indicating that BSN students scored higher than graduate students on alternate cultural competency measures (Bond et al, 2001; Campinha-Bacote', 2007; Hagman, 2006; Krainovich-Miller et al., 2008). One study reported that doctoral students scored lower on cultural competency than BSN and MSN students (Hagman, 2006). Researchers observed that higher levels of education had created heightened awareness and realization of possessing little cultural knowledge (Campinha-Bacote, 2007; Hagman, 2006; Krainovich-Miller et al., 2008); however, such finding could not be confirmed in the present study. Doctoral students were excluded from the present sample because of their very limited number compared to 
BSN and MSN students, and that decision was further supported by findings in earlier studies (ref).

MSN ARNP program track students were found to be more culturally aware and sensitive than the traditional BSN and RN-BSN online students; however, these two groups of students did not differ in culturally competent behaviors, and overall cultural competency levels. Also, differences in all three cultural competency measures (overall CCA, CAS and CCB subscales) were not seen between students in Anesthesiology (MSN) and FEP (FEP BSN/MSN) program tracks. There is reason to believe that the direction of the present findings might have been influenced by the limited number of respondents from the Anesthesiology and FEP BSN/MSN groups. Also, the graduate (MSN) ARNP curriculum requires a stand-alone culture course (NGR 5131), whereas undergraduate (RN-BSN Online and Traditional BSN) curricula thread cultural content throughout the program, and there is no required stand-alone culture course.

Although there is support in the literature for differences in perception of a culturally competent curriculum, evaluated with BSN, MSN, and DNP/PhD students, neither specialty tracks nor program standing have been evaluated, making the present study unique and valuable (A. M. Brennan \& Cotter, 2008; Cuellar et al., 2008). No differences were found in perceived cultural competency as measured by one five-point Likert scale item, among students in different program levels or program tracks. Of note, more than $90 \%$ of students rated themselves culturally competent, demonstrating very little variance. None of the students in this sample evaluated themselves as "somewhat incompetent" or "very incompetent," suggesting that this student sample was extraordinary in cultural competency levels. 
Students were evaluated for differences in cultural competency levels by program standing, determined by self-reported GPAs. Differences between students by program standing (GPA) were found in overall culturally competent assessment and culturally competent behaviors, but not in cultural awareness and sensitivity, in favor of students with higher self-reported GPAs (3.5-4.0). This is in contrast to the depicted differences for cultural awareness and sensitivity found between students by program level and program track comparisons. This is a puzzling finding because GPA is a representation of academic standing, and a significant difference between program standing groups (GPAs) on cultural awareness (knowledge) and sensitivity is expected in the cognitive domain. Also surprising was the finding with respect to program level and program track (CAS subscale only) that differed from program standing (total CCA and CCB subscale) results. Furthermore, there were no differences found between self-evaluated cultural competency groups by program standing (GPAs) and by program level groups. It should be mentioned that nearly all graduate-level (MSN) students and most undergraduate students categorized themselves at higher program standing, with a total sample average GPA of $3.82(S D=0.4)$. Students also perceived themselves, overall, as culturally competent. Of note, graduate-level programs require GPAs greater than 3.00 and undergraduate (BSN) programs require a minimum GPA of 3.00 to maintain enrollment.

Program standing (GPA) is hardly addressed in the literature with respect to evaluation of nursing students' cultural competency levels. Felder (1990) included GPA in an evaluation of undergraduate freshman, seniors, and associate's degree nursing students' cultural competency. Reported findings showed the lowest GPA, 1.9, was from the associate degree group and the highest GPA, 3.9, was from the baccalaureate-level 
student group (Felder, 1990). Felder (1990) found that most students reported GPAs from 3.0 to 4.0 , and this was similar to the present samples' self-reported program standings, with most in the higher GPA 3.5-4.0 group. Students' GPAs are sensitive information, according to the Family Educational Rights and Privacy Act (FERPA), preventing researchers from obtaining student's individual transcript GPA data. This evaluation relied on self-reported GPAs and may have a degree of inaccuracy or error due to students' calculations or memories. As with any self-reported data, caution should be taken when making generalizations.

\section{Personal Factors}

Bednarz et al. (2010) evaluated nursing students' cultural competency with the CCA and used regression to evaluate nurses' ages as a predictor. Researchers in that study found that age explained a significant amount of variability in CAS subscale results (Bednarz et al., 2010). Kardong-Edgren et al. (2010) believed younger students lacked "life experiences" compared with older students, which supported differences found on the IAPCC- $\mathrm{R}^{\circledR}$ measure of cultural competency. The present study fails to corroborate those previous findings. Of note, personal attributes of students in this study included age, gender, ethnicity/race, and language spoken at home. Pearson's correlations revealed no relationship of students' ages with their cultural competency levels. In all three regression equations the set of personal attributes of age, gender, ethnicity/race, and language at home, did not explain significant amounts of variability of the total CCA, the CAS and CCB subscales. The same personal attributes were also evaluated by one-way ANOVA with the three measures of cultural competency. Similarly, no significant differences were found by gender, ethnicity/race, or language spoken at home $(p \mathrm{~s}>.05)$. Therefore, 
these findings lend support for the null hypotheses of personal attributes having no association with cultural competency levels.

\section{Social Factors}

Antithetical to Kolb's (1984) experiential learning theory, Benner's (1982) novice-to-expert theory, and Ryan and Twibell's (2002) matrix for growth through immersions, the present findings lend only minimal support for the expected association of increased numbers of diversity client encounters with cultural competency levels. Licensed to practice as registered nurses, RN-BSN Online students and most graduatelevel (MSN) students would have more encounters with diverse clients in healthcare or workplace environments than the traditional BSN students, who were just preparing to become RNs. Furthermore, there was no relationship shown between the students' numbers of ethnic/racial groups encountered and the three measures of cultural competency.

Pearson's correlations were similarly used to test the relationship between the competency measures and the number of special populations encountered, which included mentally/emotionally ill, physically challenged/disabled, substance abusers/alcoholics, homeless/housing insecure, sexual orientation (gay, lesbian, bisexual, and transgendered), and different religious and spiritual views. Although the literature provided strong support for higher numbers of diverse encounters to be associated with students' cultural competency levels (Campinha-Bacote, 2007; Leininger, 1995), this was not reflected in the present study. Of note, the evaluation of special populations is rarely addressed in studies of nursing students' cultural competency levels (Eliason \& Raheim, 2000; S. M. Schim, personal communication, October 25, 2011). 
Of note, there was an inverse relationship shown in this study, demonstrating that students who had encountered more homeless/housing-insecure clients scored significantly lower on overall CCA, $p<.05$, and on CAS mean scores, $p<.01$. However, a positive relationship was found among students who had encountered more clients of different religious/spiritual views with higher level of cultural awareness and sensitivity, $p<.05$. This finding suggests that increased exposure to cultural diversity is likely to increase students' CAS. A qualitative inquiry merits consideration to explore students' lived experiences related to encounters with homeless/housing insecure clients. Previous research that evaluated cultural competency with the CCA instrument had created a new variable by counting the types of ethnic/racial and special populations encountered; this variable was similarly created in the present analysis and evaluated in the same way (Doorenbos \& Schim 2004; Schim, Doorenbos, \& Borse, 2005; Schim et al., 2006).

Schim and Doorenbus (2004, p.31) reported that the number of ethnic/racial and special populations encountered did not have a significant relationship to healthcare workers' cultural competency. Of note, the sample for the present study was composed of nursing students in different program types and program standing, compared to the sample in the previous studies, composed of different types of healthcare and/or hospice workers. As indicated by Starr \& Wallace (2009), research with the CCA instrument used with a similar sample of nursing students has been rarely done. In comparison, Jones et al. (2004) reported a lack of association of participants' cultural attitudes and cultural self-efficacy, despite their reported $71 \%$ to $100 \%$ of their time spent with diverse clients. Furthermore, research that reported significant associations with previous 
diversity encounters and students' cultural competency levels included a sample that had previous international travel as a factor or measure of encounters and evaluated cultural competency with a different measure, the IAPCC- ${ }^{\circledR}$ scale (Kardong-Edgren \& Campinha-Bacote, 2008)

In the present study, the last evaluation of the number of diverse clients encountered was conducted in three regression models. In all three regression analyses the number of ethnic/racial clients or the number of special populations encountered did not explain a significant amount of variability in any of the three cultural competency measures (total CCA; CAS and CCB subscales) as the dependent variables.

Background literature often used the concepts of encounters interchangeably with experiences, exposures, immersions, and even service learning in the evaluation of nursing students' cultural competency (Amerson, 2010; Bond \& Jones, 1994; Caffrey et al., 2005; Carpio \& Majumdar, 1993; Green et al., 2011; Jones, Cason, \& Bond, 2004; Kardong-Edgren \& Campinha-Bacote, 2008; Kollar \& Ailinger, 2002; Larson et al., 2010; Reeves \& Fogg, 2006; Riner \& Becklenberg, 2011). The association of previous diversity training with cultural competency levels was expected and supported by several researchers' findings (Alpers \& Zoucha, 1996; Bassi, 2011; Bond et al., 2001; Brathwaite, 2005; Hagman, 2006; Hughes \& Hood, 2007; Jones et al., 2004; Kardong-Edgren \& Campinha-Bacote, 2008).

Starr and Wallace (2009) reported that"participation in professional conferences, seminars and online courses" diversity-training methods were significantly associated with participants' cultural awareness and sensitivity (CAS) and culturally competent behaviors $(\mathrm{CCB})$ and overall cultural competency $(\mathrm{CCA})$. They found that the most 
attended of all types of diversity training listed were professional conferences and seminars. In the present study, diversity training was evaluated in two ways but also required the creation of variables the number of types of diversity training and the total number of times trained in all categories, following a "yes" response to the use of diversity training. A square-root transformation variable was also created and called the "square root of total number of times trained in all categories."

In the present sample, $63.9 \%(n=92)$ had attended diversity training and the type of training attended most was "content within a college course for credit" $(40.3 \%)$. Results of a one-way ANOVA between diversity training student groups ("yes" and "no") and cultural competency measures showed a significant association among all three measures of cultural competency: the total CCA and CAS and CCB subscales $(p=.005$, $p=.035$, and $p=.012$, respectively). Further analysis indicated that although "content within a college course for credit" was the type of diversity training attended most, the type of training significantly associated with all three measures of students' cultural competency levels was "continuing education" (total CCA, $p<.01$., CAS subscale, $p$ $<.05$, and CCB subscale, $p<.05$ ). In further analysis by correlations with diversity training in all categories, no significant findings were evident. However, "continuing education" and "online computer-assisted education" diversity training were shown to be significantly associated with cultural awareness and sensitivity $(p<.05)$.

These findings were supported in the literature, noting that conceptual meaning of continuing education can be construed as professional seminars/conferences (Starr \& Wallace, 2009). In the present study and that of Starr and Wallace (2009), researchers found a significant relationship with online computer-assisted education and cultural 
awareness and sensitivity. However, other types of diversity training namely, separate college course for credit, content covered in a course, professional conference/seminar, or employer-sponsored programs did not relate to any of the three measures of cultural competency.

Significant relationships were found between program level and the measure of cultural awareness and sensitivity (CAS), $p<.05$. The higher program standing (GPA 3.5-4.0) group was significantly associated with higher students' overall cultural competency (total CCA), $p<.01$ and, with culturally competent behaviors (CCB subscale) $p<.01$. Similarly, significant relationships between the numbers of types of diversity training attended were found with all three measures of cultural competency (total CCA, $p<.01$, the CAS, $p<.01$, and the CCB, $p<.05$ ). Regression analyses completed the evaluation of relationships between the number of previous diversity training types (maximum of 6) and the three measures of cultural competency as the dependent variables. For the three regression models, one for each dependent variable, the first block added age, gender, ethnicity/race, and language spoken at home, was found to be not significant. However, the second block added program level (graduate) and program standing (GPA 3.50-4.00) for all three equations. The present study had shown evidence that program level, program standing, and the number of previous diversity training types attended, are significant predictors of overall cultural competence, cultural awareness and sensitivity, as well as culturally competent behaviors.

\section{Conclusion}

The research hypotheses in this study were largely supported theoretically by the literature and statistically by applying correlation, one-way ANOVA, and multiple 
regression techniques. Briefly, multiple regression analysis, after controlling for all predictors, found program level, program standing, and diversity training explained a significant amount of variance in cultural competency $\left(p=.027 ; R^{2}=.18\right)$. Continuing education is crucial in achieving students' cultural competency. Previous diversity training, graduate education, and higher grade-point average were correlated with higher cultural competency levels. However, increased diversity encounters were not associated with higher cultural competency levels.

Findings from this study support a future initiative for continuing education on diversity training. Continuing education may use online computer-assisted education, culture-focused courses, and systematically planned seminars or conferences. . Students' program level and academic standing have conceptual aspects that need further investigation to explain significant differences between undergraduate students' and graduate students' educational experiences. There may be other forces involved, such as higher comprehension of material associated with higher program standing (GPAs). The findings of this study are potentially valuable in the planning, implementation, or revision of undergraduate and graduate level curricula, as well as continuing-education topics and future requirements associated with increased nursing students cultural competency levels. This study is timely and important for university nursing programs with undergraduate and graduate level students to maintain AACN and/or NLN accreditation. It may help universities adequately prepare nursing students' to care for the changing and diverse patient populations they are likely to encounter in practice, from novice to expert, as a student, clinician, educator, or researcher. 


\section{Implications}

Nursing policy. Research findings from this study reinforce the statewide policy and requirement of licensed nurses and other healthcare workers to participate in continuing education as a condition for continued licensure. Another professional policy and initiative is to increase the minimum educational level of nurses to baccalaureate degree (Benner, Sutphen, Leonard, \& Day, 2010). The findings from this study support the initiative and the benefits of higher educational levels related to cultural competency outcomes. Therefore, it behooves all stakeholder, patients, communities, and administrators to include cultural-diversity training in professional nurses' continuing education requirements, implemented by professional nursing agencies and enacted by state legislatures. This research suggests that the requirement of diversity training is valuable and might result in increased awareness and cultural competency of licensed nursing graduates.

Nursing practice. Research findings from this study imply nursing graduates entering clinical practice should continue to receive continuing education with choices of diversity-training topics offered by employers or outside professional educational companies. The study findings suggest a strong relationship between cultural competency levels and continuing education that should be included in all healthcare settings and work places as part of orientation, ongoing conferences, seminars, or computer-assisted education. Continued assessment of graduates, once licensed and practicing as clinicians, would be useful, to learn if cultural awareness/knowledge and sensitivity, performance of culturally competent behavior, and overall cultural competency, transform into practice and remain ongoing. Cultural competency is a dynamic process and not an endpoint. 
Findings from this study are valuable to various stakeholders: nursing students, faculty, program directors, administrators, online and continuing-education companies, and the recipients of care from nursing students, such as patients, families, and communities. An annual skill assessment using the CCA tool in healthcare environments and alternate patient-care settings merits consideration.

Nursing education. This study strongly supports higher education levels and higher academic standing, which are associated with higher cultural competency levels. Clearly, cultural competency levels are not associated with the specific nuances of specialty tracks, but with overall higher levels of education (MSN) and higher grades (GPAs). Experiential-learning theory support and guide nursing programs' local community health courses and international immersions evaluated by researchers. Although diverse client care is believed to be a crucial practice in students' process toward cultural competence, this study supports increased educational modalities that build knowledge and weigh heavily on students' cultural competency outcomes. It is proposed that engaging students in active learning pedagogy to achieve affective, cognitive, and culturally competent behavioral learning outcomes while in school would result in students' attainment of higher cultural competency levels, enabling them to advance into effective clinical practice upon graduation and beyond. Matching students (mentees) of lower program standing with students (mentor) of higher academic standing will facilitate students' learning process and development of overall cultural competency levels. 


\section{Limitations}

Experts agree that convenience samples and self-reported data limit generalizations and cannot control bias (Polit, 2010; Waltz, Strickland, \& Lenz, 2010). The sample was derived from just one institution, with a predominantly Hispanic student population, and limited to nursing students enrolled in one college. Caution should be exercised when making generalizations from this study. A larger response rate would have increased the significance of this research study. Of note, the respondents reflected difficulty with completion of fill-in responses that limited the use of two of the encountered questions. The recommendations below suggest ways to improve development of future studies.

\section{Recommendations for Future Research}

More research is needed to evaluate the unexpected lack of cultural competency differences between program level and program track groups on measures of overall cultural competency, cultural awareness and sensitivity, and culturally competent behavior. Future research with the CCA tool should include an item/question about respondents' previous completion of community-health courses and participation in international travel, as these items were useful in other evaluations of cultural competency and could easily be added to demographic questions (Bernal \& Froman, 1993; Bond, et al. 2001; Brathwaite, 2005; Hagman, 2006; Jeffreys \& Dogan, 2010; Jones et al., 2004). Future study should include student samples from more than one institution, perhaps across the United States and the globe. Further inquiry should include qualitative assessment to learn of the lived experiences of the uncustomary FEP BSN/MSN student group, as well as experiences with homeless/ 
housing insecure clients. A recommendation to increase the response rate of future research with the electronic CCA includes securing funds for use of a downloadable coupon as incentive or to increase students' motivation to complete surveys.

Research should include multidisciplinary healthcare student samples composed of: medical, pharmacy, physical/occupational, and speech-therapy student groups. These groups could be enrolled in this southeast, research intensive, minority/Hispanic-serving public university or other institutions of higher learning, including public or private colleges, as part of collaborative research projects.

This study's findings support the implementation of a culture course for undergraduates. To further support a stand-alone culture course for undergraduate nursing students, future research should use an experimental design with an elective culture course as the intervention. Evaluation could follow with comparative research between those who completed the elective culture course with those who had not.

Future research could be guided by the three-dimensional CCA model and data could be collected with the CCA scale. Consultation with the tool's creator should be done to discuss a potential new format for data collection of the number of previous diversity encounters and the like. The sample in this study seemed to have difficulty completing the items that were not part of the psychometrically tested scale. 


\section{LIST OF REFERENCES}

Abrums, M., \& Leppa, C. (2001). Beyond cultural competence: Teaching about race, gender, class, and sexual orientation. Journal of Nursing Education, 40, 270-275.

Alpers, R., \& Zoucha, R. (1996). Comparison of cultural competence and cultural confidence of senior nursing students in a private southern university. Journal of Cultural Diversity, 3, 9-15.

American Association of Colleges of Nursing. (2008a). The essentials of baccalaureate education for professional nursing practice. Washington, DC: Author.

American Association of Colleges of Nursing. (2008b). Tool kit of resources for cultural competent education for baccalaureate nurses. Retrieved from http://www.aacn .nche.edu/education-resources/toolkit.pdf

American Association of Colleges of Nursing. (2011). Fact sheet. Retrieved from http:// www.aacn.nche.edu/media-relations/fact-sheets/nursing-fact-sheet

American Nurses Association. (1986). Cultural diversity in the nursing curriculum: A guide for implementation. Kansas City, MO: American Nurses Association.

American Nurses Association. (1991). Position statement on cultural diversity in nursing practice. Retrieved from http://www.nursingworld.org/MainMenuCategories /Policy-Advocacy/Positions-and-Resolutions/ANAPositionStatements/Position -Statements-Alphabetically/prtetcldv14444.html

Amerson, R. (2010). The impact of service-learning on cultural competence. Nursing Education Perspectives, 31, 18-22. doi:10.1043/1536-5026-31.1.18

Anderson, K. L. (2004). Teaching cultural competence using an exemplar from literary journalism. Journal of Nursing Education, 43, 253-259.

Anderson, L.W. \& Krathwohl, D. (Eds.) (2001). A taxonomy for learning, teaching and assessing: A revision of Bloom's taxonomy of educational objectives. Longman: NY.

Andrews, M. M., \& Boyle, J. S. (2008). Transcultural concepts in nursing care (5th ed.). Philadelphia, PA: Wolters Kluwer Health.

Assemi, M., Cullander, C., \& Hudmon, K. S. (2004). Implementation and evaluation of cultural competency training for pharmacy students. Annals of Pharmacotherapy, 38, 781-786. doi:10.1345/aph.1D402 
Baldonado, A., Beymer, P., Barnes, K., Starsiak, D. Nemivant, E., \& Anonas-Ternate, A. (1998). Transcultural nursing practice described by registered nurses and baccalaureate nursing students. Journal of Transcultural Nursing, 9, 234-241. doi:10.1177/104365969800900204

Bandura, A. (1977). Self-efficacy: Toward a unifying theory of behavioral change. Psychological Review, 84, 191-215. doi:10.1037/0033-295X.84.2.191

Bartol, G. M., \& Richardson, L. (1998). Using literature to create cultural competence. Image, 30(1), 75-79. doi:10.1111/j.1547-5069.1998.tb01240.x

Barton, J. A., \& Brown, N. J. (1992). Evaluation study of a transcultural discovery learning model. Public Health Nursing, 9, 234-241. doi:10.1111/j.1525-1446 .1992.tb00107.x

Bassi, S. (2011). Undergraduate nursing students' perceptions of service-learning through a school-based community project. Nursing Education Perspectives, 32(3), 162167.

Bednarz. H., Schim, S., \& Doorenbos, A. (2010). Cultural diversity in nursing education. Journal of Nursing Education, 49, 253-260. doi:10.3928/01484834-20100115-02

Benkert, R., Tanner, C., Guthrie, B., Oakley, D., \& Pohl, J. M. (2005). Cultural competence of nurse practitioner students: A consortium's experience. Journal of Nursing Education, 44, 225-233.

Benkert, R., Templin, T., Schim, S., Doorenbos, A., \& Bell, S. (2011). Testing a multigroup model of culturally competent behaviors among underrepresented nurse practitioners. Research in Health \& Nursing, 34, 327-341. doi:10.1002/nur.20441

Benner, P. (1982). From novice to expert. American Journal of Nursing, 82, 402-407. doi: $10.2307 / 3462928$

Benner, P. (2001). From novice to expert: Excellence and power in clinical nursing practice. Upper Saddle River, NJ: Prentice Hall Health.

Benner, P., Sutphen, M., Leonard, V., \& Day, L. (2010). Educating nurses: A call for radical transformation. San Francisco, CA: Jossey Bass.

Berlin, E. A., \& Fowkes, W. C. (1983). A teaching framework for cross-cultural health care: Application in family practice. Western Journal of Medicine, 139, 934-938.

Bernal, H., \& Froman, R. (1987). The confidence of community health nurses caring for ethnically diverse populations. Journal of Nursing Scholarship, 19, 201-204. doi: 10.1111/j.1547-5069.1987.tb00008.x 
Bernal, H., \& Froman, R. (1993). Influences on the cultural self-efficacy of community health nurses. Journal of Transcultural Nursing, 4, 24-31. doi:10.1177 $/ 104365969300400205$

Betancourt, J. R. (2006, October 17). Improving quality and achieving equity: The role of cultural competence in reducing racial and ethnic disparities in health care. The Commonwealth Fund, 37. Retrieved from http://www.commonwealthfund.org /Publications/Fund-Reports/2006/Oct/Improving-Quality-and-Achieving-Equity --The-Role-of-Cultural-Competence-in-Reducing-Racial-and-Ethni.aspx

Betancourt, J. R. (2007). Commentary on: Current approaches to integrating elements of cultural competence in nursing education. Journal of Transcultural Nursing, 18, 25S-27S. doi:10.1177/1043659606296120

Bloom, B. S. (1956). Taxonomy of educational objectives: The classification of educational goals. New York: Longmans, Green.

Bond, M. L. (2004). Testimony to the Sullivan Commission: Diversity in the healthcare workforce. Washington, DC: U.S. Department of Health and Human Services.

Bond, M., \& Jones, M. (1994). Short-term cultural immersion in Mexico. Nursing \& Health Care, 15, 248-253.

Bond, M. L., Kardong-Edgren, \& Jones, M. E. (2001). Assessment of professional nursing students' knowledge and attitudes about patients of diverse cultures. Journal of Professional Nursing, 17, 305-312. doi:10.1053/jpnu.2001.28426

Brathwaite, A. (2005). Evaluation of a cultural competence course. Journal of Transcultural Nursing, 16, 361-369. doi:10.1177/1043659605278941

Brathwaite, A. (2006). Influence of nurse characteristics on the acquisition of cultural competence. International Journal of Nursing Education Scholarship, 3(1). doi: 10.2202/1548-923X.1173

Brennan, A. M., \& Cotter, V. T. (2008). Student perceptions of cultural competence content in the curriculum Journal of Professional Nursing, 24, 155-160. doi:10 $.1013 /$ j.profnurs.2008.01.003

Brennan, S. J., \& Schulze, M. W. (2004). Cultural immersion through ethnography: The lived experience and group process. Journal of Nursing Education, 43, 286-288.

Buerhaus, P. (2008). Current and future state of U.S. nursing workforce. Journal of the American Medical Association, 300, 2422-2424. doi:10.1001/jama.2008.729 
Caffrey, R. A., Neander, W., Markle, D., \& Stewart, B. (2005). Improving cultural competence of nursing students: Results of integrating cultural content in the curriculum and an international immersion experience. Journal of Nursing Education, 44, 234-240.

Callister, L., \& Cox, A. (2006). Opening our hearts and minds: The meaning of international clinical nursing electives in the personal and professional lives of nurses. Nursing and Health Sciences, 8, 95-102. doi:10.1111/j.14422018.2006 $.00259 . \mathrm{x}$

Campbell-Heider, N., Rejman, K. P., Austin-Ketch, T., Sackett, K., Feeley, T. H., \& Wilk, N. C. (2006). Measuring cultural competence in a family nurse practitioner curriculum. Journal of Multicultural Nursing \& Health, 12(3), 24-34.

Campinha-Bacote, J. (1999). A model and instrument for addressing cultural competence in health care. Journal of Nursing Education, 38, 204-207.

Campinha-Bacote, J. (2002). The process of cultural competence in the delivery of healthcare services: A model of care. Journal of Transcultural Nursing, 13, 181184. doi: $10.1177 / 10459602013003003$

Campinha-Bacote, J. (2007). The process of cultural competence in the delivery of healthcare services: The journey continues (5th ed.). Cincinnati, OH: Transcultural C.A.R.E. Associates.

Carpio, B. A., \& Majumdar, B. (1993). Experiential learning: An approach to transcultural education for nursing. Journal of Transcultural Nursing, 4, 4-11. doi:10.1177/104365969300400202

Clark, L., Zuk, J., \& Baramee, J. (2000). A literary approach to teaching cultural competence. Journal of Transcultural Nursing, 11, 199-203. doi:10.1177 /104365960001100306

Coffman, M., Shellman, J., \& Bernal, H. (2004). An integrative review of American nurses' perceived cultural self-efficacy. Journal of Nursing Scholarship, 36, 180185. doi:10.1111/j.1547-5069.2004.04032.x

Cohen, J., Cohen, P., West, S., \& Aiken, L. (2003). Applied multiple regression/ correlation analysis for the behavioral sciences. (3rd ed.). Mahwah, NJ: Lawrence Erlbaum.

Cooper, L. A., Roter, D. L., Bone, L. R., Larson, S. M., Miller, E. R., Barr, M. S., ,.. Levine, D. M. (2009). A randomized controlled trial of interventions to enhance patient physician partnership, patient adherence and high blood pressure control among ethnic minorities and poor persons: Study protocol NCT00123045. Implementation Science, 4(7), 1-16. doi:10.1186/1748-5908-4-7 
Cortis, J. D. (2000). Caring as experienced by minority ethnic patients. International Nursing Review, 47, 53-62. doi:10.1046/j.1466-7657.2000.00006.x

Cross, T., Bazron, B., Dennis, K., \& Isaacs, M. (1989). Towards a culturally competent system of care (Vol. 1). Washington, DC: Georgetown University Child Development Center.

Cuellar, N. G., Brennan, A. M., Vito, K., \& Siantz, M. L. (2008). Cultural competence in the undergraduate nursing curriculum. Journal of Professional Nursing, 24, 143149. doi:10.

1016/j.profnurs.2008.01.004

Doorenbos, A., Lindhorst, T., Schim., S., Schaik, E., Demiris, G., Wechkin, H., \& Curtis, J. (2010). Development of a web-based educational intervention to improve crosscultural communication among hospice providers. Journal of Social Work in Endof-Life \& Palliative Care, 6, 236-255. doi:10.1080/15524256.2010.529022

Doorenbos, A., \& Schim, S. M. (2004). Cultural competence in hospice. American Journal of Hospice \& Palliative Care, 21, 28-32. doi:10.1177/ 104990910402100108

Doorenbos, A., Schim, S. M., Benkert, R., \& Borse, N. (2005). Psychometric evaluation of the cultural competence assessment instrument among healthcare providers. Nursing Research, 54, 324-331. doi:10.1097/00006199-200509000-00006

Douglas, M. K., \& Pacquiao, D. F. (2010). Core curriculum in transcultural nursing and health care. Journal of Transcultural Nursing, 21, 373S-405S. doi:10.1177/ 1043659610374321

Douglas, M. K., Pierce, J. U., Rosenkoetter, M., Pacquiao, D., Callister, L. C., HattarPollara, M., Lauderdale, J., ... Purnell, L. (2011). Standards of practice for culturally competent nursing care: 2011 update. Journal of Transcultural Nursing, 22, 317-333. doi:10.1177/1043659611412965

Doutrich, D., \& Storey, M. (2004). Education and practice: Dynamic partners for improving cultural competence in public health. Family \& Community Health, 27, 298-307. doi:10.1097/00003727-200410000-00006

Duffy, M. E., Farmer, S., Ravert, P., \& Huittinen, L. (2003). Institutional issues in the implementation of an international student exchange program. Journal of Nursing Education, 42, 399-405.

Duke, J., Connor, M., \& McEldowney, R. (2009). Becoming a culturally competent health practitioner in the delivery of culturally safe care: A program oriented approach. Journal of Cultural Diversity, 16, 40-49. 
Ehrenfeld, M., Shmueli, M., \& Henig, M. (1998). Absorption of nursing students: New immigrants in the general academic nursing programme in Israel. Journal of Advanced Nursing, 27, 171-178. doi:10.1046/j.1365-2648.1998.00495.x

Eliason, M. J., \& Raheim, S. (2000). Experiences and comfort with culturally diverse groups in undergraduate pre-nursing students. Journal of Nursing Education, 39, $161-165$.

Ervin, N. E., Bickes, J. T., \& Schim, S. M. (2006). Environments of care: A curriculum model for preparing a new generation of nurses. Journal of Nursing Education, 45, $75-80$.

Fadiman, A. (1997). The spirit catches you and you fall down: A Hmong child, her American doctors, and the collision of two cultures. New York, NY: Farrar, Straus, \& Giroux.

Fahrenwald, N. L., Boysen, R., Fischer, C., \& Maurer, R. (2001). Developing cultural competence in the baccalaureate nursing student: A population-based project with the Hutterites. Journal of Transcultural Nursing, 12, 48-55. doi:10.1177/ 104365960101200107

Family Educational Rights and Privacy Act (FERPA) (20 U.S.C. § 1232g; CFR Part 99 (nd). U.S. Department of Education, Washington, D.C. Retrieved from http:// www.ed.gov/policy/gen/guid/fpco/ferpa/index.html

Felder, E. (1990). Baccalaureate and associate degree student nurses' cultural knowledge of and attitudes toward Black Americans. Journal of Nursing Education, 29, 276282.

Fitzgerald, E. M., Cronin, S. N., \& Campinha-Bacote, J. (2007). Psychometric testing of the Inventory for Assessing the Process of Cultural Competence among healthcare professionals-Student version (IAPCC-SV). Journal of Theory Construction \& Testing, 13, 64-68.

Fortier, J. P., \& Bishop, D. (2003). Setting the agenda for research on cultural competence in healthcare. Retrieved from http://www.ahrq.gov/research /findings/factsheets/literacy/cultural/cultural.pdf

Galanti, G. (2008). Caring for patients from different cultures (4th ed.). Philadelphia: University of Pennsylvania Press.

Gaskin, J. G., \& Hoffman, C (2000). Racial and ethnic differences in preventable hospitalizations across 10 states. Medical Care Research and Review, 57, S85S107. doi:10.1177/107755800773743619 
Gebru, K., Ahsberg, E., \& Willman, A. (2007). Nursing and medical documentation. Journal of Clinical Nursing, 16, 2056-2065. doi:10.1111/j.1365-2702.2006 .01645

Giger, J,. \& Davidhizar, R. (2008). Transcultural nursing: Assessment and intervention. (5th ed.). St. Louis, MO: Mosby Elsevier.

Goode, T., Dunne, C., \& Bronheim, S. (2006). The evidence base for cultural and linguistic competency in health care. Retrieved from http://www .commonwealthfund.org/usr_doc/Goode_evidencebasecultlinguisticcomp_962 .pdf

Graham, I., \& Richardson, E. (2008). Experiential gaming to facilitate cultural awareness: Its implication for developing emotional caring in nursing. Learning in Health \& Social Care, 7, 37-45. doi:10.1111/j.1473-6861.2008.00168.x

Green, S., Comer, L., Elliott, L., \& Neubrander, J. (2011). Exploring the value of an international service-learning experience in Honduras. Nursing Education Perspectives, 32, 302-307. doi:10.5480/1536-5026-32.5.302

Grossman, D., Massey, P., Blais, K., Geiger, E., Lowe, J., Pereira, O., ... Tally-Ross, N. (1998). Cultural diversity in Florida nursing programs: A survey of deans and directors. Journal of Nursing Education, 37, 22-26.

Guttman, M. (2004). Increasing the linguistic competence of the nurse with limited English proficiency. Journal of Continuing Education in Nursing, 35, 264-269.

Hagman, L. (2006). Cultural self-efficacy of licensed registered nurses in New Mexico. Journal of Cultural Diversity, 13, 105-112.

Halloran, L. (2009). Teaching transcultural nursing through literature. Journal of Nursing Education, 48, 523-528.

Hertel, J., \& Millis, B. (2002). Using simulation to promote learning in higher education: An introduction. Sterling, VA: Stylus.

Heuer, L., Bengiamin, M., \& Downey, V. W. (2001). The impact of an international cultural experience on previously held stereotypes of American student nurses. Multicultural Education, 9(1), 26-29.

Hoeffer, M., Rytina, N., \& Baker, B. C. (2010). Estimates of the unauthorized immigrant population residing in the United States: January 2010. Retrieved from http:// www.dhs.gov/xlibrary/assets/statistics/publications/ois_ill_pe_2010.pdf

Hughes, K. H., \& Hood, L. J. (2007). Teaching methods and an outcome tool for measuring cultural sensitivity in undergraduate nursing students. Journal of Transcultural Nursing, 18, 57-62. doi:10.1177/1043659606294196 
Hunter, J. L. (2008). Applying constructivism to nursing education in cultural competence: A course that bears repeating. Journal of Transcultural Nursing, 19, 354-362. doi:10.1177/1043659608322421

Hunter, J. L. , \& Krantz, S. (2010). Constructivism in cultural competence education. Journal of Nursing Education, 49, 207-214.

Institute of Medicine. (2003). Health professions education: A bridge to quality. Washington, DC: National Academies Press.

Jeffreys, M. R. (2000). Development and psychometric evaluation of the transcultural self-efficacy tool: A synthesis of findings. Journal of Transcultural Nursing, 11, 127-136. doi:10.1177/104365960001100207

Jeffreys, M. R. (2010). Teaching cultural competence in nursing and health care (2nd ed.). New York, NY: Springer.

Jeffreys, M. R., \& Dogan, E. (2012). Evaluating the influence of cultural competence education on students' transcultural self-efficacy perceptions. Journal of Transcultural Nursing, 23, 188-197. doi:10.1177/1043659611423836

Jenakovich, M., Puebla Fortier, J., Crain, C., Anderson, P., Madden, M., \& Kong, J. (2001). Cultural competence works: Using cultural competence to improve the quality of health care for diverse populations and add value to managed care arrangements. Retrieved from http://minority-health.pitt.edu/278/1/cultural -competence_works-(assessment_tool).pdf

Joint Commission. (2010). Cultural and linguistic care in area hospitals: An exploration of the tools, resources, services and practices used by hospitals in Florida's Martin, Palm Beach and St. Lucie counties. Retrieved from http://www.jointcommission .org/assets/1/18/FINAL_REPORT_MARCH_2010.pdf

Jones, M. E., Cason, C. L., \& Bond, M. L. (2004). Cultural attitudes, knowledge, and skills of a health workforce. Journal of Transcultural Nursing, 15, 283-290. doi: $10.1177 / 1043659604268966$

Junious, D., Malecha, A., Tart, K., \& Young, A. (2010). Stress and perceived faculty support among foreign-born baccalaureate nursing students. Journal of Nursing Education, 49, 261-270.

Kardong-Edgren, S. (2007). Cultural competence of baccalaureate nursing faculty. Journal of Nursing Education, 46, 360-366.

Kardong-Edgren, S., \& Campinha-Bacote, J. (2008). Cultural competency of graduating U.S. bachelor of science nursing students. Contemporary Nurse, 28, 37-44. doi: 10.5172/conu.673.28.1-2.37 
Kardong-Edgren, S., Cason, C. L., Brennan, A. M., Reifsnider, E., Hummel, F., Mancini, M., \& Griffin, C. (2010). Cultural competency of graduating BSN nursing students. Nursing Education Perspectives, 31, 279-285.

Kaufman, K. A. (2010). Findings from the annual survey of schools of nursing academic year 2008-2009: Students are increasingly diverse but lack of educational capacity still stymies enrollment growth. Nursing Education Perspectives, 31, 196-197.

Kavanagh, K., Absalom, K., Beil, W., \& Schliessmann, L. (1999). Connecting and becoming culturally competent: A Lakota example. Advances in Nursing Science, 21(3), 9-31. Retrieved from Wolters Kluwer database.

Kennedy, H. P., Fisher, L., Fontaine, D., \& Martin-Holland, J. (2008). Evaluating diversity in nursing education: A mixed method study. Journal of Transcultural Nursing, 19, 363-370. doi:10.1177/1043659608322500

Kleinman, A. (1978). Concepts and a model for the comparison of medical systems as cultural systems. Social Science and Medicine, 12(2-B), 85-93. doi:10.1016 /S0277-9536(78)80014-8

Kolb, D. (1984). Experiential learning theory: Experience as the source of learning and development. Upper Saddle River, NJ: Prentice Hall.

Kollar, S., \& Ailinger, R. (2002). International clinical experiences: Long-term impact on students. Nurse Educator, 27, 28-31.

Krainovich-Miller, B., Yost, J. M., Norman, R. G., Auerhan, C., Dobal, M., Rosedale, M., Lowry, M., \& Moffa, C. (2008). Measuring cultural awareness of nursing students: A first step toward cultural competency. Journal of Transcultural Nursing, 19, 250-258. doi:10.1177/1043659608317451

Kulwicki, A., \& Boloink, B. (1996). Assessment of level of comfort in providing multicultural nursing care by baccalaureate nursing students. Journal of Cultural Diversity, 3, 40-45.

Larson, K., Ott, M., \& Miles, J. (2010). International cultural immersion: En vivo reflections in cultural competence. Journal of Cultural Diversity, 17, 44-50. Retrieved from http://www.readperiodicals.com/201007/2054111121.html\#b

Leininger, M. (1988). Leininger's theory of nursing: Cultural care diversity and universality. Nursing Science Quarterly, 1, 152-156. doi:10.1177 /089431848800100408

Leininger, M. (1995). Teaching transcultural nursing in undergraduate and graduate programs. Journal of Transcultural Nursing, 6, 10-26. doi:10.1177 /104365969500600203 
Leininger, M., \& McFarland, M. (2006). Culture care diversity and universality: A worldwide nursing theory (2nd ed.). Sudbury, MA: Jones \& Bartlett.

Leiper, J., Van Horn, E., Hu, J., \& Upadhyaya, R. (2008). Promoting cultural awareness and knowledge among faculty and doctoral students. Nursing Education Perspectives, 29, 161-164. Retrieved from http://libres.uncg.edu/ir/uncg/f /J_Hu_Promoting_2008(MULTI\%20UNCG\%20AUTHORS).pdf

Leonard, T. (2006). Exploring diversity in nursing education: Research findings. Journal of Cultural Diversity, 13, 87-96.

Levin, S., Like, R., \& Gottlieb, J. (2000). ETHNIC: A framework for culturally competent clinical practice. Patient Care, 34(9), 188-189.

Lim, J., Downie, J., \& Nathan, P. (2004). Nursing students' self-efficacy in providing transcultural care. Nurse Education Today, 24, 428-434. doi:10.1016/j.nedt.2004 .04 .007

Lockhart, J. S., \& Resick, L. K. (1997). Teaching cultural competence: The value of experiential learning and community resources. Nurse Educator, 22, 27-31. doi: 10.1097/00006223-199705000-00014

Memmott, R. J., Coverston, C. R., Heise, B. A., Williams, M., Maughan, E. D., Kohl, J., \& Palmer, S. (2010). Practical considerations in establishing sustainable international nursing experiences. Nursing Education Perspectives, 31, 298-302.

Mixer, S. J. (2008). Use of the cultural care theory and ethnonursing method to discover how nursing faculty teach culture care. Contemporary Nurse, 28, 23-36. doi:10 $.5172 /$ conu.673.28.1-2.23

Mixer, S. J. (2011). Use of the culture care theory to discover nursing faculty care expressions, patterns, and practices related to teaching culture care. The Online Journal of Cultural Competence in Nursing and Healthcare, 1(1), 3-14. Retrieved from http://www.ojccnh.org/1/1/3-14.pdf

Munoz, C. C., \& Luckmann, J. (2005). Transcultural communication in nursing (2nd ed.). Clifton Park, NY: Delmar Learning.

Munro, B. H. (2005). Statistical methods for health care research (5th ed.) Philadelphia, PA: Lippincott Williams \& Wilkins.

National League for Nursing. (2008). Annual survey of schools of nursing academic year 2005-2006: Executive summary. Retrieved from http://www.nln.org/research /slides/exec_summary.htm. 
Paez, K. A., Allen, J. K., Carson, K. A., \& Cooper, L. A. (2008). Provider and clinic cultural competence in a primary care setting. Social Science \& Medicine, 66, 1204-1216. doi:10.1016/j.socscimed.2007.11.027

Papadopoulos, I., Tilki, M., \& Ayling, S. (2008). Cultural competence in action for CAMHS: Development of a cultural competence assessment tool and training programme. Contemporary Nurse, 28, 129-140.

Papps, E., \& Ramsden, I. (1996). Cultural safety in nursing: The New Zealand experience. International Journal of Quality in Health Care, 8, 491-497. doi:10.1093/intqhe /8.5.491

Polit, D. E. (2010). Statistics and data analysis for nursing research (2nd ed.). Upper Saddle River, NJ: Pearson Education.

Purnell, L. D. (2002). The Purnell model for cultural competence. Journal of Transcultural Nursing, 13, 193-196. doi:10.1177/10459602013003006

Purnell, L. (2005). The Purnell model for cultural competence. Journal of Multicultural Nursing \& Health, 11(2), 7-15.

Ramsden, I. \& Spoonley, P. (1994). The cultural safety debate in nursing education in Aotearoa. New Zealand Annual Review of Education, 3, 161-174.

Reeves, J., \& Fogg, C. (2006). Perceptions of graduating nursing students regarding life experiences that promote culturally competent care. Journal of Transcultural Nursing, 17, 171-178. doi:10.1177/1043659605285410

Reneau, M. (2013). Teaching nurses sight unseen: Comparing the cultural competency of online and on-campus BSN faculty. Journal of Transcultural Nursing, 24, 78-85. doi:10.1177/1043659612462405

Rew, L. (1996). Affirming cultural diversity: A pathways model for nursing faculty. Journal of Nursing Education, 35, 310-314.

Riley, D. M. (2010). Cultural competence of RN to BSN students (Doctoral dissertation). University of Nevada, Las Vegas. Retrieved from http://digitalscholarship.unlv .edu/cgi/viewcontent.cgi?article $=1369 \&$ amp; context $=$ thesesdissertations

Riner, M. E., \& Becklenberg, A. (2001). Partnering with a sister city organization for an international service-learning experience. Journal of Transcultural Nursing, 12, 234-240. doi:10.1177/104365960101200308

Rooda, L., \& Gay, G. (1993). Staff development for culturally sensitive nursing care. Journal of Nursing Staff Development, 9(6), 262-265. 
Rosenberg, M. (n.d.). Names of generations: Generational names in the United States. Retrieved from http://geography.about.com/od/populationgeography/qt /generations.html

Rosenstoch, I. (1974). Historical origin of Health Belief model. Health Education Monograph, 2, 334.

Ryan, M., Carlton, K., \& Ali, N. (2000). Transcultural nursing concepts and experiences in nursing curricula. Journal of Transcultural Nursing, 11, 300-307. doi:10.1177 $/ 104365960001100408$

Ryan, M., \& Twibell, R. (2002). Outcomes of a transcultural nursing immersion experience: Confirmation of a dimensional matrix. Journal of Transcultural Nursing, 13, 30-39. doi:10.1177/104365960201300106

Sanner, S., Baldwin, D., Cannella, K., Charles, J., \& Parker, L. (2010). The impact of cultural forum on students' openness to diversity. Journal of Cultural Diversity, 17, 56-61.

Sargent, S., Sedlak, C., \& Marstolf, D. (2005). Cultural competence among nursing students and faculty. Nurse Education Today, 25, 214-225. doi:10.1016/j.nedt .2004.12.005

Schim, S. M., \& Doorenbos, A. Z. (2010). A three-dimensional model of cultural congruence: Framework for intervention. Journal of Social Work in End-of-Life \& Palliative Care, 6, 256-270. doi:10.1080/15524256.2010.529023

Schim, S. M., Doorenbos, A. Z., Benkert, R., \& Miller, J. (2007). Culturally congruent care: Putting the puzzle together. Journal of Transcultural Nursing, 18, 103-110. doi:10.1177/1043659606298613

Schim, S. M., Doorenbos, A. Z., \& Borse, N. (2005). Cultural competence among Ontario and Michigan healthcare providers. Journal of Nursing Scholarship, 37, 354-360. doi:10.1111/j.1547-5069.2005.00061.x

Schim, S. M., Doorenbos, A. Z., \& Borse, N. (2006a). Cultural competence among hospice workers. Journal of Hospice \& Palliative Nursing, 8, 302-307. doi:10 .1097/00129191-200609000-00016

Schim, S. M., Doorenbos, A. Z., \& Borse, N. (2006b). Enhancing cultural competence among hospice staff. American Journal of Hospice \& Palliative Medicine, 23, 404-411. doi10.1177/1049909106292246

Schim, S. M., Doorenbos, A. Z., Miller, J., \& Benkert, R. (2003). Development of a cultural competence assessment instrument. Journal of Nursing Measurement, 11, 29-40. doi:10.1891/jnum.11.1.29.52062 
Shearer, R., \& Davidhizar, R. (2003). Using role-play to develop cultural competence. Journal of Nursing Education, 42, 273-276.

Smedley, B. D., Stith, A. Y., \& Nelson, A. R. (Eds.). (2002). Unequal treatment: Confronting racial and ethnic disparities in healthcare. Retrieved from http:// www.nap.edu/catalog/10260.html

Smith, L. S. (1998). Evaluation of an educational intervention to increase cultural competence among registered nurses. Journal of Cultural Diversity, 8, 50-63.

Soto-Greene, M. L., Sanchez, J., Salas-Lopez, D., \& Like, R. (2004). Antecedents to effective treatment of hypertension in Hispanic populations. Clinical Cornerstone, 6(3), 30-38. doi:10.1016/S1098-3597(04)80062-0

Spector, R. E. (2009). Cultural diversity in health and illness (7th ed.). Upper Saddle River, NJ: Pearson Prentice Hall.

Starr, S., \& Wallace, D. (2009). Self-reported cultural competence of public health nurses in a southeastern U.S. public health department. Public Health Nursing, 26, 48-57. doi:10.1111/j.1525-1446.2008.00753.x

St. Clair, A., \& McKendry, L. (1999). Preparing culturally competent practitioners. Journal of Nursing Education, 38, 228-234.

Tuck, I., Moon, M. W., \& Allocca, P. N. (2010). An integrative approach to cultural competence education for advanced practice nurses. Journal of Transcultural Nursing, 21, 402-409. doi:10.1177/1043659609360716

Tulman, L., \& Watts, R. J. (2008). Development and testing of the blueprint for integration of cultural competence in the curriculum questionnaire. Journal of Professional Nursing, 24, 161-166. doi:10.1016/j.profnurs.2008.01.006

U.S. Census Bureau. (2010). Census 2010. Retrieved from http://www2.census.gov/census_2010/03Demographic_Profile

U.S. Department of Health and Human Services. (2010). The registered nurse population: Findings from the 2008 national sample survey of registered nurses. Retrieved from http://bhpr.hrsa/gov/healthworkforce/allreports.html

U.S. Department of Health and Human Services, Office of Minority Health. (2001). National standards on culturally and linguistically appropriate services (CLAS). Retrieved from http://minorityhealth.hhs.gov/assets/pdf/checked/finalreport.pdf

Walsh, L. V., \& DeJoseph, J. (2003). I saw it in a different light: International learning experiences in baccalaureate nursing education. Journal of Nursing Education, 42, 266-272. 
Waltz, C. F., Strickland, O. L., \& Lenz, E. R. (2010). Measurement in nursing and health research (4th ed.). New York, NY: Springer.

Wells, M. I. (2000). Beyond cultural competence: A model for individual and institutional cultural development. Journal of Community Health Nursing, 17, 189-199. doi:10.1207/S15327655JCHN1704_1

Wilson, A. H., Sanner, S., \& McAllister, L. E. (2010). A longitudinal study of cultural competence among health science faculty. Journal of Cultural Diversity, 17, 6872.

Yoder, M. (1996). Instructional responses to ethnically diverse nursing students. Journal of Nursing Education, 35, 315-321.

Zeitlin-Ophir, I., Melitz, O., Miller, R., Podoshin, P., \& Mesh, G. (2004). Variables affecting the academic and social integration of nursing students. Journal of Nursing Education, 43, 326-329.

Zorn, C. R. (1996). The long-term impact on nursing students of participating in international education. Journal of Professional Nursing, 12, 106-110. 
APPENDICES 


\section{APPENDIX A}

\section{Schematic Representation of Demographic Factors Influencing Students' Cultural}

\section{Competency}

\section{Schematic Representation of Demographic Factors \\ Influencing Students' Cultural Competency}

\begin{tabular}{|c|c|}
\hline $\begin{array}{l}\text { Personal Factors } \\
\text { Age, Gender, Ethnicity/Race, Language spoken } \\
\text { at home. }\end{array}$ & $\begin{array}{c}\text { Cultural Competency by } \\
\text { Total CCA Scale Scores }\end{array}$ \\
\hline $\begin{array}{l}\text { Educational Factors } \\
\text { Program Level: BSN \& MSN } \\
\text { Program Track: Traditional BSN, RN-BSN, FEP } \\
\text { BSN; FEP BSN/MSN, MSN ARNPs (Adult, Child, } \\
\text { \& Family; \& MSN Anesthesiology } \\
\text { Program Standing (GPAs): 2.5-3.4 \& 3.5-4.0 }\end{array}$ & $\begin{array}{l}\text { Cultural Awareness and } \\
\text { Sensitivity } \\
\text { by (CAS) subscale scores } \\
\text { Culturally Competent } \\
\text { Behaviors } \\
\text { by (CCB) subscale scores }\end{array}$ \\
\hline $\begin{array}{l}\text { Social Factors } \\
\text { Ethnic/Racial Encounters; } \\
\text { Special Populations Encountered. } \\
\text { Previous diversity training: college course, } \\
\text { continuing education, online computer- } \\
\text { assisted; employer sponsored; \& professional } \\
\text { seminar/conference. }\end{array}$ & $\begin{array}{l}\text { Q5_Self-Evaluation of } \\
\text { Cultural Competency }\end{array}$ \\
\hline
\end{tabular}




\section{APPENDIX B \\ Permission to Use Instrument}

From: Stephanie Myers Schim <s.schim@wayne.edu>

Date: November 3, 2010, 3:30:27 PM EDT

To: Paula Glass <paulaglassnp@gmail.com>

Subject: Re: Cultural Competence Assessment Instrument

Dear Paula -

I am delighted to learn of your interest in the Cultural Competence Assessment instrument I developed with my team. I am sending for your review three documents:

1) an MS Word version of the most recent CCA tool

2) a couple of pages that describe how the items are scored

3) a bibliography of papers describing the tool development, use to date, etc.

Once you have a chance to review this material, I would be happy to schedule a phone conversation with you as needed. Let me know if you have any trouble downloading the documents.

We do not charge for the use of the tool at this time for students or faculty engaging in research. We do ask that you give our team credit as the source of the tool and let us know what you find if you choose to use it in your work. You may, of course, change the demographic items to suit your particular study population and research questions. Thanks again for your interest and please feel free to contact me again as needed. Best Regards -

Stephanie Myers Schim, PhD, RN, PHCNS-BC

Associate Professor

Family, Community, and Mental Health Nursing Wayne State University

240 Cohn Building

(313) 577-4034

s.schim@wayne.edu 
VITA

\section{PAULA ELLEN SEIDEL GLASS}

\section{Education}

2009-2013

Florida

2004

2004

2002-2004

$1977-1981$

\section{Employment}

$2012-2013$

2006-2012

2004-2006
Doctoral Candidate, Florida International University, Miami,

Academic Achievement Award

Florida International University, Miami, Florida

Sigma Theta Tau Induction, Nursing International Honor Society Florida International University, Miami Chapter

M.S. in Nursing, Adult Nurse Practitioner

Florida International University, Miami, Florida

B.S. in Nursing, SUNY at Buffalo, Buffalo, New York
Adjunct Faculty, Florida International University

College of Nursing and Health Sciences, Miami, Florida

Professor I - Nursing, Palm Beach State College (formerly PBCC)

Lake Worth, Florida

Adult Nurse Practitioner, Planned Parenthood, Boca Raton, Florida Scott Tannenbaum, Physiatrist - Sunrise Rehabilitation Hospital Dr. Robert Barbarite - Coral Springs, Florida

\section{Publications and Presentations}

Glass, P. (2010). Book review: Participatory development in Appalachia: Cultural identity, community, and sustainability by Keefe, S. E. (2009). Journal of Cultural Diversity, 17(4), 154-156.

Glass, P. (2011). Differences Among Undergraduate and Graduate Nursing Students' Cultural Competency Levels. Poster presentation at Florida International University Research Symposium, Miami, FL. 